\title{
Cosmic Rays from the Knee to the Highest Energies *
}

\author{
Johannes Blümer ${ }^{1}$, Ralph Engel ${ }^{1}$, and Jörg R. Hörandel ${ }^{2}$ \\ ${ }^{1}$ Karlsruhe Institute of Technology (KIT)† Institut für Kernphysik, \\ P.O. Box 3640, 76021 Karlsruhe, Germany \\ ${ }^{2}$ Radboud University Nijmegen, Department of Astrophysics, \\ P.O. Box 9010, 6500 GL Nijmegen, The Netherlands
}

April 4, 2009

\begin{abstract}
This review summarizes recent developments in the understanding of high-energy cosmic rays. It focuses on galactic and presumably extragalactic particles in the energy range from the knee $\left(10^{15} \mathrm{eV}\right)$ up to the highest energies observed $\left(>10^{20} \mathrm{eV}\right)$. Emphasis is put on observational results, their interpretation, and the global picture of cosmic rays that has emerged during the last decade.
\end{abstract}

\section{Contents}

1 Introduction $\quad 1$

2 Detection Techniques $\quad 8$

3 Energy Spectra

4 Composition $\quad[28$

5 Anisotropy

6 Astrophysical Interpretation 442

7 Importance of Modeling Hadronic Interactions

8 Conclusions and Outlook

\section{Introduction}

Cosmic rays are ionized atomic nuclei reaching the Earth from outside the Solar System. Although already discovered in 1912, their sources and propagation mechanisms are still subject of intense research. During the last decade significant progress has been made and a consistent

*Invited review, submitted to Progress in Particle and Nuclear Physics.

${ }^{\dagger} \mathrm{KIT}$ is the cooperation of University Karlsruhe and Forschungszentrum Karlsruhe 
picture of cosmic-ray observations begins to evolve. This review describes recent progress in the exploration of the sources and propagation of high-energy cosmic rays, focusing on observational results and the emerging global picture.

Many reviews of cosmic-ray theory and observations are available in the literature. Most of these reviews concentrate on different aspects and energy ranges of high-energy cosmic rays which, in turn, are presented in much more detail than possible here. For example, covering the knee energy region, experimental data are compiled in [1, 3] and a comparison of observations and model predictions can be found in [4]. The data in the range between the knee and the ankle, and their interpretation are discussed in [5]. Focusing on the upper end of the cosmicray spectrum, measurement techniques and observations are reviewed in [7, 8, 9, 13]. More theoretical and phenomenological aspects of the physics of ultra high-energy cosmic rays are subject of the reviews [14] and different source scenarios are discussed in depth in [18, 19] (acceleration scenarios) and [20] (non-acceleration scenarios). An exhaustive compilation of experimental results of the full cosmic-ray energy range can be found in [21] and a recent review, emphasizing measurement and analysis techniques, is given in [22].

In this article, we discuss high-energy cosmic-ray measurements covering the energy range from the knee to the highest energies. By concentrating mainly on observational results of the last decade and their implications for our overall understanding of cosmic rays, this review is complementary to the other articles.

The exploration of cosmic rays is mainly driven by new experimental findings. Hence, we begin this review with a short historical overview, followed by an introduction to the physics of high-energy cosmic rays (Sec.11).

In the energy region of interest cosmic rays are measured indirectly with large detector installations below the atmosphere, registering secondary particles produced in extensive air showers, initiated by high-energy cosmic rays. In Sec.2 2 basic properties of air showers are introduced and major detection techniques are discussed.

Recent experimental results concerning the flux of cosmic rays, their elemental composition, and studies of anisotropies in their arrival directions are presented in sections 3 to 5 . The global picture evolving from these measurements and their impact on the present understanding of the origin of high-energy cosmic rays is emphasized in Sec.6. The importance of the understanding of high-energy hadronic interactions for the interpretation of air shower data is underlined in Sec.7. Concluding remarks (Sec.8) complete the review.

\section{Historical Overview}

Cosmic rays were discovered in the year 1912 by V.F. Hess during several ascends with hydrogenfilled balloons up to altitudes of $5 \mathrm{~km}$ [23]. He measured the ionization rate of air as function of altitude. Electrometers served as standard devices to measure ionizing radiation at this time [24]. Hess found an increase of ionizing radiation with increasing height and he concluded that radiation penetrates from outer space into the atmosphere. For the discovery of the cosmic radiation, V.F. Hess has been awarded the Nobel Price in 1936. During the subsequent years W. Kolhörster made further ascends with improved electrometers, measuring the altitude variation of the ionization up to heights of $9 \mathrm{~km}[25$.

In $1929 \mathrm{~W}$. Bothe and W. Kolhörster measured coincident signals in two Geiger-Müller counters [26]. Placing absorber material in between the two counters they also measured the absorption characteristics of the radiation. They concluded that the "Höhenstrahlung" (or cosmic radiation) is of corpuscular nature, i.e. consists of charged particles. Similar conclusions were drawn from measurements by J. Clay, who showed that the intensity of cosmic rays depends on the (magnetic) latitude of the observer [27]. This was a clear indication that a large fraction of cosmic radiation consists of charged particles. 
Kolhörster continued his work with Geiger-Müller tubes operated in coincidence. In February 1938 he reported the discovery of coincident signals between two tubes with distances as far as $75 \mathrm{~m}$ [28]. He concluded that the tubes were hit by secondary particles or showers generated by cosmic rays in the atmosphere. In the late 1930s P. Auger undertook investigations of cosmic radiation at the Jungfraujoch, Switzerland at 3500 m.a.s.l. He used Wilson chambers and Geiger-Müller tubes separated by large distances and operated in coincidence [29]. Similar to Kolhörster, Auger concluded that the registered particles are secondaries generated in the atmosphere, originating from a single primary cosmic ray.

In the 1940s the origin of the primary radiation could be revealed with measurements on balloons at high altitudes. M. Schein showed that the positively charged primary particles were mostly protons [30]. Cloud chambers and photographic plates were carried into the stratosphere and it was found that cosmic rays are made up of fully ionized atomic nuclei moving at speeds closely to that of light [31. Many nuclei of the periodic table up to $Z \approx 40$ were found and their relative abundances determined. Hydrogen and helium occur most frequently, and the distribution in mass of the heavier nuclei appeared to be similar to that in the solar system. Elements more massive than iron or nickel were found to be very rare.

Since the mid 1940s large detector arrays were installed to measure extensive air showers. For most investigations detectors with a large surface and a short time resolution were required. Early detectors comprised Geiger-Müller counters, progress in the development of photomultipliers lead to the application of scintillation counters and the newly available Cherenkov detectors. It was found that the energy spectrum of cosmic rays follows a power law $d N / d E \propto E^{\gamma}$ over a wide range in energy. In 1958 G.V. Kulikov and G.B. Khristiansen measured the integral electron number spectrum in air showers using an array of hodoscope counters [32. They recognized a kink in the spectrum around $6 \times 10^{5}$ particles, corresponding to primary energies of several PeV $\left(10^{15} \mathrm{eV}\right)$. This structure is now known as the "knee" in the energy spectrum. Since that time there is an ongoing debate about the origin of this structure.

In the 1960s the air shower array of the M.I.T. group at Volcano Ranch, New Mexico was the largest cosmic-ray detector. The set-up comprised 20 stations equipped with scintillation counters, set up on a triangular grid, covering a total area of $12 \mathrm{~km}^{2}$. In 1962 the first event with an energy of about $10^{20} \mathrm{eV}$ has been recorded with this array [33]. Bigger air shower arrays were built (SUGAR [34, Haverah Park [35, Yakutsk [36, and AGASA [37]) and, after some initial attempts, the first successful fluorescence light detector, called Fly's Eye, was set up in Utah [38. With these detectors, another feature of the cosmic-ray flux - first discussed in [39]

- was firmly established in the early 1990s and is now known as the "ankle" [40, 42].

Finally it should be mentioned that, in the early years, particle physics was done mainly by studying cosmic rays. In the 1930s investigations of the cosmic radiation lead to the discovery of new elementary particles such as the positron [43] or the muon [44]. The pion was discovered exposing nuclear emulsions to cosmic radiation at mountain altitudes in 1947 [45]. New unstable hadrons were found in cosmic-ray interactions in balloon-borne emulsion chambers [46] in 1971 which were later, after the discovery of charm particles, identified as D mesons [47]. Also a number of exotic phenomena were observed [48], none of which could be confirmed in accelerator experiments.

Over time a standard description of cosmic rays evolved, which is briefly sketched in the following section.

\section{Nature and Origin of Cosmic Rays}

The solar system is permanently exposed to a flux of highly energetic ionized atomic nuclei the cosmic rays. Their energies extend from the $\mathrm{MeV}$ range to at least $10^{20} \mathrm{eV}$. The differential energy spectrum of all cosmic-ray particles is depicted in Fig.1. It falls steeply as function of 


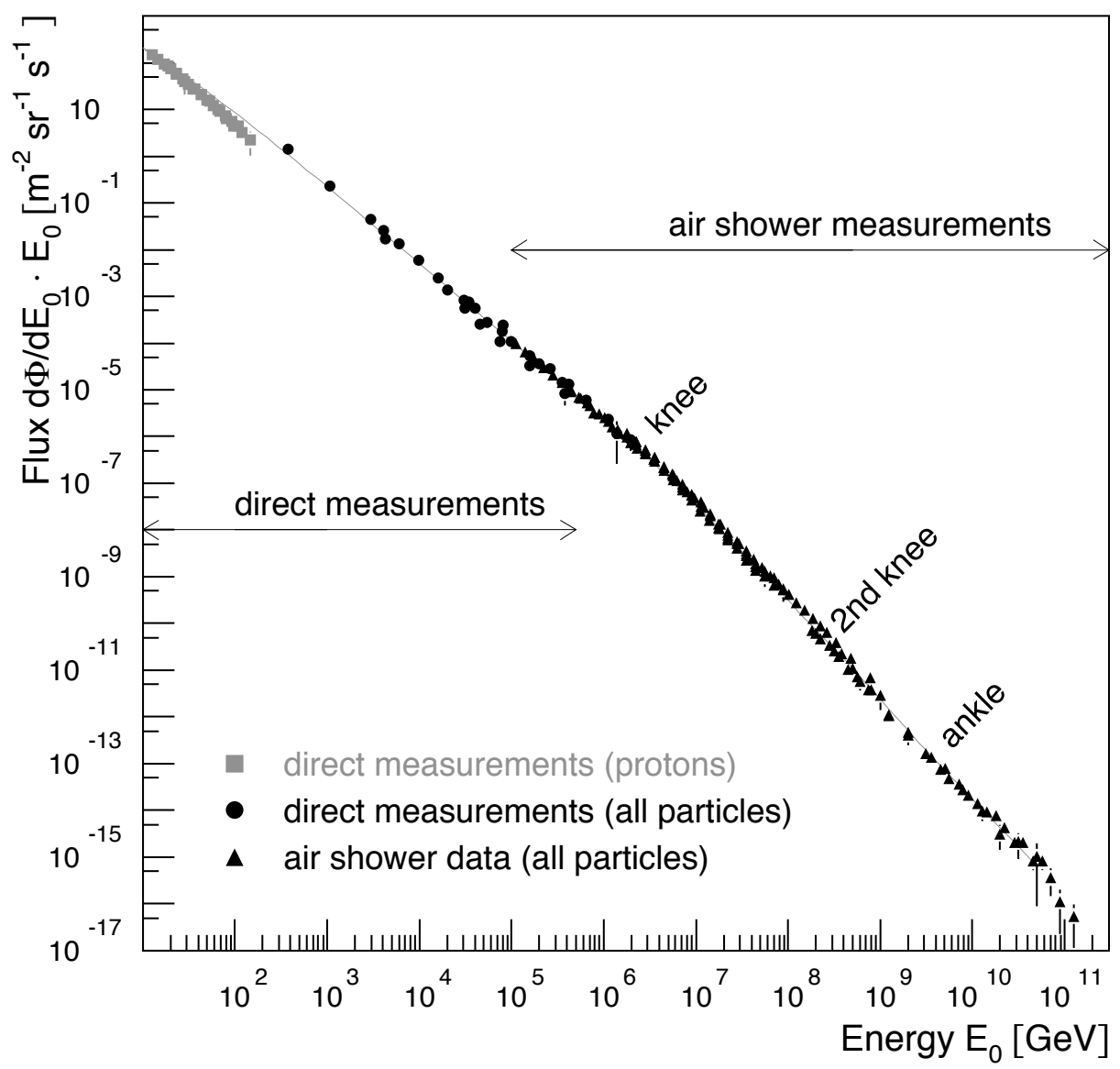

Figure 1: All-particle energy spectrum of cosmic rays as measured directly with detectors above the atmosphere and with air shower detectors. At low energies, the flux of primary protons is shown.

energy, decreasing by about a factor 500 per decade in energy. The flux decreases from more than 1000 particles per second and square meter at $\mathrm{GeV}$ energies to about one particle per $\mathrm{m}^{2}$ and year at a $\mathrm{PeV}$, and further to less than one particle per $\mathrm{km}^{2}$ and century above $100 \mathrm{EeV}$.

The strong decrease in flux poses a big experimental challenge and our knowledge about the particles and their origin is more and more limited with increasing energy (and decreasing flux). At sub-GeV energies individual isotopes are measured with small detectors in outer space and individual elements can be resolved with balloon-borne detectors in the $\mathrm{TeV}$ regime. At energies exceeding $100 \mathrm{TeV}$ large detection areas are required to collect a suitable number of particles in a reasonable time. At present, such detectors are realized at ground level only and secondary particles generated in the atmosphere (the extensive air showers) are registered. At $\mathrm{PeV}$ energies, groups of elements could be resolved, while at the highest energies even a classification into "light" and "heavy" particles becomes already an experimental challenge.

The energy spectrum follows a power law $d N / d E \propto E^{\gamma}$ over a wide energy range, indicating non-thermal acceleration processes. The spectrum is rather featureless as can be inferred from Fig.1. However, small structures become clearly visible when the ordinate is multiplied with some power of the particle energy, as shall be discussed below (see e.g. Fig.7). The spectral index is $\gamma \approx-2.7$ at energies up to several $\mathrm{PeV}$. Then a steepening is observed, the so-called knee, with $\gamma \approx-3.1$ at higher energies. A further steepening, the second knee, occurs around 


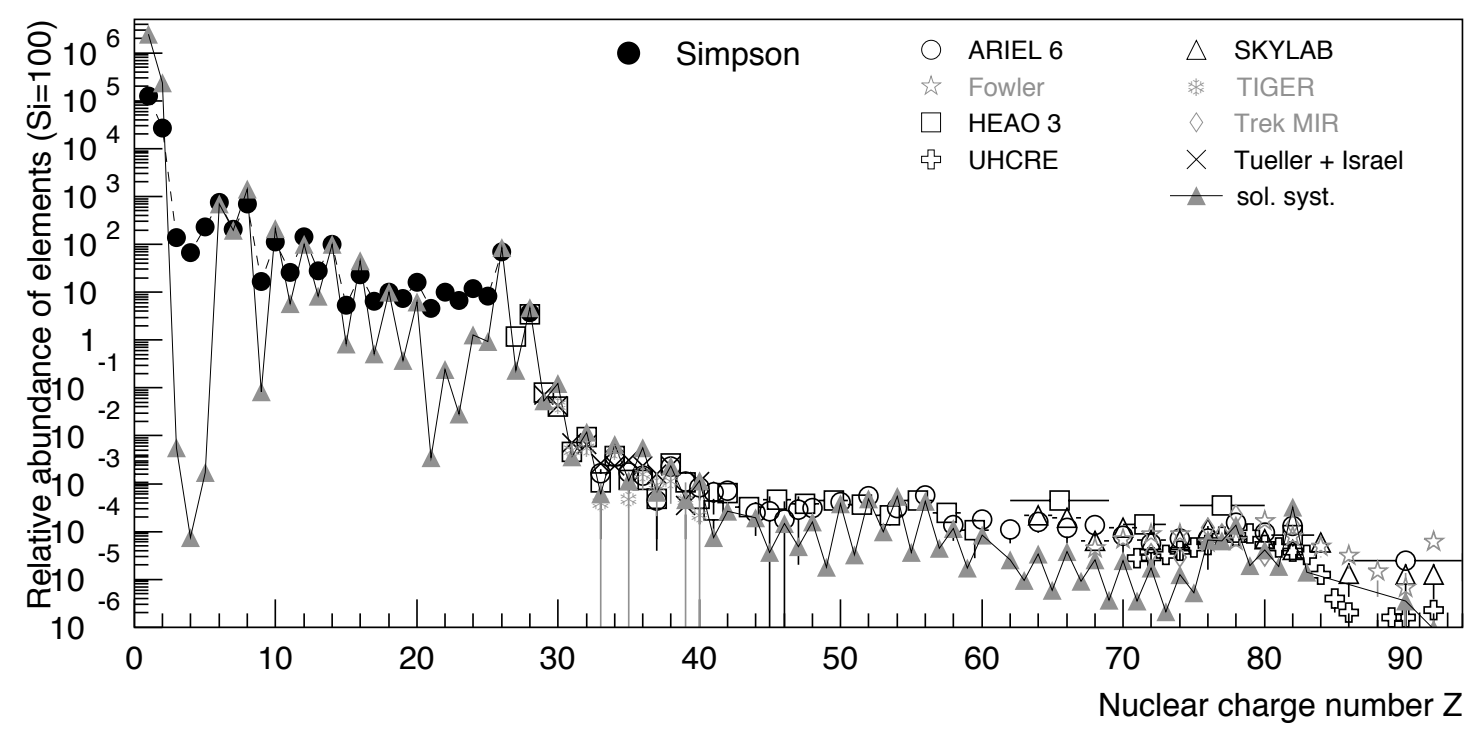

Figure 2: Abundance of elements in cosmic rays as function of their nuclear charge number $Z$ at energies around $1 \mathrm{GeV} / \mathrm{n}$, normalized to $S i=100$ [50]. Abundance for nuclei with $Z \leq 28$ according to [51]. Heavy nuclei as measured by ARIEL 6 [52], [53], HEAO 3 [54], SKYLAB [55], TIGER [56], TREK/MIR [57], [58], as well as UHCRE [59]. In addition, the abundance of elements in the solar system is shown according to 60].

$4 \times 10^{17} \mathrm{eV}$. Finally, at about $4 \times 10^{18} \mathrm{eV}$, at the ankle, the spectrum flattens again.

The abundance of elements in cosmic rays is shown in Fig. 1 as function of the nuclear charge number. All elements of the periodic table have been found in cosmic rays. For the relatively abundant elements up to nickel, energy spectra for individual elements have been measured [1, 3]. Abundances as obtained by several experiments at about $1 \mathrm{GeV} / \mathrm{n}$ are depicted. The cosmicray composition is compared to the abundance of elements in the solar system. Overall, both distributions look very alike. However, there exist certain differences, which reveal information on the acceleration and propagation of cosmic rays.

The light elements lithium, beryllium, and boron as well as the elements below iron $(Z=26)$ and below lead $(Z=82)$ are more abundant in cosmic rays than in the solar system. They are assumed to be produced in spallation processes of the more abundant particles of the CNO, iron, and lead groups during the journey of cosmic rays through the Galaxy. Hence, they are frequently referred to as secondary cosmic rays. As the spallation cross section of the relevant nuclei is known at $\mathrm{GeV}$ energies, the ratio of secondary to primary cosmic rays is used to infer the propagation path length of cosmic rays in the Galaxy. An example is the boron-to-carbon ratio which has been measured as function of energy 61. The ratio decreases as function of energy which is frequently explained in Leaky Box models by a rigidity-dependent 11 decrease of the path length of cosmic rays in the Galaxy $\Lambda(R)=\Lambda_{0}\left(R / R_{0}\right)^{-\delta}$. Typical values are $\Lambda_{0} \approx 10-15 \mathrm{~g} / \mathrm{cm}^{2}$, $\delta \approx 0.5-0.6$, and $R_{0} \approx 4 \mathrm{GV}$ as reference rigidity.

Cosmic-ray particles are assumed to propagate in a diffusive process through the Galaxy, being deflected many times by the randomly oriented magnetic fields ( $B \sim 3 \mu \mathrm{G}$ ). The nuclei are not confined to the galactic disc, they propagate in the galactic halo as well. The scale height of the halo has been estimated with measurements of the ${ }^{10} \mathrm{Be} /{ }^{9} \mathrm{Be}$-ratio by the ISOMAX detector 62 to be a few kpc. The abundance of radioactive nuclei in cosmic rays measured with the CRIS instrument yields a residence time in the Galaxy of about $15 \times 10^{6}$ years for particles with

\footnotetext{
${ }^{1}$ Rigidity is defined as particle momentum divided by its charge $R[\mathrm{~V}]=p / z$.
} 
GeV energies 63].

The energy density of cosmic rays amounts to about $\rho_{c r} \approx 1 \mathrm{eV} / \mathrm{cm}^{3}$, a value comparable to the energy density of the visible star light $\rho_{s l} \approx 0.3 \mathrm{eV} / \mathrm{cm}^{3}$, the galactic magnetic fields $B^{2} / 2 \mu_{0} \approx 0.25 \mathrm{eV} / \mathrm{cm}^{3}$, or the microwave background $\rho_{3 K} \approx 0.25 \mathrm{eV} / \mathrm{cm}^{3}$. The power required to sustain a constant cosmic-ray intensity can be estimated as $L_{c r}=\rho_{c r} V / \tau_{\text {esc }} \approx 10^{41} \mathrm{erg} / \mathrm{s}$, where $\tau_{\text {esc }}$ is the residence time of cosmic rays in a volume $V$ (the Galaxy and the galactic halo). With a rate of about three supernovae per century in a typical Galaxy, the energy required could be provided by a small fraction $(\approx 10 \%)$ of the kinetic energy released in supernovae. This had been realized already in 1934 by Baade and Zwicky [64]. The actual mechanism of acceleration remained mysterious until Fermi [65] proposed a process that involved interaction of particles with large-scale magnetic fields in the Galaxy. Eventually, this lead to the currently accepted model of cosmic-ray acceleration by the first-order Fermi mechanism that operates in strong shock fronts which are powered by supernova explosions and propagate from a supernova remnant (SNR) into the interstellar medium [66].

Diffusive, first-order shock acceleration works by virtue of the fact that particles gain an amount of energy $\Delta E \propto E$ at each cycle, where a cycle consists of a particle passing from the upstream (unshocked) region to the downstream region and back. At each cycle, there is a probability that the particle is lost downstream and does not return to the shock. Higher energy particles are those that have remained longer in the vicinity of the shock and so have had time to achieve higher energy. After a time $T$ the maximum energy achieved is $E_{\max } \sim Z e \beta_{s} \cdot B \cdot T V_{s}$, where $\beta_{s}=V_{s} / c$ refers to the velocity of the shock. This results in an upper limit, assuming a minimal diffusion length equal to the Larmor radius of a particle of charge $Z e$ in the magnetic fields $B$ behind and ahead of the shock. Using typical values of Type II supernovae exploding in an average interstellar medium yields $E_{\max } \approx Z \cdot 10^{14} \mathrm{eV}$ [71]. More recent estimates give a maximum energy up to one order of magnitude larger for some types of supernovae [72]. It has also been suggested that the cosmic rays itself interact with the magnetic fields in the acceleration region, yielding to an amplification of the fields, which in turn results in much higher energies that can be reached during the acceleration process [73]. With this mechanism cosmic rays are supposedly accelerated up to $10^{17} \mathrm{eV}$.

Information on the composition at the source can be obtained from measurements of the abundance of refractory nuclei. They appear to have undergone minimal elemental fractionation relative to one another. Comparing the derived abundance at the source to the abundance in the solar system reveals that the two samples exhibit a striking similarity over a wide range [74]. When uncertainties are taken into account, the abundances of particular isotopes are consistent with being within $20 \%$ of the solar values. This indicates that cosmic rays are accelerated out of a sample of well mixed interstellar matter. Hence, cosmic rays are "regular" matter, but accelerated to extremely high energies.

The understanding of the origin of the knee in the energy spectrum is commonly thought to be a cornerstone for the understanding of the origin of (galactic) cosmic rays. Many approaches are discussed in the literature [4. A popular explanation is that the knee is associated with the upper limit of acceleration by galactic supernovae, while the ankle is associated with the onset of an extragalactic population that is less intense but has a harder spectrum that dominates at sufficiently high energy. Another popular explanation is leakage of particles from the Galaxy. At energies in the $\mathrm{GeV}$ regime measurements indicate a decreasing path length of cosmic rays in the Galaxy. Extrapolating this to higher energies indicates that above a certain energy cosmic rays are not contained in the Galaxy (or the galactic halo) anymore. In a simple picture, this can be understood since the Larmor radius of a proton in the galactic magnetic field

$$
r_{L}=1.08 \mathrm{pc} \frac{E / \mathrm{PeV}}{Z \cdot B / \mu \mathrm{G}}
$$



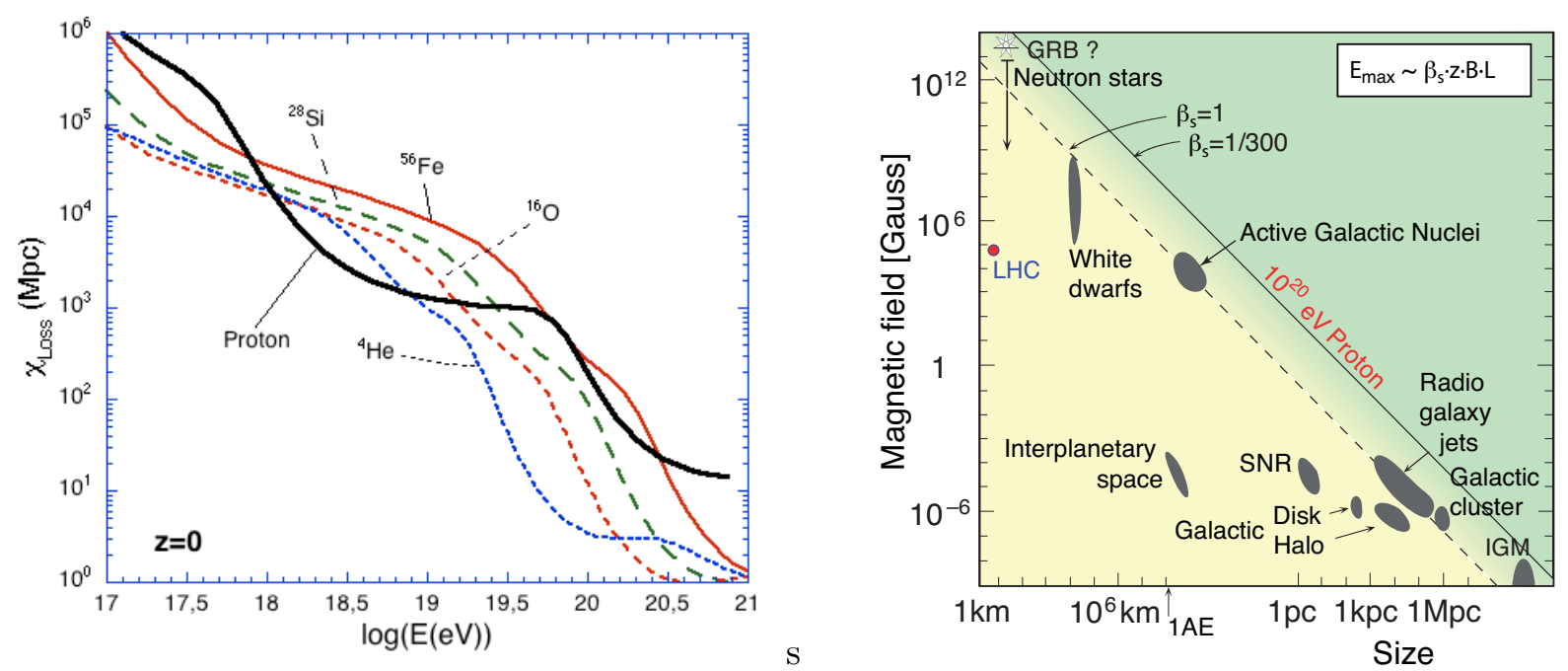

Figure 3: Left panel: Energy loss lengths of protons and nuclei calculated for a redshift $z=0$ [86]. Right panel: Hillas plot [18] of astrophysical objects in which cosmic rays could be accelerated (adapted from [88]).

becomes with increasing energy comparable to and finally exceeds the thickness of the galactic disk.

If the knee is caused by the maximum energy attained during the acceleration process or due to leakage from the Galaxy the energy spectra for individual elements with charge $Z$ would exhibit a cut-off (or a knee) at an energy $E_{c}^{Z}=Z \cdot E_{c}^{p}$, with the cut-off energy $E_{c}^{p}$ for protons. The sum of the flux of all elements with their individual cut-offs makes up the all-particle spectrum. In this picture the knee in the all-particle spectrum is related to the cut-off for protons and the steeper spectrum above the knee is a consequence of the subsequent cut-offs for all elements, resulting in a relatively smooth spectrum above the knee. ${ }^{2}$ Since the abundance of ultra-heavy nuclei (see Fig. 1) at GeV energies is very low as compared to iron the end of the galactic component is often assumed to be at energies around $30 \times E_{c}^{p}$. However, recently it has been proposed that ultra-heavy elements may play an important role at high energies [3] which yields a value of $92 \times E_{c}^{p}$ for the end of the galactic component, coinciding with the second knee at $4 \times 10^{17} \mathrm{eV}$.

Another interesting question is that of a natural end of the spectrum at high energy. Already 40 years ago it has been realized that interactions of cosmic rays with photons of the cosmic microwave background (CMB) would result in a cut-off of the spectrum above $6 \times 10^{19} \mathrm{eV}$ [77]. All hadronic particles suffer significant energy losses during propagation above this energy. Protons interact with background photons forming mainly a $\Delta^{+}(1232)$ resonance [79] and nuclei are broken up due to photodisintegration [83]. A compilation of energy loss lengths of protons and various nuclei is shown in Fig. 3 (left) [86]. The energy loss length of photons depends on the flux of the universal radio background (URB) which is not well known (see, for example, discussion in [87]). Depending on the assumptions on the URB, the energy loss length is significantly smaller than or comparable to that of hadronic particles.

The short energy loss lengths indicate that cosmic rays with energies above $10^{20} \mathrm{eV}$ should come from sources within $\mathrm{a} \sim 100 \mathrm{Mpc}$ sphere. Astrophysical sources within our Galaxy are disfavored. Even though rapidly spinning young neutron stars could be thought of accelerating particles to the highest energies observed [89], it would be difficult to explain the apparent

\footnotetext{
${ }^{2}$ Such a scenario has been pointed out first by Peters 75 and it has been suggested to call such a behavior a Peters cycle [76].
} 
isotropic arrival direction of UHECRs to energies beyond $10^{19} \mathrm{eV}$. As cosmic rays of energy greater than $10^{18} \mathrm{eV}$ are no longer confined by galactic magnetic fields, it is natural to assume that they are produced by extragalactic sources.

Considering diffusive shock acceleration, which is thought to accelerate cosmic rays in SNRs, the magnetic field strength $B$ in the source and the size $R$ of the source region are related to the maximum acceleration energy by [18]

$$
E_{\max } \simeq 10^{18} \mathrm{eV} Z \beta_{s}\left(\frac{R}{\mathrm{kpc}}\right)\left(\frac{B}{\mu \mathrm{G}}\right)
$$

where $\beta_{s}$ is the shock velocity in units of $c$ and $Z$ is the particle charge. This relation is shown in Fig. 3 (right) for various astrophysical objects. The list of the very few viable candidate sources includes active galactic nuclei (AGN) [90, 91, 92, radio lobes of FR II galaxies [93, 94, and gamma-ray bursts (GRBs) [95] (for a review of astrophysical sources, see [19]).

Many alternative, non-acceleration scenarios - called top-down models - have been proposed. In these models, UHECRs are produced in decays of super-heavy objects such as super-heavy dark matter [98], cryptons [100], or topological defects [101]. All of these models postulate new particle physics and predict typically high gamma-ray fluxes at ultra-high energy [102]. Finally there are propagation model scenarios in which the GZK energy loss processes are evaded or shifted to higher energies. Examples are violation of Lorentz invariance [105], the $Z$-burst model [110] or postulation of new particles with properties similar to protons [113, 114]. Reviews of the different scenarios can be found in [20, 115].

Measurements of the arrival direction distribution, primary mass composition and flux will be the key ingredients to solving the puzzle of UHECR sources. Given an expected angular deflection of only a few degrees for particles above $10^{19.5} \mathrm{eV}$ in our Galaxy [116] and the existence of large cosmic voids with negligible magnetic fields [119], high statistics measurements should finally allow cosmic-ray astronomy and reveal correlations with sources or source regions. Similarly, knowing the composition of UHECRs will restrict the classes of source models. For example, a mixed composition would exclude top-down models. Another very important source of complementary information is given by secondary particle fluxes, i.e. gammarays and neutrinos, produced in UHECRs sources and during propagation (see, for example, [122, 123, 124, 86, 125, 126]).

\section{Detection Techniques}

\section{Shower Properties}

In the following we will introduce some general properties of extensive air showers that are employed in cosmic-ray measurements. Detailed presentations of this subject can be found in [127.

\section{Electromagnetic Showers}

Showers initiated by photons or electrons (called em. showers henceforth) are governed mainly by the particle production processes (i) bremsstrahlung of electrons ${ }^{3}$ and (ii) pair production of electrons by photons. In addition to radiative energy losses, electrons are subject to ionization energy loss. The total energy loss $d E / d X$ of electrons can be written as

$$
\frac{d E}{d X}=-\alpha(E)-\frac{E}{X_{R}}
$$

\footnotetext{
${ }^{3}$ In the following we will use the term electron to refer to both electrons and positrons.
} 
where $\alpha(E)$ is the ionization energy loss given by the Bethe-Bloch formula which depends logarithmically on energy. The radiation length, $X_{R}$, depends on the material the shower evolves in and is $X_{R} \approx 37 \mathrm{~g} / \mathrm{cm}^{2}$ in air. Particle multiplication and ionization energy loss are competing processes in showers. The energy at which an electron loses the same energy due to ionization and particle production is called critical energy, $E_{c}=X_{R}\left\langle\alpha\left(E_{c}\right)\right\rangle \approx 86 \mathrm{MeV}$.

Some properties of em. showers can already be understood within the very simple model of Heitler [130]. In this model it is assumed that the incoming particle interacts in the atmosphere after traveling a depth distance $\lambda_{\mathrm{em}}$ and produces two new particles with half the energy of the initial particle. These two new particles again interact at a distance $\lambda_{\mathrm{em}}$ from their production point. After $n$ generations of successive interactions the number of particles is $2^{n}$. The number of particles as function of depth $X$ can be written as $N(X)=2^{X / \lambda_{\mathrm{em}}}$. The production of new particles continues until the energy of the secondary particles is smaller than the critical energy $E_{c}$, below which absorption processes dominate over further particle multiplication. The maximum number of particles in the shower is $N_{\max }=E_{0} / E_{c}$ and the depth of shower maximum is given by $X_{\max }=\lambda_{\mathrm{em}} \log _{2}\left(E_{0} / E_{c}\right)$, with $E_{0}$ being the primary particle energy. Heitler's model is certainly an over-simplified picture of an air shower but illustrates two important features. The number of particles at shower maximum is approximately proportional to the primary energy and the depth of shower maximum increases logarithmically with energy.

Approximate formulae for the longitudinal shower size profile and the lateral particle distribution of em. showers have been derived from cascade theory [131, 132]. Considering only shower particles of energy $E$, the depth of shower maximum is given by $X_{\max } \approx X_{R} \ln \left(E_{0} / E\right)$. Accounting for the energy distribution of particles in a shower this expression becomes (here written for electrons in a photon-induced shower)

$$
X_{\max } \approx X_{R}\left[\ln \left(\frac{E_{0}}{E_{c}}\right)+\frac{1}{2}\right]
$$

The increase of the depth of shower maximum per decade in energy is called elongation rate, $D_{10}$. The elongation rate of em. showers is energy-independent, $D_{10}^{\mathrm{em}}=\ln (10) X_{R} \approx 85 \mathrm{~g} / \mathrm{cm}^{2}$. For energies larger than $E_{c}$, the energy spectrum of secondary particles in a shower follows approximately a power law $d N / d E \sim E^{-(1+s)}$ with where $s$ denotes the shower age parameter. The shower age is often phenomenologically defined as $s \approx 3 X /\left(X+2 X_{\max }\right)$. Based on the solutions of the one-dimensional cascade equations given in [131, Greisen developed a compact and still often used parametrization of the mean longitudinal shower size profile (number of charged particles) 134

$$
N_{e}(X)=\frac{0.31}{\sqrt{\ln E_{0} / E_{c}}} \exp \left\{\left(1-\frac{3}{2} \ln s\right) \frac{X}{X_{R}}\right\} .
$$

A recent derivation of this expression is given in [135. In data analysis, a function proposed by Gaisser and Hillas [136] is often used to fit measured shower profiles

$$
N(X)=N_{\max }\left(\frac{X-X_{0}}{X_{\max }-X_{0}}\right)^{\left(X_{\max }-X\right) / \Lambda} \exp \left(\frac{X_{\max }-X}{\Lambda}\right) .
$$

The particle density in dependence on the distance to the shower core (lateral distance) is mainly determined by multiple Coulomb scattering of electrons. For electrons of low energy $E$, the increase of variance of the effective scattering angle per traversed depth $\delta X$ is approximately given by $\left\langle\delta \theta^{2}\right\rangle \approx\left(E_{s} / E\right)^{2} / X_{R} \delta X$, with $E_{s} \approx 21 \mathrm{MeV}$ [137]. Results of detailed calculations of the lateral particle distribution by Nishimura and Kamata [132] were parametrized by Greisen [138] as

$$
\frac{d N_{e}}{r d r d \varphi}=C(s) N_{e}(X)\left(\frac{r}{r_{1}}\right)^{s-2}\left(1+\frac{r}{r_{1}}\right)^{s-4.5}
$$


with $C(s)=\Gamma(4.5-s) /\left[2 \pi r_{1}^{2} \Gamma(s) \Gamma(4.5-2 s)\right]$ being a normalization constant. Eq. (7) is called Nishimura-Kamata-Greisen (NKG) function. The lateral distribution of the shower particles scales with the Molière unit $r_{1}=X_{R} E_{s} / E_{c} \approx 9.3 \mathrm{~g} / \mathrm{cm}^{2}$ and hence depends on the local air density. The effect of varying atmospheric density along the shower track can approximately be taken into account by calculating the Molière unit not at observation hight but 2-3 radiation lengths higher up in the atmosphere [134].

At very high energy, two additional processes become important and change the characteristics of em. showers. First of all, the amplitudes of subsequent interactions of photons or electrons, which are independent at low energy, have to be added coherently [139] as the interaction length becomes comparable to the separation of subsequent interactions. The resulting effect is known as Landau-Pomeranchuk-Migdal (LPM) effect and leads to the suppression of new particle production in certain kinematic regions [141. In air, the LPM effect typically becomes important at energies above $10^{18} \mathrm{eV}$ : Shower-to-shower fluctuations of em. showers increase drastically and the depth of maximum is shifted deeper into the atmosphere.

Another important ultra high-energy process is that of magnetic pair production and bremsstrahlung [143]. Gamma-rays of energies exceeding $10^{19.5} \mathrm{eV}$ can interact with the geomagnetic field of the Earth. Such interactions typically take place a thousand kilometers above the atmosphere. Mainly due to magnetic bremsstrahlung, a shower of more than 100 secondary photons and a few electrons is formed, which interact in the atmosphere simultaneously. Simulations of this effect can be found in [145, 146, 147, 148]. As the primary energy is shared by many secondary particles, the LPM effect hardly influences such showers. Due to the superposition of many lower-energy em. showers, shower-to-shower fluctuations of converted primary photons are significantly reduced. The dependence of the geomagnetic pre-shower effect on the local arrival direction can be used to search for photons in a model-independent way, see [145, 148].

\section{Hadron-initiated Showers}

Most of the differences between photon and hadron-initiated showers are related to the fact that hadronic showers develop a significant muon component whereas there are very few muons in purely em. showers. Furthermore, hadronic multiparticle production is characterized by large event-to-event fluctuations.

Again a model similar to that from Heitler is useful to understand the basic properties of hadronic showers (see, for example, [150, 151, 152, 153]). For simplicity we assume that a hadronic interaction of a particle with energy $E$ produces $n_{\text {tot }}$ new particles with energy $E / n_{\text {tot }}$, two thirds of which being charged particles $n_{\mathrm{ch}}$ (charged pions) and one third being neutral particles $n_{\text {neut }}$ (neutral pions). Neutral particles decay immediately into em. particles $\left(\pi^{0} \rightarrow 2 \gamma\right)$. Charged particles re-interact with air nuclei if their energy is greater than some typical decay energy $E_{\mathrm{dec}}$ or decay otherwise. The number of generations of hadronic interactions, $n$, follows from $E_{\mathrm{dec}}=E_{0} /\left(n_{\mathrm{tot}}\right)^{n}$.

Supposing that one muon is produced in the decay of each charged particle, one gets

$$
N_{\mu}=\left(n_{\mathrm{ch}}\right)^{n}=\left(\frac{E_{0}}{E_{\mathrm{dec}}}\right)^{\alpha},
$$

with $\alpha=\ln n_{\mathrm{ch}} / \ln n_{\text {tot }} \approx 0.86 \ldots 0.93$. The numerical values for $\alpha$ and $E_{\text {dec }}$ depend on the muon energy threshold and are given in [154] for different hadronic interaction models. The number of muons produced in an air shower increases almost linearly with primary energy and depends on the air density (through $E_{\mathrm{dec}}$ ) and the charged and total particle multiplicities of hadronic interactions.

Also the energy transferred to the em. shower component can be estimated within this simple model. In each hadronic interaction, a certain fraction of the initial energy is transferred to the 
em. shower component. After $n$ generations the energy in the hadronic and em. components is given by

$$
E_{\text {had }}=\left(\frac{2}{3}\right)^{n} E_{0}, \quad \quad E_{\text {em }}=E_{0}-E_{\text {had }} .
$$

Simulations show that the number of generations of charged pions is typically about 5 to 6 [155] and increases slightly with primary shower energy. Correspondingly the fraction of energy transferred to the em. component increases from about $70-80 \%$ at $10^{15} \mathrm{eV}$ to $90-95 \%$ at $10^{20} \mathrm{eV}$.

The depth of shower maximum of a hadron-induced shower is given by that of the em. shower component, $X_{\max }^{e}$. The first hadronic interaction produces em. particles of energy $\sim E_{0} / n_{\text {tot }}$. Therefore one can write in lowest order approximation

$$
\begin{aligned}
X_{\max }\left(E_{0}\right) & \sim \lambda_{\text {had }}+X_{\text {max }}^{e}\left(E_{0} / n_{\text {tot }}\right) \\
& \sim \lambda_{\text {had }}+X_{R} \cdot \ln \left(\frac{E_{0}}{n_{\text {tot }} E_{c}}\right),
\end{aligned}
$$

where $\lambda_{\text {had }}$ is the hadronic interaction length. From Eq. 10 follows the elongation rate theorem, stating that hadronic showers have always an elongation rate equal to or smaller than that of em. showers [156]

$$
\begin{aligned}
D_{10}^{h} & \approx\left(1-B_{\lambda}-B_{n}\right) \ln (10) X_{R} \ln \left(E_{0} / E_{c}\right) \\
& \approx\left(1-B_{\lambda}-B_{n}\right) D_{10}^{\mathrm{em}}
\end{aligned}
$$

The coefficients are $B_{\lambda}=-d \lambda_{\text {had }} / d \ln E$ and $B_{n}=d \ln \left(n_{\text {tot }}\right) / d \ln E$ (see also discussion in [154] for the next higher order term).

If the primary particle is a nucleus, one can use the superposition model to deduce the main shower characteristics [157]. In this model, a nucleus with mass $A$ and energy $E_{0}$ is considered as $A$ independent nucleons with energy $E_{\mathrm{h}}=E_{0} / A$. The superposition of the individual nucleon showers yields

$$
\begin{aligned}
N_{\max }^{A} & \approx A \cdot \frac{E_{\mathrm{h}}}{E_{c}}=\frac{E_{0}}{E_{c}}=N_{\max } \\
X_{\max }^{A} & \approx X_{\max }\left(E_{0} / A\right) \\
N_{\mu}^{A} & \approx A \cdot\left(\frac{E_{0} / A}{E_{\mathrm{dec}}}\right)^{\alpha}=A^{1-\alpha} \cdot N_{\mu} .
\end{aligned}
$$

To a good approximation, while the number of charged particles at shower maximum is independent of the primary hadron, both, the number of muons and the depth of maximum do depend on the mass of the primary particle. The heavier the shower-initiating particle the more muons are expected for a given primary energy. In addition, the superposition of $A$ independent showers naturally explains why the shower-to-shower fluctuations are smaller for shower initiated by nuclei as compared to proton showers.

Detailed shower simulations confirm the basic shower properties discussed so far. Quantitative predictions depend on the details of modeling particle production and transport [158, 159]. Whereas em. interactions are rather well understood within perturbative QED, hadronic multiparticle production cannot be calculated within QCD from first principles. Phenomenological models have to be used to describe the final states of hadronic interactions. The parameters of these models are determined by comparing the model predictions with accelerator measurements. Reflecting the different methods for describing data, low- and high-energy interaction models are distinguished. The former ones are typically based on the picture of intermediate resonance formation and decay as well as parametrizations of data. The latter ones are involving the production of color strings and their fragmentation. 

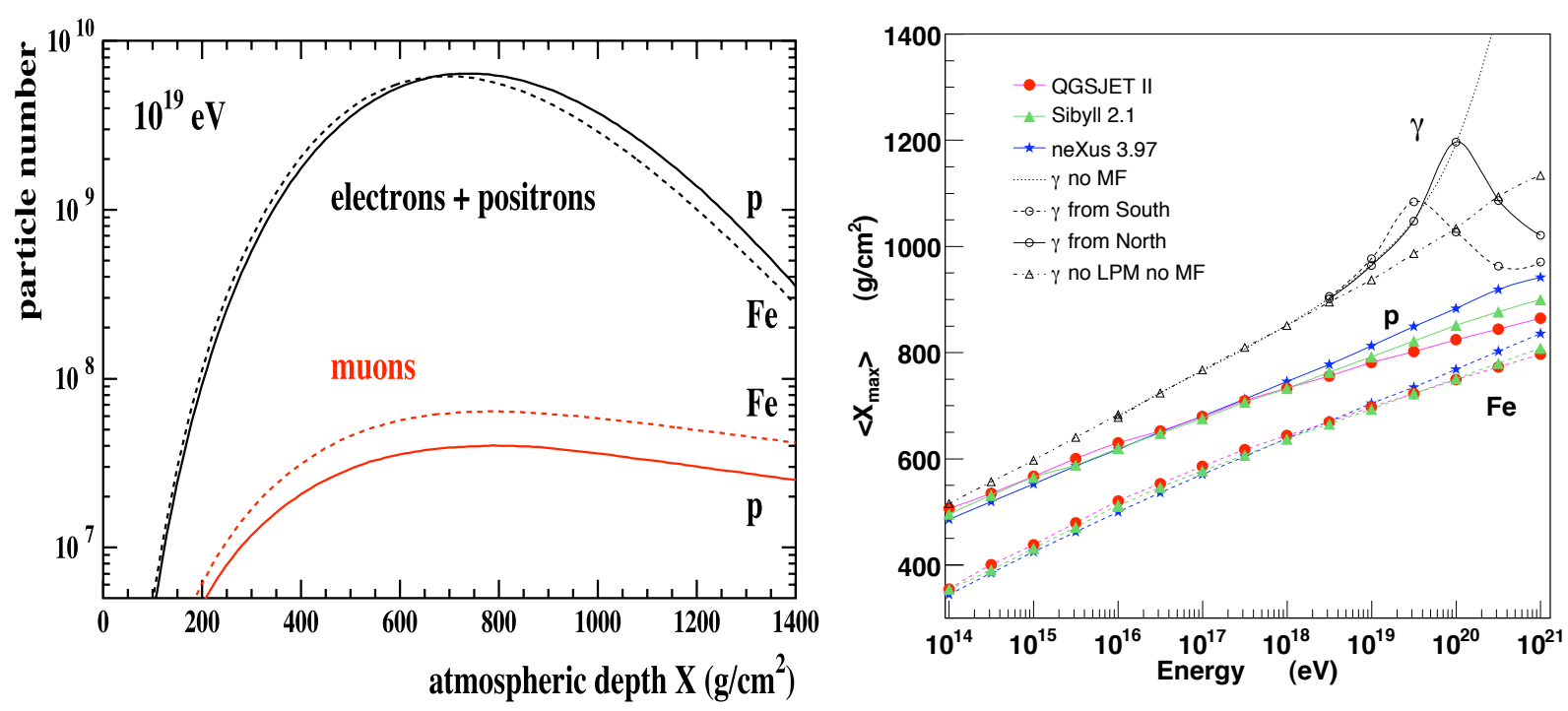

Figure 4: Left panel: Longitudinal shower profile. Shown are the number of electrons with $E_{\text {kin }}>250 \mathrm{keV}$ and muons with $E_{\text {kin }}>250 \mathrm{MeV}$ (from [151]). Right panel: Mean depth of shower maximum calculated with different hadronic interaction models and CONEX (from [184, modified).

For high energy interactions $\left(E_{\text {lab }} \gtrsim 100 \mathrm{GeV}\right)$, the hadronic interaction models often used in air shower simulations are DPMJET II.55 and III [160, 161, EPOS [162, 163], QGSJET 01 [164] and QGSJET II [167], as well as SIBYLL 2.1 [157, 169, 170]. These models reproduce accelerator data reasonably well but are characterized by different extrapolations above a center-of-mass energy $E_{\mathrm{cms}} \sim 1.8 \mathrm{TeV}\left(E \sim 10^{15} \mathrm{eV}\right)$, leading to very different shower predictions at high energy [158, 159, 171, 172.

The situation is different at low energy where more measurements from fixed target experiments are available. There, one of the main problems is the extrapolation of measurements to the very forward phase space region close to the beam direction and the lack of measurements of pion-induced interactions with light nuclei [155]. At low energy, models based on data parameterizations and/or microscopic models such as FLUKA [173], GHEISHA [174], UrQMD [175], or TARGET [176] are used. Differences of the predictions of these models are important for the number of muons [177], in particular at large lateral distances.

In Figs. 445, we illustrate some shower properties discussed only qualitatively so far by showing the results of detailed Monte Carlo simulations done with CORSIKA [182] and CONEX 183 .

The longitudinal profile of a typical proton shower of $10^{19} \mathrm{eV}$ is shown in Fig. 4 (left). In the region of the shower maximum, less than $1 \%$ of the charged shower particles are muons. The electromagnetic component is absorbed much faster than the muonic one.

As muons are mainly produced in hadronic interactions, their number and lateral distribution can be used as composition-sensitive observables. The predicted muon distributions depend on the assumptions on hadron production in air showers. This is demonstrated in Fig. 5 where the expected number of muons and electrons is shown for showers initiated by proton, iron, and gamma-ray primaries, as calculated with different interaction models [172]. The energy dependence of the total muon number and ratio between the muon numbers of proton and iron showers is in agreement with the expectations from the superposition model. At high energy, the discrimination power of electron-muon number measurements is subject to large systematic uncertainties due to our limited understanding of hadronic multiparticle production.

In Fig. 4 (right), we show the mean depth of shower maximum, $\left\langle X_{\max }\right\rangle$, for different primary 


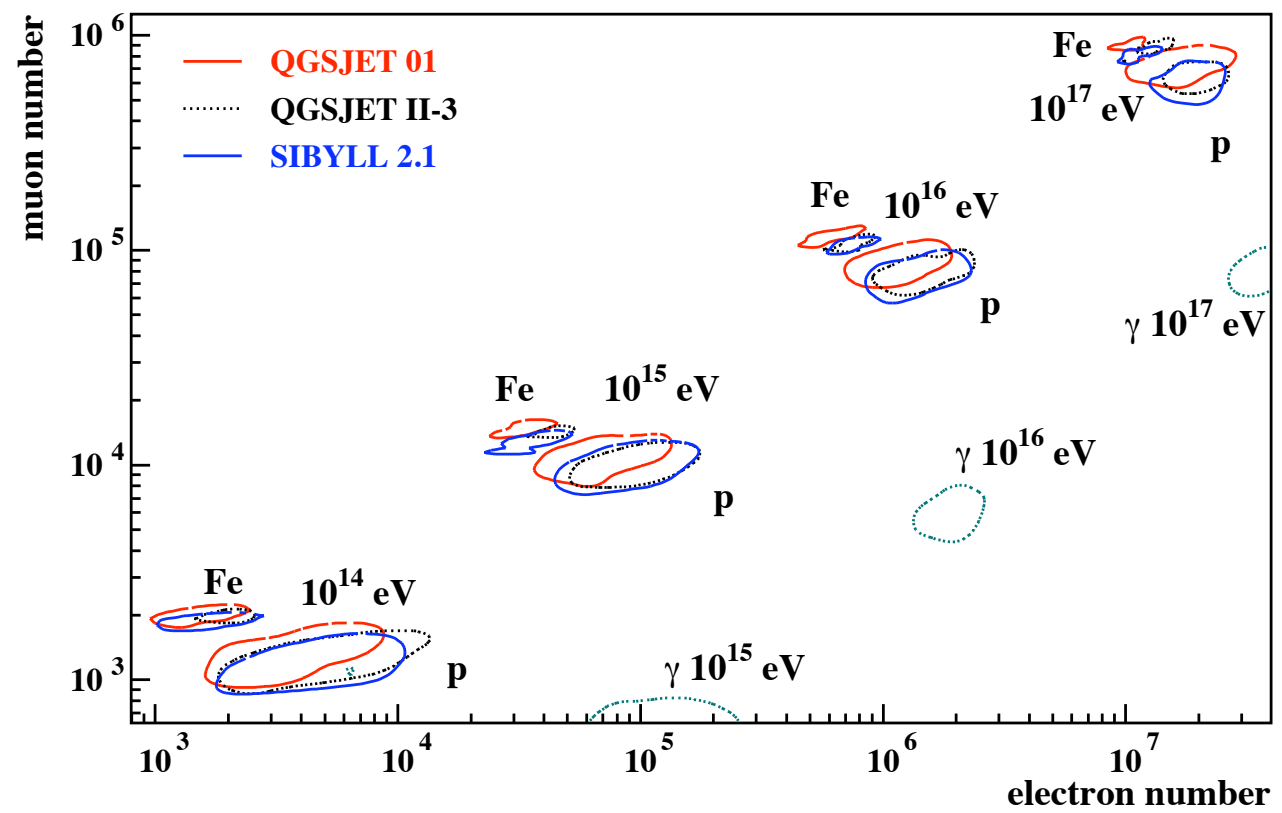

Figure 5: Expected number of muons and electrons in vertical showers at sea level. The curves show the FWHM of the distributions for different primary particles and energies, as obtained with QGSJET 01, QGSJET-II, and SIBYLL 2.1 [172, 185].

particles. The difference between proton and iron showers is in agreement with the predictions of the superposition model. Whereas the expected mean depth of maximum depends again sensitively on the chosen hadronic interaction model, the fluctuations are rather model-independent at a given energy. The elongation rate of em. showers obtained with detailed model simulations coincides with that predicted in em. cascade theory [131]. The increase of the em. elongation rate at high energy is caused by the LPM effect. Also, at ultra high-energy, photon interaction with the geomagnetic field can lead to a negative elongation rate. The elongation rates found for hadronic showers within different models $\left(D_{10}^{\text {had }} \approx 50 \ldots 60 \mathrm{~g} / \mathrm{cm}^{2}\right.$ at $\left.10^{19} \mathrm{eV}\right)$ can be qualitatively explained with the elongation rate theorem [156, 154]. An increase of the observed $\left\langle X_{\max }\right\rangle$ faster than or comparable to the elongation rate of em. showers is a model-independent signature for a transition to a lighter composition. However, over a limited range in energy, drastic changes in the characteristics of hadronic multiparticle production can also lead to an elongation rate comparable to that of em. showers [186].

Finally, it should be mentioned that the em. shower component exhibits a number of universality features that are independent of the primary hadron type and also rather insensitive to the primary energy. For example, near the shower core, the electron energy distribution is a universal function of shower age and the angular distribution of electrons depends almost exclusively on the electron energy and only slightly on shower age [189, 190, 191]. Furthermore, the longitudinal shower profile (particle flux) at a given lateral distance can be well parametrized by an universal function of the depth of shower maximum and shower size at maximum [192]. This reflects the universality of the lateral distribution of electromagnetic particles if the lateral distance is measured in Moliere units [194].

\section{Measurement of Charged Secondary Particles}

\section{Detection Techniques}

The classical set-up to measure air showers is an array of scintillation detectors, registering charged particles from the shower. In each detector the density of charged particles (mostly 
electrons, positrons, but also some converted photons) is measured. From this information the lateral distribution of the electromagnetic component is inferred. This yields information on the position of the shower core and the total number of particles in the shower. Measurements of the arrival times of the particles with a resolution of a few ns allow to reconstruct the orientation of the shower plane and thus, perpendicular to it, the arrival direction of the primary cosmic ray. Due to the large number of secondary particles it is usually sufficient to cover only a small fraction of the total area with detectors. Typical values range from $1.2 \%$ for the KASCADE array to $5 \times 10^{-6}$ for the Auger array.

Examples for arrays in the knee region are the EAS-TOP experiment [196], where 37 stations of scintillation detectors were distributed over an area of $\sim 10^{5} \mathrm{~m}^{2}$, located above the Gran Sasso underground laboratory at an altitude of $2005 \mathrm{~m}$, or the KASCADE experiment [197, where 252 detector stations are placed on a rectangular $13 \mathrm{~m}$ grid, covering an area of $200 \times 200 \mathrm{~m}^{2}$. Detectors that operate at higher energy are the scintillator array of the KASCADE-Grande experiment [198], or the AGASA experiment, which covered an area of about $100 \mathrm{~km}^{2}$ with 111 scintillation counter stations [37].

Another technique utilized to measure charged particles are water Cherenkov detectors, like the surface array of the Pierre Auger Observatory with its 1600 detectors distributed over an area of $3000 \mathrm{~km}^{2}$ [199]. They are relatively deep (typically $\sim 1 \mathrm{~m}$ ) compared to scintillators with typical thicknesses of the order of several $\mathrm{cm}$. Consequently they have a larger response to showers with large zenith angles, e.g. the FWHM of the declination distributions were $\sim 40^{\circ}$ and $\sim 75^{\circ}$ for the Volcano Ranch (scintillators) [200] and Haverah Park (water Cherenkov) 35] experiments respectively. This results in a much larger sky coverage of a water Cherenkov experiment compared to a scintillator array. They are also suitable for the measurement of horizontal showers that can be used to detect neutrinos at the highest energies.

Various techniques are applied for the detection of muons in air showers. Frequently, particle counters are covered with absorbers of lead, iron, or soil with a thickness of a few tens $X_{R}$ to suppress the electromagnetic component, as in the KASCADE [197] or AGASA [37] experiments. Muons can also be identified via their trajectories in tracking devices, like the HEGRA CRT detectors [201] or the muon tracking detector of KASCADE [202]. In underground laboratories, well shielded by rock, soil, water, or ice absorbers with a thickness corresponding to several $1000 \mathrm{~m}$ w.e. (meter water equivalent), muons with thresholds in the $\mathrm{TeV}$ regime are registered 203.

The energy of hadrons is usually determined with calorimeters. The principle idea is to absorb an incoming particle and to measure the dissipated energy. Examples are the hadron calorimeters of the EAS-TOP [205] or KASCADE experiments [206].

\section{Measured Parameters}

The direction of the shower axis and hence of the primary cosmic ray is obtained by measurements of the arrival time of the shower front in the detector plane. The shower direction is the normal to the reconstructed, slightly curved shower front. Typical detectors have a time resolution in the range from $0.5 \mathrm{~ns}$ up to a few ns. The angular resolution ( $68 \%$ value) of the KASCADE detector field decreases from $0.55^{\circ}$ for small showers with $N_{e} \approx 10^{4}$ to $0.1^{\circ}$ for showers with $N_{e} \approx 10^{6}$ [197]. The angular resolution of the Auger surface detectors decreases from values around $2.2^{\circ}$ for showers with energies below $4 \mathrm{EeV}$ to values from $0.5^{\circ}$ to $1^{\circ}$, depending on the zenith angle, for higher energies (>10 EeV) [207].

To obtain the position of the shower core and the number of particles, the particle densities are measured and an appropriate lateral distribution function is fitted to the data. The number of particles is calculated by integration of the measured lateral distribution. Historically, the choices of parameterizations of the electron and muon lateral distributions were influenced by a 
review by Greisen [138]. The Nishimura-Kamata-Greisen (NKG) function became the standard function to describe the particle density $\rho$ for a shower with the number of electrons $N_{e}$ and shower age $s$ as function of distance $r$ to the shower, see Eq. (7) [132, 138]. Traditionally, a fixed Molière unit $r_{1}=79 \mathrm{~m}$ is used and the parameter $s$ is limited to $0.5<s<1.5$. To parameterize the lateral distribution of muons, Greisen suggested the function [138]

$$
\rho_{\mu}\left(r, N_{\mu}\right)=C N_{\mu}\left(\frac{r}{r_{G}}\right)^{-\beta}\left(1+\frac{r}{r_{G}}\right)^{-2.5}
$$

to describe the density of muons $\rho_{\mu}$ as function of distance $r$ to the shower axis for a shower with a total muon number $N_{\mu}$. The Greisen radius is $r_{G}=320 \mathrm{~m}$.

The KASCADE group found that the lateral distributions of all three major shower components (electromagnetic, muonic, and hadronic) can be parameterized using the NKG function 208]. Fitting simultaneously the parameters $N_{e}, r_{1}$, and $s$, the measured lateral distributions of the electromagnetic component can be reproduced with an accuracy of about 1\%, yielding $r_{1}^{e} \approx 20-30 \mathrm{~m}$. The experimental muon distributions are described with an accuracy of $5 \%$ using $r_{1}^{\mu}=420 \mathrm{~m}$. Finally, for the lateral distribution of hadrons with energies above $50 \mathrm{GeV}$ a value $r_{1}^{h} \approx 10 \mathrm{~m}$ has been found.

The NKG function has been analytically developed to describe pure electromagnetic showers. For hadron-induced air showers it exhibits shortcomings describing the measured electron lateral distributions, most obvious at large core distances. Investigations of the KASCADE group showed that the measured electron lateral distributions for showers with energies up to $10^{17} \mathrm{eV}$ and core distances up to $200 \mathrm{~m}$ can be described better using a modified NKG function [209]

$$
\rho_{N K G}^{m o d}=N_{e} \cdot c(s) \cdot\left(\frac{r}{r_{0}}\right)^{s-\alpha}\left(1+\frac{r}{r_{0}}\right)^{s-\beta} \text { with } c(s)=\frac{\Gamma(\beta-s)}{2 \pi r_{0}^{2} \Gamma(s-\alpha+2) \Gamma(\alpha+\beta-2 s-2)} .
$$

Optimizing the parameters with Monte Carlo data, the values $\alpha=1.5$ and $\beta=3.6$ have been obtained, when $r_{0}=40 \mathrm{~m}$ is used for the scale parameter.

For showers in the EeV energy range, the AGASA group uses the parameterization

$$
\rho(r)=C\left(\frac{r}{r_{M}}\right)^{-1.2}\left(1+\frac{r}{r_{M}}\right)^{-(\eta-1.2)}\left(1.0+\left(\frac{r}{1000 \mathrm{~m}}\right)^{2}\right)^{-\delta}
$$

to describe the lateral distribution of charged particles up to distances of several $\mathrm{km}$ from the shower axis [210, inspired by a function suggested by Linsley [211]. The parameters are $\eta=3.8$, $\delta=0.6 \pm 0.1$ and a Molière unit $r_{M}=91.6 \mathrm{~m}$ for near vertical showers with $\sec \theta<1.2$.

Another parameterization for the electron lateral distribution has been suggested by the Haverah Park Collaboration [212]. It is also applied in the Auger Observatory [213]. The signal $S$ in a water Cherenkov detector is parameterized as function of distance $r$ to the shower core as $S(r)=k r^{-\left(\eta+r / r_{s}\right)}$ for $r<800 \mathrm{~m}$ and $S(r)=(1 / 800)^{\delta} k r^{-\left(\eta+r / r_{s}\right)+\delta}$ at larger distances. The shape parameter $\eta$ varies with zenith angle, while the parameter $\delta$ and the scale radius $r_{s}=4000 \mathrm{~m}$ are fixed.

The position of the shower core is determined by the KASCADE group with an uncertainty of $5 \mathrm{~m}$ for showers with an electron number $N_{e} \approx 10^{4}$ improving to less than $1 \mathrm{~m}$ for large showers $\left(N_{e} \approx 10^{6.5}\right)$ [197. The error in the reconstructed number of electrons decreases in the same electron number interval from $18 \%$ to less than $4 \%$. Similar values were obtained in the EAS-TOP experiment [196]. In the electron number range from $N_{e}=10^{4.8}$ to $10^{6.8}$, the error in the position of the shower core improves from 7.5 to $2 \mathrm{~m}$ and the uncertainty in the number of electrons decreases from $\Delta N_{e} / N_{e}=28 \%$ to about $10 \%$. 


\section{Energy Estimators}

One of the most important parameters to characterize a shower is the energy of the primary particle. Various methods are discussed in the literature to obtain this value.

For KASCADE, it has been found that, at sea level, the number of muons with energy above $230 \mathrm{MeV}$ in the range from $40 \mathrm{~m}$ to $200 \mathrm{~m}$ from the shower axis is a good measure for the primary energy independent of the mass of the primary particle [214]. With its relatively high detector density the lateral distribution of muons is measured very well in the radius range from 40 to $200 \mathrm{~m}$. Extrapolating beyond these limits would introduce uncertainties related to the (less well known) shape of the lateral distribution. Hence, the number of muons is reconstructed using a distance range only, in which detectors are present.

Another method is to use the correlation between the number of electrons and muons reconstructed. For example the CASA-MIA group uses the relation $E_{0}=0.8 \mathrm{GeV}\left(N_{e}+25 N_{\mu}\right)$ to estimate the primary energy [215]. Similarly, the KASCADE-Grande experiment obtained the relation

$$
\log _{10}\left(\frac{E_{0}}{\mathrm{GeV}}\right)=0.313 \log _{10} N_{e}+0.666 \log _{10} N_{\mu}+1.24 / \cos \theta+0.580
$$

to estimate the primary energy as function of the observed number of electrons $\left(E_{e}>3 \mathrm{MeV}\right)$ and muons $\left(E_{\mu}>300 \mathrm{MeV}\right)$ at sea level for showers with zenith angle $\theta$ [216].

A similar method has been applied by the AGASA group [217]. Here, the particle density as measured $600 \mathrm{~m}$ from the shower core is used to estimate the primary energy. At this distance the fluctuations in the lateral distribution are found to be relatively small [218, 219]. The measured value is corrected for attenuation due to the zenith angle and converted to a value $S_{0}(600)$ for vertically incident showers. The conversion to primary energy via the relation $E[\mathrm{eV}]=2.23 \times 10^{17} \cdot S_{0}(600)^{1.02}\left[\mathrm{~m}^{2}\right]$ yields at $10^{20} \mathrm{eV}$ a difference between proton and iron induced showers of about $10 \%$ and a similar difference is obtained using two different codes to describe hadronic interactions in the atmosphere, namely QGSJET and SIBYLL [220].

The methods described so far depend on simulations of the shower development in the atmosphere. To avoid this uncertainty another method is being applied in the Auger Observatory [221]. It makes use of the constant intensity method, which relies on the fact that primary cosmic rays arrive isotropically. The value $S(1000)$ of the measured signal in a water Cherenkov detector at a distance of $1000 \mathrm{~m}$ from the shower axis is used. The dependence of this parameter on the depth in the atmosphere (which varies as $\sec \theta$ of the shower zenith angle $\theta$ ) is obtained from the measured data. Using this dependence, the actually measured signal is converted to the value $S_{38}$, representing the signal for a shower with a zenith angle of $38^{\circ}$. Finally, the primary energy is estimated as $E[\mathrm{EeV}]=0.149 \cdot S_{38}^{1.08}$ using the fluorescence telescopes as optical calorimeters, which define the energy scale in a direct way (see Sec.2] [221].

\section{Composition Estimators}

To estimate the mass of the shower-inducing primary particle the following array observables are used: the electron-to-muon number ratio, the arrival time distribution of the particles, the curvature of the shower front, and the slope of the lateral distribution.

The method applied most frequently is the measurement of the electron-to-muon ratio at ground level. Plotting the number of electrons $N_{e}$ and muons $N_{\mu}$ in a plane as shown in Fig.5, we find an energy axis (in the direction of the main diagonal) and (almost perpendicular to it) a mass axis. Using a Heitler cascade model the ratio of electrons to muons at shower maximum can be estimated, yielding the relation 153 .

$$
\frac{N_{e}}{N_{\mu}}=\left(\frac{E_{0}}{A \cdot 1 \mathrm{PeV}}\right)^{0.15} \text { or } \log _{10}\left(\frac{N_{e}}{N_{\mu}}\right)=C-0.065 \ln A .
$$


This illustrates that $\log _{10} N_{e} / N_{\mu}$ is a function of $\ln A$. It can be estimated that an uncertainty in $\log _{10}\left(N_{e} / N_{\mu}\right)$ of about $16 \%$ results in an uncertainty of about one unit in $\ln A$.

Also the shape of the shower front or the arrival time distribution of the particles at ground level is utilized as an indirect estimate of the depth of the shower maximum. For heavy nuclei, muons are produced earlier in the shower development and reach the ground also earlier as compared to the electromagnetic component whose particles follow the shower core and branch off to large lateral distances only very late before reaching the detector array. It is understood that the narrower pulse profiles correspond to higher production altitudes, which in turn lead to similar arrival times in the detectors (see e.g. [222, 223]).

\section{Measurement of Cherenkov Light}

Many particles in the shower disc travel with relativistic velocities through the atmosphere. Approximately one third of the charged particles emit Cherenkov light in the forward direction 224], the Cherenkov angle in air at sea level amounts to $1.3^{\circ}$ only. Electrons (and positrons) are the most abundant charged species in air showers. Due to their relatively low Cherenkov threshold (21 MeV at sea level), they contribute mostly to the Cherenkov light in air showers.

At present, for the detection of Cherenkov light, two techniques are applied: integrating detectors, in principle consisting of arrays of photomultipliers inside light collecting cones, looking upwards in the sky, and imaging detectors or telescopes, composed of large area collection mirrors and a camera with segmented read-out. Optical detectors such as Cherenkov detectors and fluorescence detectors (described in the next section) can only be operated during clear moon-less nights to obtain reliable data. This restricts their duty cycle to about $10 \%$.

\section{Light Integrating Detectors}

The basic idea of integrating detectors is to measure the lateral distribution of the Cherenkov light with an array of photomultipliers distributed over a large area on ground level. To enlarge the collection area, the PMTs are installed inside light collecting cones (Winston cones). Such observations yield the lateral density distribution of Cherenkov photons. It can be parameterized by the empirical relation

$$
C(r)= \begin{cases}C_{120} \cdot \exp (s[120-r / \mathrm{m}]) ; & 30 \mathrm{~m}<r \leq 120 \mathrm{~m} \\ C_{120} \cdot(r / 120 \mathrm{~m})^{-\beta} ; & 120 \mathrm{~m}<r \leq 350 \mathrm{~m}\end{cases}
$$

a combination of an exponential and a power law function [225]. $C_{120}$ is the Cherenkov light intensity at $120 \mathrm{~m}$ distance from the shower core, $s$ the exponential inner slope, and $\beta$ the outer slope. The energy of the primary particle is strongly correlated with the photon density at $120 \mathrm{~m}, C_{120}$ grows approximately as $E^{1.07}$. The average depth of the shower maximum $X_{\max }$ is approximately linearly related to the exponential slope $s$. Hence, from the Cherenkov measurements both, energy and mass of the primary particle can be derived. The latter through the dependence of the average depth of the shower maximum on the primary particle mass.

This technique was pioneered by the AIROBICC experiment on La Palma island [226]. Another example is the BLANCA instrument, which was located at the Dugway Proving Ground in Utah, USA and operated in coincidence with the CASA experiment at the same site [225]. BLANCA consisted of 144 angle-integrating detectors which recorded the lateral distribution of air shower Cherenkov light. A Cherenkov detector array is also installed in the Tunka valley close to lake Baikal in Siberia [227, 229], consisting of 25 detectors that cover an area of $0.1 \mathrm{~km}^{2}$. It is planned to extend this installation with 133 optical detectors covering an area of about $1 \mathrm{~km}^{2}$ [230]. 

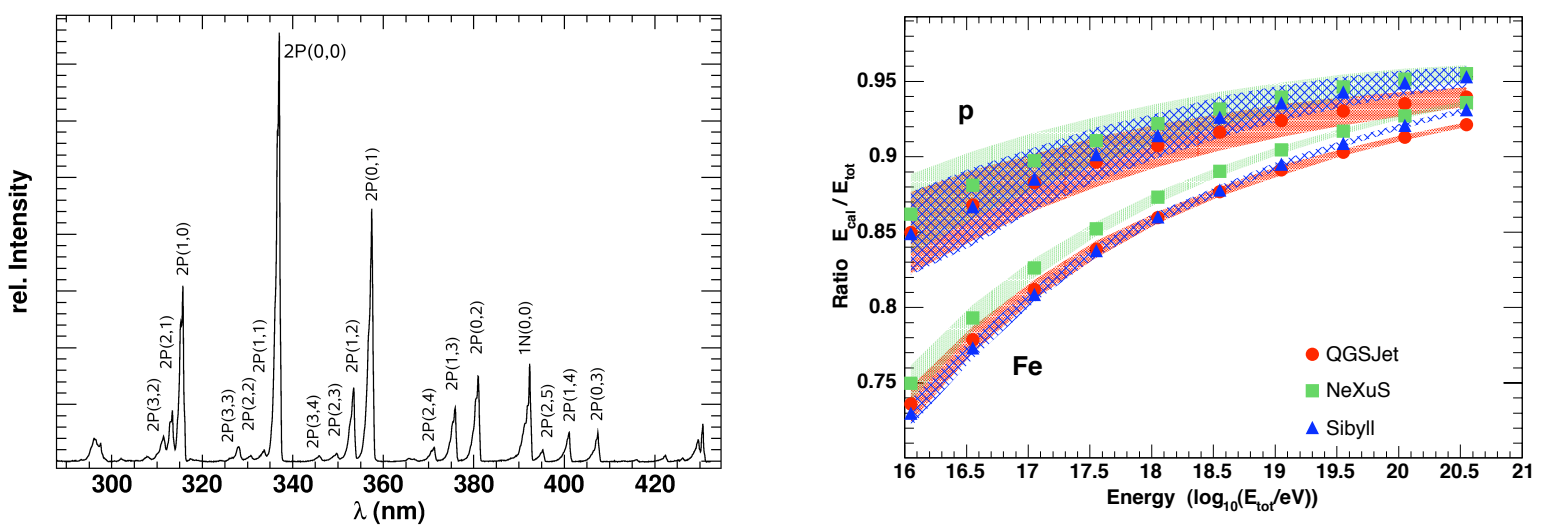

Figure 6: Left panel: Fluorescence light spectrum of air as measured by the AIRFLY experiment [236, 237] with an electron beam of $3 \mathrm{MeV}$. The measurement was done at $20^{\circ} \mathrm{C}$ and a pressure of $800 \mathrm{hPa}$. Right panel: Ratio of calorimetric to total shower energy [184]. Shown are predictions calculated with CONEX [183] and different highenergy interaction models. The symbols present the mean values and $1 \sigma$ fluctuations are indicated by the shaded bands.

\section{Imaging Cherenkov Detectors}

Cosmic-ray events within the field of view of an imaging atmospheric Cherenkov telescope produce a focal plane image which corresponds to the direction and intensity of Cherenkov light coming from the air shower. When the direction of the air shower core and the distance of the shower axis from the telescopes are known, simple geometry can be used to reconstruct the light received from each altitude of the shower. The amount of Cherenkov light produced is strongly correlated with the number of electrons in the shower and is used to estimate the shower size as a function of depth in the atmosphere from which the location of the shower maximum can be determined. This procedure is essentially geometrical and has the advantage of being almost independent of numerical simulations expect for the calculation of the angular distribution of Cherenkov light around the shower axis.

Large Cherenkov telescopes are used to reconstruct air showers initiated by primary gamma rays in $\mathrm{TeV} \gamma$-ray astronomy. The telescopes and analysis procedures are designed to effectively suppress the much more abundant (up to a factor of 1000) hadron induced showers. The technique was established by the pioneering work of the WHIPPLE telescope [231]. Among the presently largest installations are the H.E.S.S. [232], MAGIC [233], and VERITAS [234] telescopes.

An example for a Cherenkov telescope, optimized for the reconstruction of hadron induced showers was the Dual Imaging Cherenkov Experiment (DICE) 235. It was located inside the CASA-MIA array in Dugway, Utah (USA) and comprised two telescopes, each equipped with a $2 \mathrm{~m}$ diameter spherical mirror viewed by an array of 256 PMTs.

\section{Measurement of Fluorescence Light}

At very high energy $\left(E \gtrsim 10^{17} \mathrm{eV}\right)$, the fluorescence light technique can be used to measure directly the longitudinal profile of air showers. This technique is based on the detection of fluorescence light emitted by nitrogen molecules that are excited by charged particles traversing the atmosphere.

There are two transitions of electronic states of the nitrogen molecule, called $2 \mathrm{P}$ and $1 \mathrm{~N}$ for historical reasons, that lead - in combination with the change of the vibrational and rotational 
states of the molecule - to several fluorescence emission bands. A recent spectrometer measurement [236, 237] of these bands is shown in Fig. 6. The bands are labeled with the electronic transition type $(2 \mathrm{P}$ or $1 \mathrm{~N})$ and the change of the vibration quantum number $\left(\nu^{\prime} \rightarrow \nu^{\prime \prime}\right)$, for example, $2 \mathrm{P}\left(\nu^{\prime}, \nu^{\prime \prime}\right)$. Most of the fluorescence light emission is found in the wave length range between 300 and $400 \mathrm{~nm}$ (near UV). The lifetime of the excited states of nitrogen is of the order of $10 \mathrm{~ns}$ and the fluorescence light is emitted isotropically.

The fluorescence yield, the number of photons produced per deposited energy, depends on the gas mixture in the atmosphere and atmospheric conditions. Collisions between molecules give rise to competing de-excitation processes (collisional quenching, see, for example, discussion in [238]). The importance of quenching increases with pressure and almost cancels the density dependence of the energy deposit per unit length of particle trajectory. This results in a weakly height dependent rate of about 4-5 fluorescence photons produced per meter and charged shower particle at relevant altitudes. During the last years several experiments have been set up for measuring the fluorescence yield under different atmospheric conditions [239, 240, 241, 242, 236, 243, 244, 245]. Recent progress is reviewed in [246]. Still, the fluorescence yield is currently known only to a precision of about $15 \%$ [240] as end-to-end calibrated data are not yet available.

Recent measurements confirm the expectation that the fluorescence yield is independent of the energy of the exciting particle. Only at very low energy, deviations are expected [247. Therefore, if the atmospheric dependence of the fluorescence yield is taken into account, the fluorescence technique allows a calorimetric measurement of the energy deposited in the atmosphere. Simulations show that about $90 \%$ of the total shower energy is converted to ionization energy and, hence, is accessible for detection [248, 249, 184]. The average ratio between the energy deposited in the atmosphere and the primary particle energy is shown in Fig. 6 (right). It depends on the primary particle type and energy and, to some extent, also on the model used in the simulations. However, as most of the shower energy is transferred to em. particles, the model dependence corresponds to an uncertainty of only a few percent of the total energy. In case of a gamma-ray as a primary, about $99 \%$ of the energy is deposited in the atmosphere.

A complete reconstruction of a shower profile with the fluorescence technique requires the determination of the geometry of the shower axis, the determination of the Cherenkov light fraction, and the correction for the wavelength dependent atmospheric absorption of light.

In shower observations with one fluorescence telescope (monocular observation), a showerdetector plane is given by the image of the light track. The orientation of the shower within this plane follows from the time sequence of the PMT signals [38, 250]. The angular uncertainty of the orientation of the shower-detector plane depends on the resolution of the fluorescence camera and the length of the measured track. Typically a resolution of the order of $1^{\circ}$ is obtained. In general, the reconstruction resolution of the angle within the shower-detector plane is much worse and varies between $4.5^{\circ}$ and $15^{\circ}$ (for example, see [251]). The reconstruction accuracy can be improved considerably by measuring showers simultaneously with two telescopes (stereo observation). Showers observed in stereo mode can be reconstructed with an angular resolution of about $0.6^{\circ}$ [251. A similar reconstruction quality is achieved in hybrid experiments that use surface detectors to determine the arrival time of the shower front at ground [252, 207.

In fluorescence measurements, the Cherenkov light signal of air showers can be considered as a highly asymmetric background contribution but can also be exploited as an independent signal [253]. Knowing the longitudinal shower profile, the Cherenkov light contribution to the detected signal can be estimated using parametrized electron energy distributions and models for the angular distribution of the emitted Cherenkov light [38, 190, 191, 254].

In general, fluorescence detectors require continuous monitoring of atmospheric conditions, in particular the measurement of the wavelength dependent Mie scattering length and detection of clouds [255, 199, 256]. The temporal variations of the density profile of the atmosphere can lead to additional systematic uncertainties of shower reconstruction, in particular of the depth 
of shower maximum [257].

The first fully functional air shower fluorescence detector was the Fly's Eye experiment in Utah that began taking data in 1982 and was operated for 10 years [38. The Fly's Eye detector was a setup of two stations, Fly's Eye I (67 spherical mirrors of $1.5 \mathrm{~m}$ diameter, viewed by 880 PMTs in total) and Fly's Eye II ( 8 mirrors viewed by 120 PMTs). The Fly's Eye I detector had a total field of view (FoV) of $360^{\circ}$ in azimuth and $90^{\circ}$ in zenith. With the smaller FoV of about $90^{\circ}$ in azimuth and an elevation range from $2^{\circ}$ to $38^{\circ}$ degrees, the Fly's Eye II station was designed to measure showers in coincidence with Fly's Eye I. In October 1991 the shower of the highest energy measured so far, $E=(3.2 \pm 0.9) \times 10^{20} \mathrm{eV}$, was detected with Fly's Eye I 258.

The successor to the Fly's Eye experiment, the High Resolution Fly's Eye (HiRes), took data from 1997 (HiRes I) and 1999 (HiRes II) to 2006. The largest data set of HiRes is that of monocular observations with HiRes I, a telescope consisting of a ring of mirrors with a FoV from $3^{\circ}$ to $16^{\circ}$ in elevation and full azimuth. HiRes II is built up of two rings of mirrors covering elevation angles up to $30^{\circ}$. The optical resolution of the HiRes detectors is with $1^{\circ} \times 1^{\circ}$ per PMT much higher than that of Fly's Eye.

The Pierre Auger Observatory combines the observation of fluorescence light using imaging telescopes with the measurement of particles reaching ground level in a "hybrid approach" [199]. The southern Auger Observatory (near Malargue, Argentina) is the world's largest air shower detector and comprises 1600 polyethylene tanks set up in an area covering $3000 \mathrm{~km}^{2}$. Each water Cherenkov detector has $3.6 \mathrm{~m}$ diameter and is $1.55 \mathrm{~m}$ high, containing $12 \mathrm{~m}^{3}$ of highpurity water. A radio system is used to provide communication between each station and a central data acquisition system. Four telescope systems overlook the surface array. A single telescope system comprises six telescopes, overlooking separate volumes of air. Each telescope has a camera with 440 PMT pixels, whose field of view is approximately $1.5^{\circ}$. One camera overlooks a total field of view of $30^{\circ}$ azimuth $\times 28.6^{\circ}$ elevation.

In the northern hemisphere, the Telescope Array (TA) is located in Millard County, Utah, USA [259]. It covers an area of $860 \mathrm{~km}^{2}$ and comprises 576 scintillator stations and three fluorescence detector sites on a triangle with about $35 \mathrm{~km}$ separation, each equipped with twelve fluorescence telescopes.

\section{Measurement of Radio Emission from Air Showers}

An independent measurement technique to observe air showers is provided by means of detection of radio-frequency electromagnetic waves emitted from showers. Coherent radio emission generated by extensive air showers was theoretically predicted by Askaryan in 1961 [261] and experimentally discovered by Jelly et al. in 1965 at a frequency of $44 \mathrm{MHz}$ [262]. Over a period of time this phenomenon has been considered as an interesting alternative to traditional methods of detection of high-energy cosmic rays with energy greater than $10^{17} \mathrm{eV}$. In the $1960 \mathrm{~s}$ and $1970 \mathrm{~s}$ the experimental and theoretical efforts in this direction had only limited success [263. Modern experiments, such as CODALEMA [264] and LOPES [265], aim at studies of radio emission from air showers using modern, improved instruments. First break-throughs have been achieved [266, 267]. At present, also activities are under way to install prototype antenna systems at the site of the Pierre Auger Observatory in Argentina to investigate the possibility for radio detection of air showers at the highest energies [268]. For a review of recent developments, see 269.

In addition to experimental difficulties there remain questions concerning the quantitative radio emission theory. Several mechanisms of radio emission generation in air have been identified after the pioneering work of Askaryan where the coherent Cherenkov radiation of the chargeexcess was put forward [261]. This radiation is very strong for showers developing in dense media 


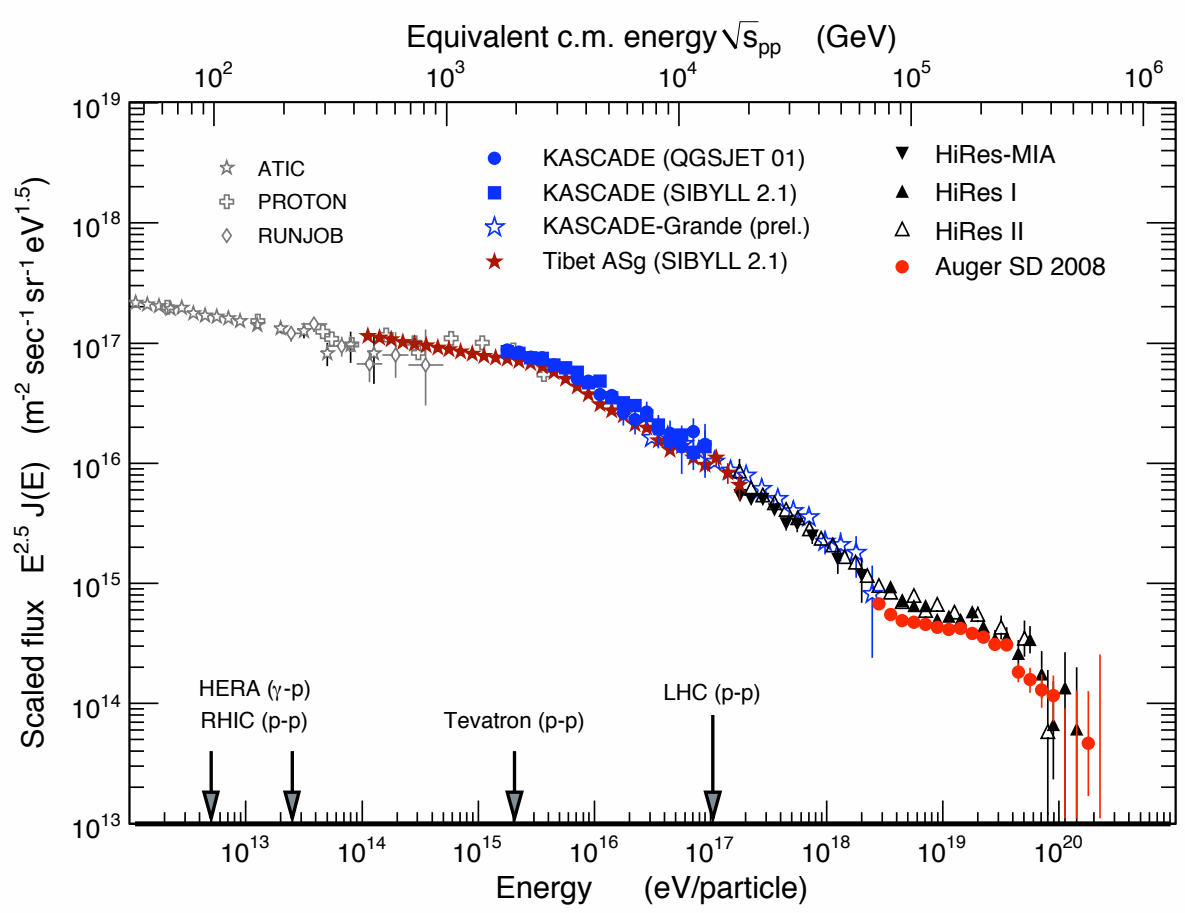

Figure 7: All-particle cosmic-ray energy spectrum as obtained by direct measurements above the atmosphere by the ATIC [280, 281], PROTON [282], and RUNJOB [284] as well as results from air shower experiments. Shown are Tibet AS $\gamma$ results obtained with SIBYLL 2.1 [285], KASCADE data (interpreted with two hadronic interaction models) [286], preliminary KASCADE-Grande results [287], and Akeno data [288, 42]. The measurements at high energy are represented by HiRes-MIA [289, 290], HiRes I and II [291], and Auger [221].

[270. In the case of air showers there is also an alternative radiation due to the acceleration of charged shower particles in the Earth's magnetic field. It is called geosynchrotron mechanism and has been recently investigated in detail [273]. The interrelation between these two essential mechanisms is not clear at present. Hence, also combined efforts are in progress, performing accurate radio emission calculations within the framework of a unified approach [276].

\section{$3 \quad$ Energy Spectra}

The all-particle energy spectrum extending from $10^{12} \mathrm{eV}$ up to the highest energies is shown in Fig.77. The flux as obtained from direct measurements above the atmosphere (represented in the figure through results from ATIC, PROTON, and RUNJOB) extends smoothly to high energies in the air shower detection regime. The all-particle spectrum can be approximated by a broken power law $\propto E^{\gamma}$ with a spectral index $\gamma=-2.7$ below $E_{k} \approx 4 \times 10^{15} \mathrm{eV}$. At this energy, the knee, the spectral index changes to $\gamma \approx-3.1$.

In the following we consider in more detail two energy regions: galactic cosmic rays up to energies of about $10^{17}$ to $10^{18} \mathrm{eV}$ and the extragalactic component at higher energies. ${ }^{4}$

\footnotetext{
${ }^{4}$ The exact energy of the transition from galactic to extragalactic cosmic rays is presently not known, however, it is generally assumed to be in the energy range indicated, see also Sec.6.
} 


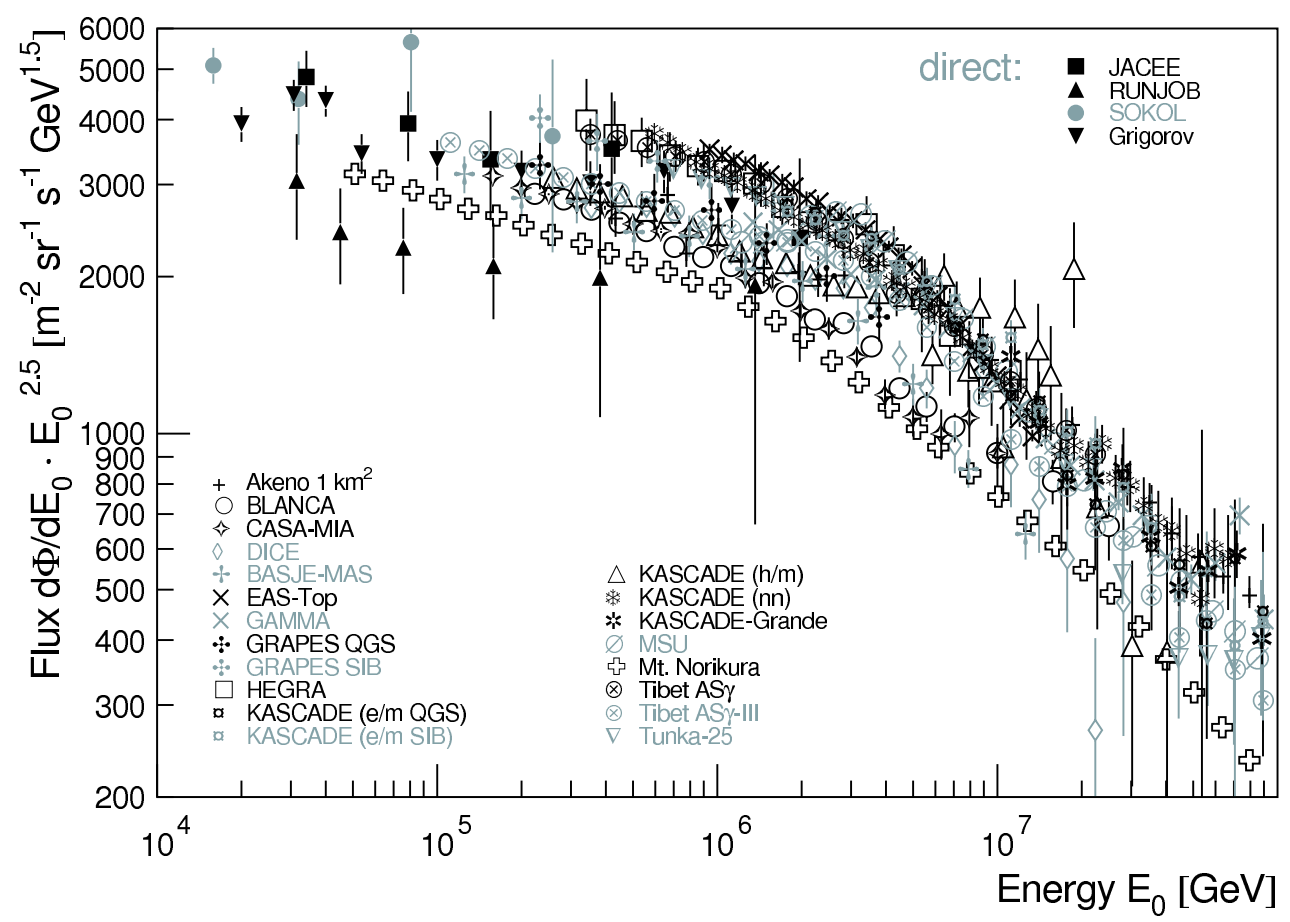

Figure 8: All-particle energy spectra in the knee region. Results from direct measurements by Grigorov et al. [282], JACEE [292], RUNJOB [284], and SOKOL [293] as well as from the air shower experiments Akeno $1 \mathrm{~km}^{2}$ [288], BASJE-MAS [294], BLANCA [225], CASA-MIA [215], DICE [235], EAS-TOP [295], HEGRA [226], KASCADE electrons and muons interpreted with two hadronic interaction models [286], hadrons [296, and a neural network analysis combining different shower components [297], MSU [298], Mt. Norikura [299], Tibet AS $\gamma$ [300] and AS $\gamma$-III [285], as well as Tunka-25 229].

\section{Galactic Cosmic Rays}

Many groups published results on the all-particle energy spectrum from indirect measurements in the knee region $\left(\approx 10^{15} \mathrm{eV}\right)$. The spectra obtained are compiled in Fig. 8. The ordinate has been multiplied by $E_{0}^{2.5}$. The individual measurements agree within a factor of two in the flux values and a similar shape can be recognized for all experiments with a knee at energies of about $4 \mathrm{PeV}$. Also shown are results for the all-particle flux as obtained by direct observations above the atmosphere approaching energies up to $1 \mathrm{PeV}$. In the region of overlap, the results from direct and indirect measurements are in reasonable agreement. Typical values for the systematic uncertainties of the absolute energy scale of air shower experiments are about 15 to $25 \%$. Renormalizing the energy scales of the individual experiments to match the all-particle spectrum obtained by direct measurements in the energy region up to almost a $\mathrm{PeV}$ requires correction factors of the order of $10 \%$ [3]. A remarkable result, indicating that behind an absorber of 11 hadronic interaction lengths or 30 radiation lengths the energy of the primary particle is determined with an absolute error of the order of $10 \%$. One should keep in mind that the experiments investigate different air shower components, are situated at different atmospheric depths, and use different interaction models to interpret the observed data. Nevertheless, the systematic differences are relatively small and the all-particle spectrum seems to be well known.

Up to about a $10^{15} \mathrm{eV}$ direct measurements have been performed with instruments above the atmosphere. As examples, results for primary protons, helium, and iron nuclei are compiled in Fig.9. Recently, also indirect measurements of elemental groups became possible, as discussed 


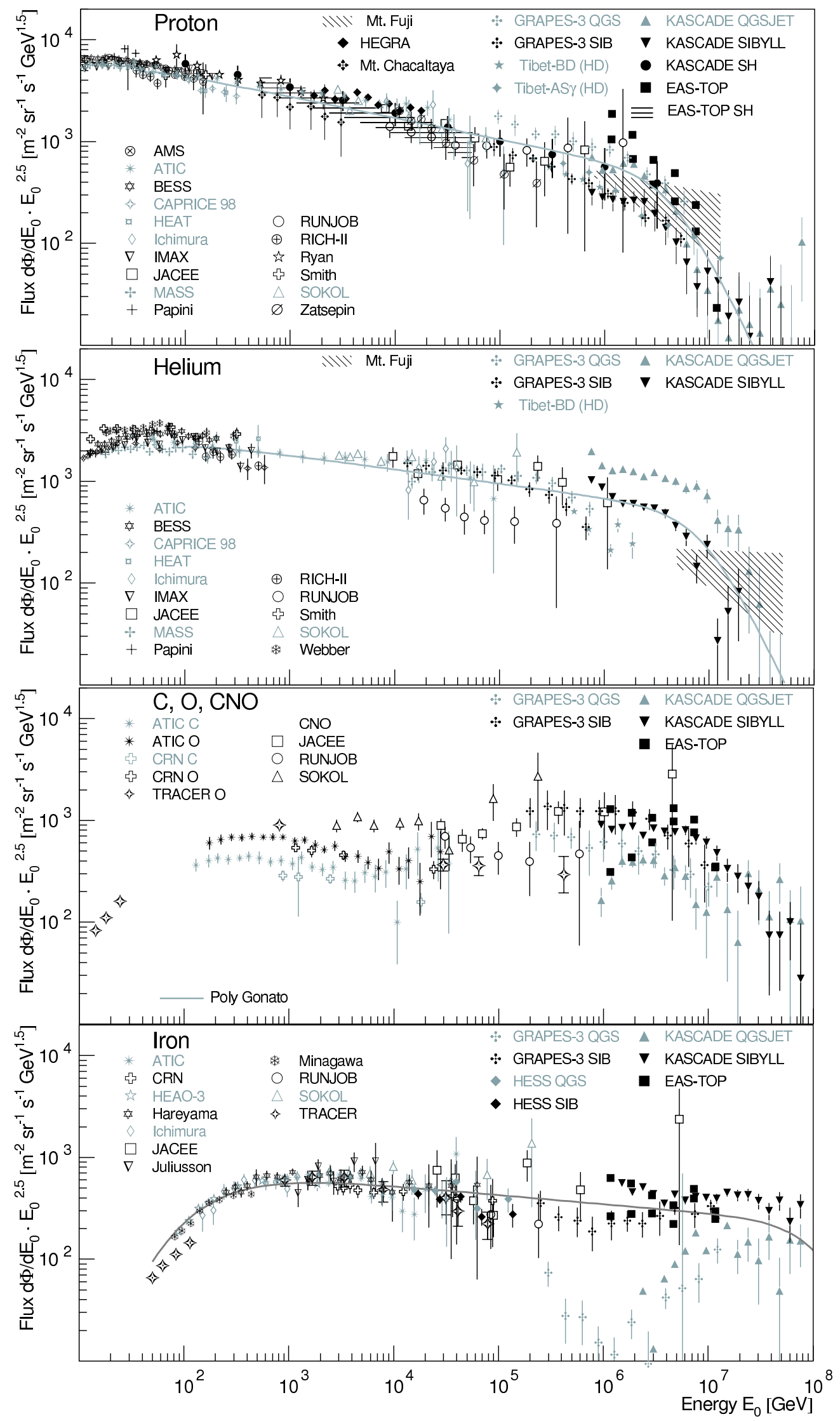

Figure 9: Energy spectra for elemental groups, caption see next page. 
Figure 9, Cosmic-ray energy spectra for four groups of elements, from top to bottom: protons, helium, CNO group, and iron group.

Protons: Results from direct measurements above the atmosphere by AMS [301, ATIC [302, BESS [303], CAPRICE [304, HEAT [305], [306], IMAX [307], JACEE [308], MASS [309], [310], RUNJOB [284], RICH-II [311], [312], [313], SOKOL [293], [314, and fluxes obtained from indirect measurements by KASCADE electrons and muons for two hadronic interaction models [286] and single hadrons [315], EAS-TOP (electrons and muons) [316] and single hadrons [317, GRAPES-3 interpreted with two hadronic interaction models [318, HEGRA [319], Mt. Chacaltaya [320, Mts. Fuji and Kanbala [321], Tibet burst detector (HD) 322] and $\mathrm{AS} \gamma$ (HD) 323.

Helium: Results from direct measurements above the atmosphere by ATIC [302, BESS [303, CAPRICE [304], HEAT [305], 306], IMAX [307], JACEE [308], MASS [309], 310], RICH-II [311], RUNJOB [284, [313], SOKOL [293], 324], and fluxes obtained from indirect measurements by KASCADE electrons and muons for two hadronic interaction models [286], GRAPES-3 interpreted with two hadronic interaction models [318, Mts. Fuji and Kanbala [321, and Tibet burst detector (HD) 322. CNO group: Results from direct measurements above the atmosphere by ATIC $(\mathrm{C}+\mathrm{O})$ [325], CRN $(\mathrm{C}+\mathrm{O})$ [326], TRACER (O) [327], JACEE (CNO) [328], RUNJOB (CNO) 284, SOKOL (CNO) 293, and fluxes obtained from indirect measurements by KASCADE electrons and muons [286], GRAPES-3 [318], the latter two give results for two hadronic interaction models, and EAS-TOP [316].

Iron: Results from direct measurements above the atmosphere by ATIC [325], CRN [326], HEAO-3 329], 330, 331, TRACER [327] (single element resolution) and [332, 306], JACEE [292, RUNJOB 284, SOKOL 293] (iron group), as well as fluxes from indirect measurements (iron group) by EAS-TOP [316], KASCADE electrons and muons [286], GRAPES-3 [318], and H.E.S.S. direct Cherenkov light 333]. The latter three experiments give results according to interpretations of the measured airshower data with two hadronic interaction models, namely QGSJET and SIBYLL. The gray solid lines indicate spectra according to the poly-gonato model [3].

below and the results of the KASCADE and EAS-TOP experiments are shown in the figures as well. Also results from other air shower experiments are shown. HEGRA used an imaging Cherenkov telescope system to derive the primary proton flux [319]. Spectra for protons and helium nuclei are obtained from emulsion chambers exposed at Mts. Fuji and Kanbala [321]. The Tibet group performs measurements with a burst detector as well as with emulsion chambers and an air shower array [322, 334]. GRAPES-3 uses the correlation between the registered number of electrons and muons to derive energy spectra for mass groups [318. The H.E.S.S. Cherenkov telescope system derived for the first time an energy spectrum measuring direct Cherenkov light 333. This light is emitted by the primary nuclei in the atmosphere before its first interaction, i.e. before the air shower begins [335]. Results for iron nuclei are shown.

Over the wide energy range depicted, the flux as obtained by direct measurements is smoothly continued to higher energies with the results of air shower measurements. Despite of the experimental uncertainties and systematic differences between different experiments and different interpretations of air shower data using various air shower models, a clear picture of the spectra for elemental groups is evolving. It is evident that the knee in the all-particle spectrum is caused by a depression of the flux of light elements. The measurements follow power laws with a cut-off at high energies. The spectra according to the poly-gonato model are indicated in the figures as lines. It can be recognized that the measured values are compatible with cut-offs at energies proportional to the nuclear charge $\hat{E}_{Z}=Z \cdot 4.5 \mathrm{PeV}$ [3, 336]. 


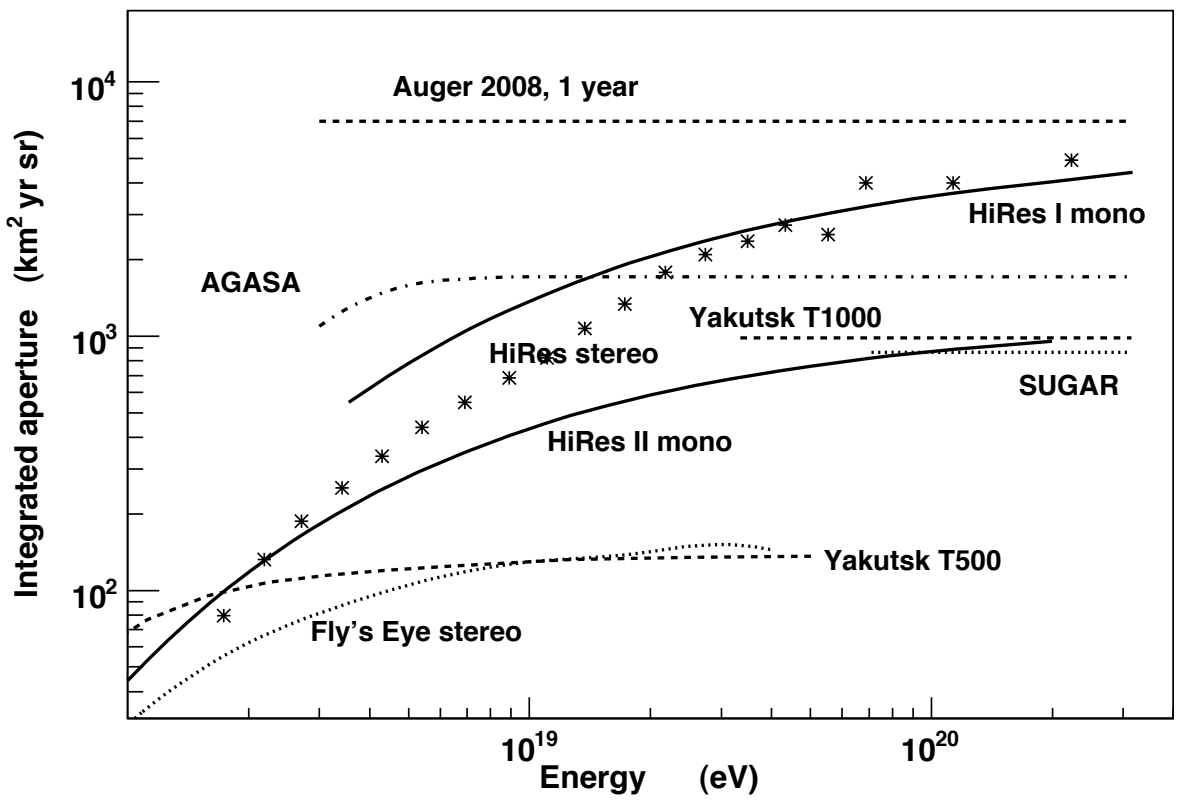

Figure 10: Integrated aperture of different high-energy detectors corresponding to the data shown in Fig. 11. The AGASA aperture refers to all air shower data with $\theta<45^{\circ}$ up to May 2003. The two HiRes detectors have different data taking periods: HiRes I from June 1997 to June 2005 and HiRes II from December 1999 to August 2004 [291]. The HiRes stereo exposure is that used for the recent anisotropy study [337]. The Auger exposure of $7000 \mathrm{~km}^{2}$ sr yr refers to data taking during the construction from Jan 2004 until August 2007, excluding events close to the array boundary [221]. The integrated aperture of the Yakutsk array includes data taken from September 1974 to June 2001 for T1000, and September 1979 till June 2001 for T500 [338]. The exposure shown for SUGAR is based on the re-analysis of the 5 highest energy events reported in [339] and corresponds to 11 years of operation. The Fly's Eye exposure in stereo mode is taken from [340. The integrated aperture of the data set used in 341] for calculating the Haverah Park flux is $7.39 \times 10^{12} \mathrm{~m}^{2} \mathrm{~s} \mathrm{sr}$.

\section{Extragalactic Cosmic Rays}

There are several detector installations that were designed to measure cosmic rays at the highest energies. At the high-energy end, the total aperture and observation time determine the statistics of expected events. At low energy, the acceptance range is given by the employed detection technique and typically the distance between the detectors.

A compilation of the integrated aperture (i.e. total exposure) reported by experiments with data above $10^{19} \mathrm{eV}$ is shown in Fig. 10. At ultra high-energy, about two times more events are expected in the Auger data set than HiRes has collected in monocular or stereo mode. The Yakutsk experiment is expected to have slightly more than a fourth of the statistics of HiRes I and about one half of that of AGASA.

The measurements of the cosmic-ray fluxes obtained with these exposures are shown in Fig. 11 (error bars indicate statistical uncertainties only). In case several analyses of the same data set are available, only the most recent results are included in the plot. The shaded area, depicting the results of the analysis of the Haverah Park data [341], accounts for some systematic uncertainties by assuming extreme elemental compositions, either fully iron or proton dominated. The highest energy point (Fly's Eye monocular observation) corresponds to the highest energy 


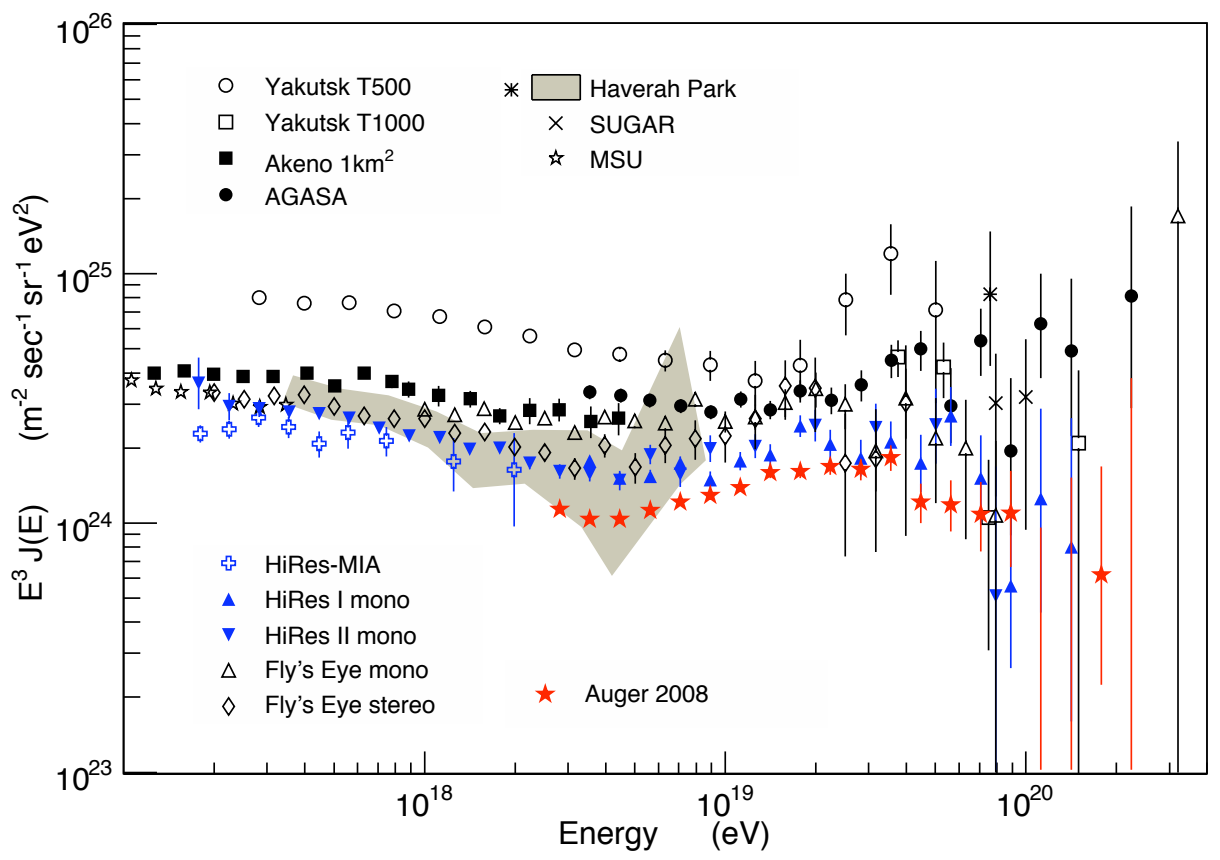

Figure 11: Comparison of flux measurements scaled by $E^{3}$. Only statistical errors are shown. Shown are the data of AGASA [217, 342], Akeno [42, 288], Auger [221], Fly's Eye [340, 40], Haverah Park [341, HiRes-MIA [289, 290], HiRes Fly's Eye [291], MSU [343], SUGAR [339], and Yakutsk [344]. Yakutsk T500 (trigger 500) refers to the smaller sub-array of the experiment with 500m detector spacing and T1000 (trigger 1000 ) to the array with $1000 \mathrm{~m}$ detector distance. The data of the MSU array are included to show the connection of the high-energy measurements to lower energy data covering the knee of the cosmic-ray spectrum.

event 258]. For sake of clarity upper limits are not shown.

It is common to present the data multiplied by $E^{3}$ to enhance deviations from a $d N / d E \sim$ $E^{-3}$ power law. In this representation, characteristic features such as the second knee at about $10^{17.5} \mathrm{eV}$ and the ankle at $10^{18.5} \mathrm{eV}$ are better visible. However, it should be emphasized that scaling the flux with energy to some power (e.g. $E^{3} J(E)$ or $E^{2.5} J(E)$ ) is misleading and does not reflect correctly the uncertainties of the measurements. In this presentation, the statistical uncertainties cannot be separated from the systematic energy calibration uncertainties. The importance of the systematic uncertainty of the experimental energy scale is demonstrated in Fig. 12 in which fluxes with the nominal energy scale of the experiments are compared with that after a model-motivated energy shift has been applied [347, 345]. After shifting the energy scales of the experiments, a very good overall agreement of the different measurements is obtained. In particular, the ankle in the cosmic-ray spectrum is clearly seen. The good agreement is a non-trivial observation as the position of the ankle and the overall flux change in a correlated way in the $E^{3}$-representation.

The data sets of the HiRes and Auger measurements provide evidence for a flux suppression at ultra high-energy. The statistical significance of the flux suppression is difficult to specify unambiguously. If one compares to a power-law flux a significance of more than $5 \sigma$ is found in each of the data sets [221, 291]. Both spectra can be well described by models with uniformly distributed sources and a GZK suppression [348, 349]. A similarly good agreement between GZK model predictions and the Yakutsk data was shown by several authors (for example, [347, 350]).

Only the AGASA data seem to disfavor a flux suppression. Assuming uniformly distributed sources of UHE protons and treating the normalization of the expected energy spectrum as free 

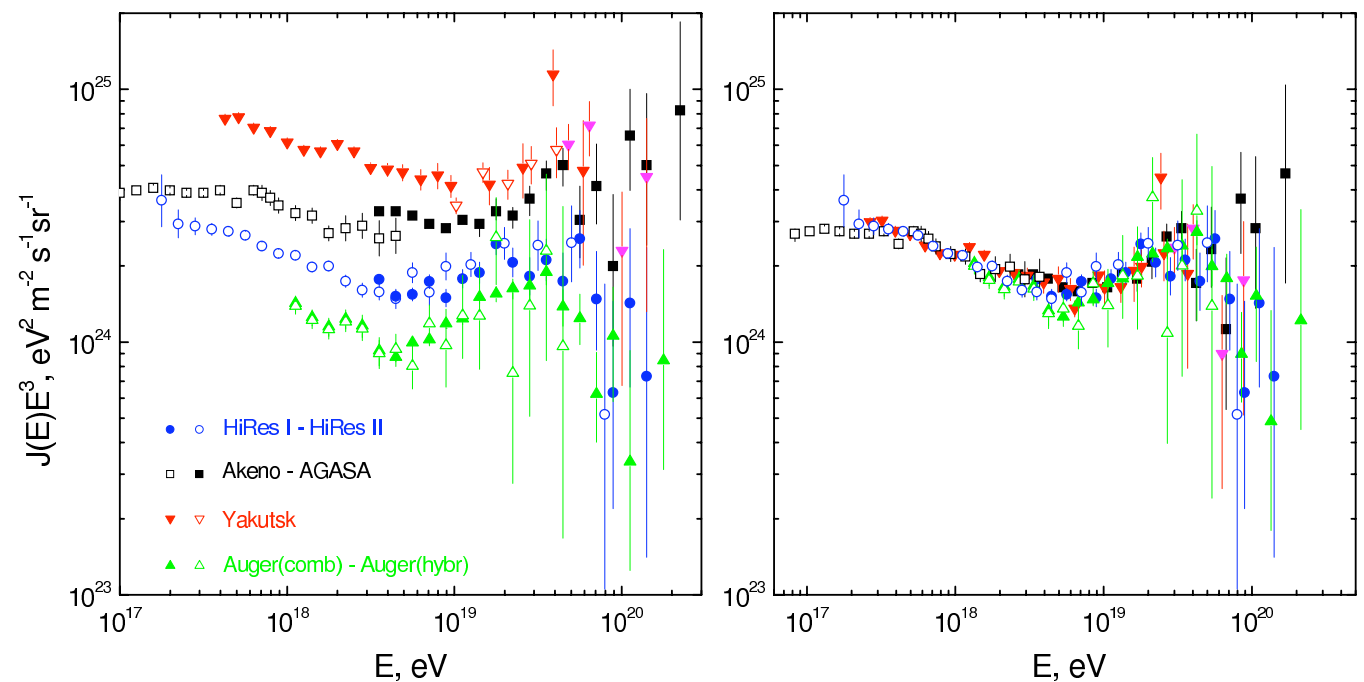

Figure 12: Flux of UHECRs as measured with the four detectors that have the largest exposures, namely Yakutsk [344] AGASA [217, 342], Auger [221], and HiRes [291]. Left panel: Cosmic-ray spectra as derived by the Collaborations using the calibration of the detectors. Right panel: Cosmic-ray spectra after re-scaling of the energy scale of the experiments to obtain a common position of the dip, from [345, 346]. The nominal energy scales of the experiments have been multiplied by 1.2, 1.0, 0.75, 0.625 for Auger, HiRes, AGASA, and Yakutsk, respectively.

parameter AGASA expects to observe 1.8 events above $10^{20} \mathrm{eV}$ with 11 actually detected. This corresponds to a $4.5 \sigma$ deviation from the GZK cutoff spectrum [342]. Other assumptions on the shape of the GZK proton spectrum lead to the prediction of 2.4 expected events [351, 350], corresponding to a deviation of $3.9 \sigma$ from the GZK cutoff hypothesis. Due to uncertainties in the absolute energy scale and partially also low statistics, the differences between the spectra of the different experiments are of limited statistical significance only [352, 353].

It should be kept in mind that a sudden and drastic change of composition from hadrons to photons could give observational results similar to a suppression of the flux 354 observed with fluorescence detectors, but not with surface arrays. So far there are no indications for very deeply penetrating showers that would be expected in such a case.

Given the importance of the absolute energy assignment to a reconstructed shower we will summarize the current systematic uncertainties below.

The energy reconstruction of showers detected with the AGASA array is based on the scintillator signal $S(600)$ at $600 \mathrm{~m}$ from the shower core, where shower-to-shower fluctuations are the smallest and the relation between the signal and the primary energy is almost composition independent [219, 355]. The systematic error of energy assignment is analyzed in [217] in detail, see also discussion [356. AGASA finds a total systematic uncertainty of the energy assignment of about $18 \%$. The main sources of uncertainty are related to shower phenomenology and the simulation of the relation of $S(600)$ to the primary particle energy. In particular, the observed discrepancy between the surface detector signal at $1000 \mathrm{~m}$ from the shower core and the fluorescence-based calorimetric energy measurement reported by Auger [357] indicates that currently available shower simulations do not allow to obtain an absolute energy scale with an systematic uncertainty smaller than 20\%. Therefore it is not surprising that discrepancies between the experimentally observed attenuation length for $S(600)$ and that expected from simulations hamper a re-analysis of the AGASA data 359.

The shower energy determination applied in HiRes is based on the track length integral $E_{\text {cal }}=\alpha_{\text {eff }} \int N(X) d X$, where $N(X)$ is a fit to the shower profile using the Gaisser-Hillas function 
(6) and $\alpha_{\text {eff }}$ denotes the mean ionization energy deposit [360]. With HiRes being a fluorescence detector, the energy reconstruction is closely related to properties of the atmosphere which is serving as calorimeter. At the same time, atmospheric properties also determine the aperture of the detector. The HiRes flux measurements (HiRes I and HiRes II mono) are found to have similar systematic uncertainties [361, 362, 291]. The main contributions to the systematic uncertainty of the energy scale are the absolute calibration of the PMTs (10\%), the limited knowledge of the air fluorescence yield (6\%), and atmospheric conditions (9\%). About $10 \%$ uncertainty results from the rescaling of the measured calorimetric energy to obtain the total shower energy [360], see also Sec.2. Adding the individual contributions in quadrature, the overall systematic uncertainty of the energy reconstruction amounts to $17 \%$ 362.

In contrast to surface arrays, the aperture of fluorescence detectors has to be determined by simulations. Sources of uncertainty are here varying atmospheric conditions, simulation of shower profiles and detector trigger thresholds, and the primary cosmic ray composition. The uncertainty due to varying atmospheric conditions, mainly that of the vertical aerosol optical depth (VAOD), has been estimated to contribute to the aperture uncertainty $15 \%$ [361, 362. In a recent study the other, simulation-related sources of uncertainty were found not to contribute significantly to the overall flux uncertainty of $30 \%$ [363].

The technique employed in the Auger measurement of the flux combines the advantages of surface detector arrays with that of fluorescence detectors [221]. The surface array operates with almost $100 \%$ duty cycle and the aperture can be calculated in a rather straight-forward way for energies well above the trigger threshold. Fluorescence telescopes allow the direct measurement of the calorimetric shower energy, however, their duty cycle is only about $13 \%$. Using a set of well-reconstructed hybrid events. $5^{5}$ one can calibrate the energy estimator for surface detector data in an almost model-independent way. This is done in a two-step process. First the shower signal at $1000 \mathrm{~m}, S(1000)$, is corrected for attenuation to that of an equivalent shower of $38^{\circ}$ zenith angle, $S_{38}$. To avoid any possible bias from simulations this is done with the constant intensity method by requiring the same number of showers per unit of $\sin ^{2} \theta$. In the second step $S_{38}$ is converted to total shower energy.

The aperture of the Auger detector increased continuously during construction and has an uncertainty of less than $3 \%$. The systematic uncertainty of the energy scale coming from the fluorescence energy measurement is estimated to be $22 \%$. The main contributions to this uncertainty are coming from the uncertainty of the fluorescence yield (14\%), the calibration of the fluorescence telescopes (10\%), and the reconstruction method (10\%) [364, 221]. The described calibration procedure for relating $\mathrm{S}(1000)$ to the primary particle energy leads to an uncertainty of $7 \%$ at $10^{19} \mathrm{eV}$ increasing to $15 \%$ at $10^{20} \mathrm{eV}$.

\section{Composition}

\section{Mean Logarithmic Mass}

At energies below $10^{14} \mathrm{eV}$ the abundance of individual elements has been measured with detectors above the atmosphere. At higher energies this is presently not possible due to the low flux values and the large fluctuations in the development of extensive air showers. Thus, in the past, mostly the mean mass has been investigated. An often-used quantity to characterize the composition is the mean logarithmic mass, defined as $\langle\ln A\rangle=\sum_{i} r_{i} \ln A_{i}, r_{i}$ being the relative fraction of nuclei of mass $A_{i}$. Experimentally, $\langle\ln A\rangle$ is obtained applying two methods: (i) the quantity is proportional to the ratio of the number of electrons and muons registered at ground level $\langle\ln A\rangle \propto \log _{10}\left(N_{e} / N_{\mu}\right)$, see (19) and (ii) $\langle\ln A\rangle$ is proportional to the observed depth of the shower maximum, according to the relation $X_{\max }^{A}=X_{\max }^{p}-X_{R} \ln A$, see (13). Hence, the

\footnotetext{
${ }^{5}$ Events detected with both, the fluorescence telescopes and the surface detectors are called hybrid events.
} 


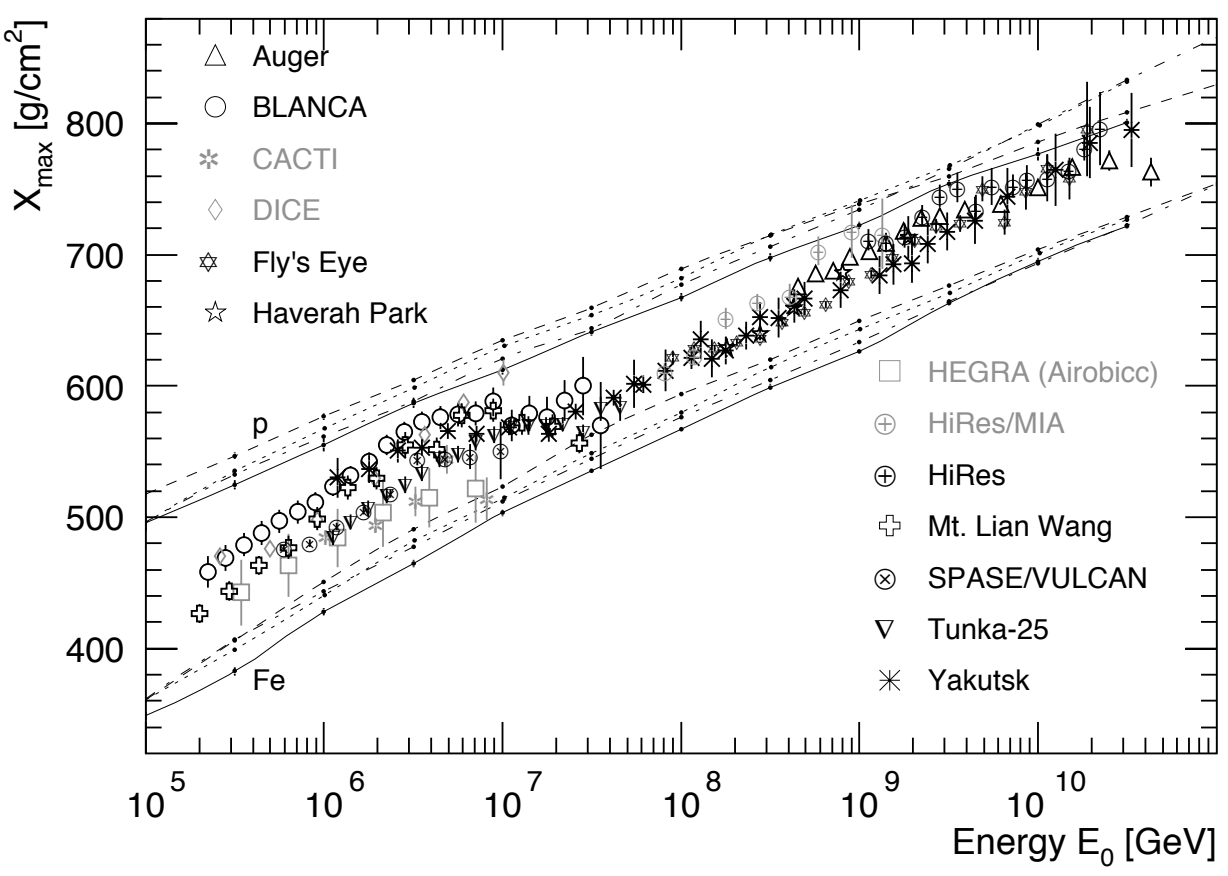

Figure 13: Average depth of the shower maximum $X_{\max }$ as function of primary energy as obtained by Auger [365], BLANCA [225], CACTI [366], DICE [235], Fly's Eye [367], Haverah Park [368], HEGRA [226], HiRes/MIA [290], HiRes [369], Mt. Lian Wang [370], SPASE/VULCAN [371], Tunka-25 [229], Yakutsk [372]. The lines indicate simulations for proton and iron induced showers using the CORSIKA code with the hadronic interaction model QGSJET $01(-)$, QGSJET II-3 (-- ), SIBYLL $2.1(\cdots)$, and EPOS $1.6(-\cdot \cdot)$.

maximum of an iron induced shower should be about $150 \mathrm{~g} / \mathrm{cm}^{2}$ higher up in the atmosphere as compared to a proton induced shower $\left(X_{\max }^{p}\right)$.

Recent measurements of the average depth of the shower maximum are compiled in Fig.13, covering energies from $10^{5}$ to almost $10^{11} \mathrm{GeV}$. The experimental results are compared to predictions of the average depth of the shower maximum from simulations for primary protons and iron nuclei. The CORSIKA code [182] has been used with the hadronic interaction model QGSJET 01 [164, QGSJET II-3 [167], SIBYLL 2.1 [170], and EPOS 1.6 [373]. There are significant differences between the predictions of the different models concerning the absolute values of $X_{\max }$. The differences become important when the model predictions are compared to the experimental data to derive information on the elemental composition of cosmic rays.

Below $4 \times 10^{6} \mathrm{GeV}$ the values obtained by different experiments exhibit a common trend, they seem to increase faster as function of energy than the simulations, which implies that the average composition would become lighter as function of energy. Above the knee $\left(E \gtrsim 4 \times 10^{6} \mathrm{GeV}\right)$ the measured values flatten up to about $4 \times 10^{7} \mathrm{GeV}$, indicating an increase of the average mass in this energy range, as expected from sequential breaks in the energy spectra for individual elements, seen already in Fig.9. Finally, above $4 \times 10^{7} \mathrm{GeV}$ the measured data exhibit about a constant slope for $X_{\max }$ as function of energy. The slope is slightly steeper than the predicted slope for iron nuclei for all models shown. But, on the other hand, a comparison to the predicted proton values is not conclusive, while models like QGSJET 01 favor an extremely light composition at the highest energies, models like DPMJET 2.5 give a hint to an intermediate average mass.

Knowing the average depth of the shower maximum for protons $X_{\max }^{p}$ and iron nuclei $X_{\max }^{F e}$ from simulations, the mean logarithmic mass is derived in the superposition model of air showers 

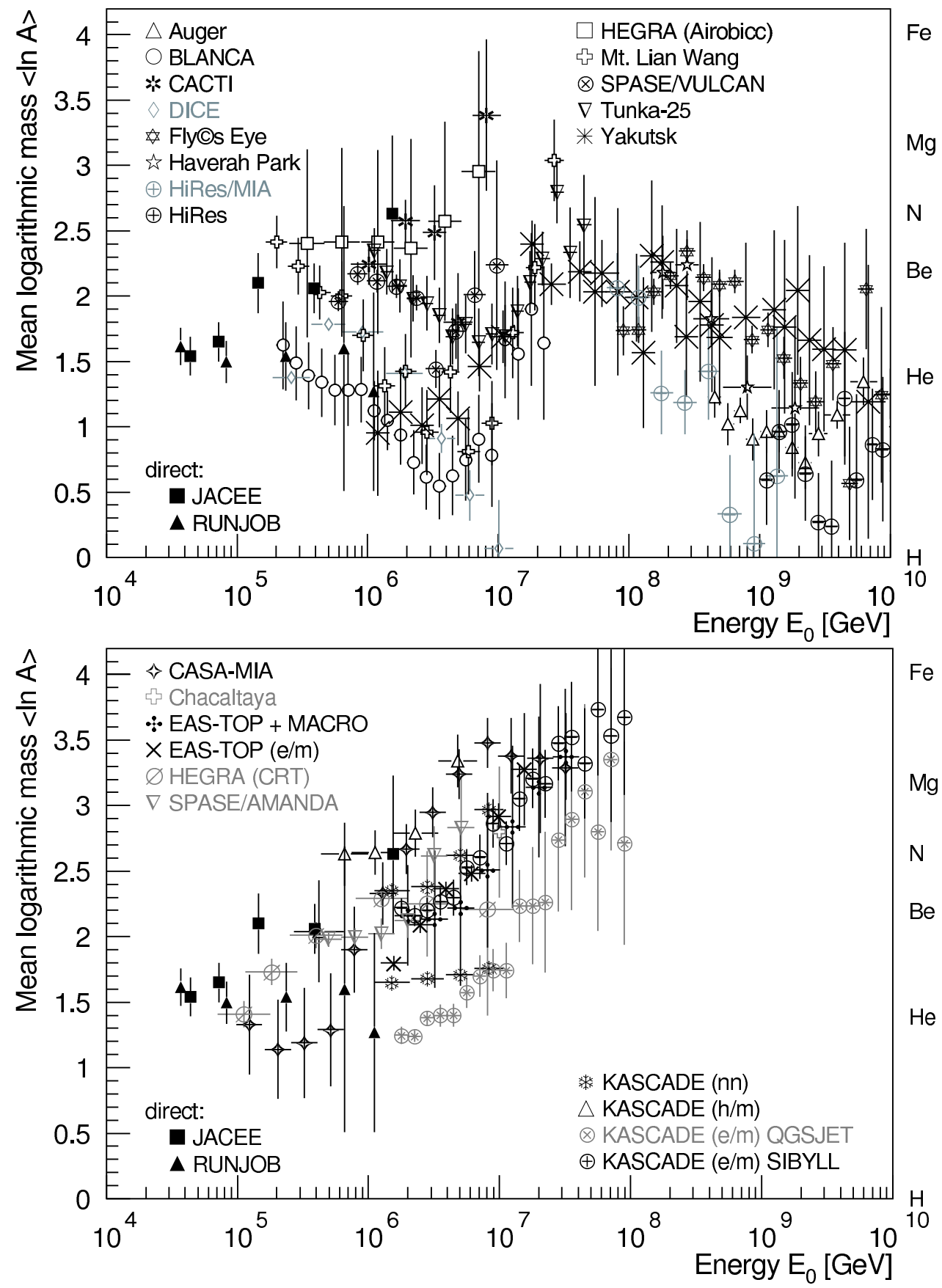

Figure 14: Top panel: Mean logarithmic mass of cosmic rays derived from the average depth of the shower maximum, see Fig.13. The hadronic interaction model QGSJET 01 is used to interpret the measurements. For comparison, results from direct measurements are shown as well from the JACEE [328] and RUNJOB [284] experiments. Bottom panel: Mean logarithmic mass of cosmic rays derived from the measurements of electrons, muons, and hadrons at ground level. Results are shown from CASA-MIA [374], Chacaltaya [375], EAS-TOP electrons and $\mathrm{GeV}$ muons [376], EAS-TOP/MACRO (TeV muons) [377], HEGRA CRT [201], KASCADE electrons and muons interpreted with two hadronic interaction models [286], hadrons and muons [378, as well as an analysis combining different observables with a neural network [297], and SPASE/AMANDA [379]. 
from the measured $X_{\text {max }}^{\text {meas }}$ values using $\langle\ln A\rangle=\left(X_{\max }^{\max }-X_{\max }^{p}\right) /\left(X_{\max }^{F e}-X_{\max }^{p}\right) \cdot \ln A_{F e}$. The corresponding $\langle\ln A\rangle$ values, obtained from the results shown in Fig. 13, are plotted in Fig. 14 (top) as function primary energy using the hadronic interaction model QGSJET 01 to interpret the observed data. For comparison, also results of direct measurements are shown (JACEE and RUNJOB).

In the figure three energy ranges may be distinguished for the indirect measurements. Below about $4 \times 10^{6} \mathrm{GeV}$ the individual experiments seem to indicate a decrease of $\langle\ln A\rangle$ with energy, while above this energy up to about $4 \times 10^{7} \mathrm{GeV}$ an increase with energy is exhibited. At the highest energies $E \gtrsim 4 \times 10^{7} \mathrm{GeV}$, again a decrease with energy can be stated.

Results of measurements of electrons, muons, and hadrons at ground level interpreted with the hadronic interaction code QGSJET 01 are compiled in Fig.14 (bottom). They yield a clear increase of the mean logarithmic mass as function of energy. There seems to be some tension between the results obtained through the observation of the average depth of the shower maximum shown in the top panel and values derived from particle ratios measured at ground level depicted in the bottom panel [3, 380]. In particular, at energies below the knee $\left(E \gtrsim 4 \times 10^{6} \mathrm{GeV}\right)$ the decrease of $\langle\ln A\rangle$ as derived from some $X_{\max }$ measurements is not visible in the particle ratio results.

Using the latest version of QGSJET II does not change the situation qualitatively. The threefold structure of the results obtained is about the same as using QGSJET 01. The main difference are the absolute $\langle\ln A\rangle$ values which are shifted upwards by about 0.8 units for QGSJET II-3 with respect to QGSJET 01. Using the hadronic interaction model SIBYLL 2.1 yields about the same values as for QGSJET II. Using lower inelastic hadronic cross sections in the QGSJET 01 code and larger values for the elasticity of hadronic interactions the discrepancies between $X_{\max }$ measurements and particle ratios at ground can be reduced [380, 381.

\section{Spectra for Elemental Groups}

In addition to the mean mass as discussed above, it is interesting to investigate the energy spectra for individual elements or at least groups of elements.

Information on the flux of primary protons can be inferred from the measurement of the flux of unaccompanied hadrons at ground level. With the KASCADE hadron calorimeter the energy spectrum of single hadrons close to sea level has been measured in the energy range from $100 \mathrm{GeV}$ up to $50 \mathrm{TeV}$ [315]. Based on simulations using the CORSIKA code with the hadronic interaction model QGSJET 01 [164] the energy spectrum of primary hadrons in the energy range from $100 \mathrm{GeV}$ to $1 \mathrm{PeV}$ has been derived. Over the whole four decades in energy it can be described by a single power law.

The KASCADE experiment used the number of electrons and muons $\left(E_{\mu}>230 \mathrm{MeV}\right)$ measured in the scintillator array to reconstruct energy spectra for five primary elemental groups [286. Starting point of the analysis is the correlated frequency distribution of the number of electrons $N_{e}$ and the number of muons $N_{\mu}$. Unfolding algorithms were applied to derive energy spectra for elemental groups. For the analysis the primary particles $\mathrm{H}, \mathrm{He}, \mathrm{C}, \mathrm{Si}$, and Fe were chosen as representatives for five mass groups. Details of the analysis and the used unfolding methods can be found in Ref. [286].

The spectra obtained are presented in Fig.15. To check the influence of the description of hadronic interactions in the atmosphere on the result, the same experimental data were unfolded using two interaction models, namely QGSJET and SIBYLL. The corresponding results are displayed in the figure. The resulting all-particle spectra for both models show a knee at about $4 \mathrm{PeV}$ and coincide within their statistical errors. The decrease of light elements across the knee, i.e. the occurrence of knee-like features in the light element spectra is revealed independently of the used simulation code, as can bee seen in Fig.15. In contrast, the spectra of silicon 

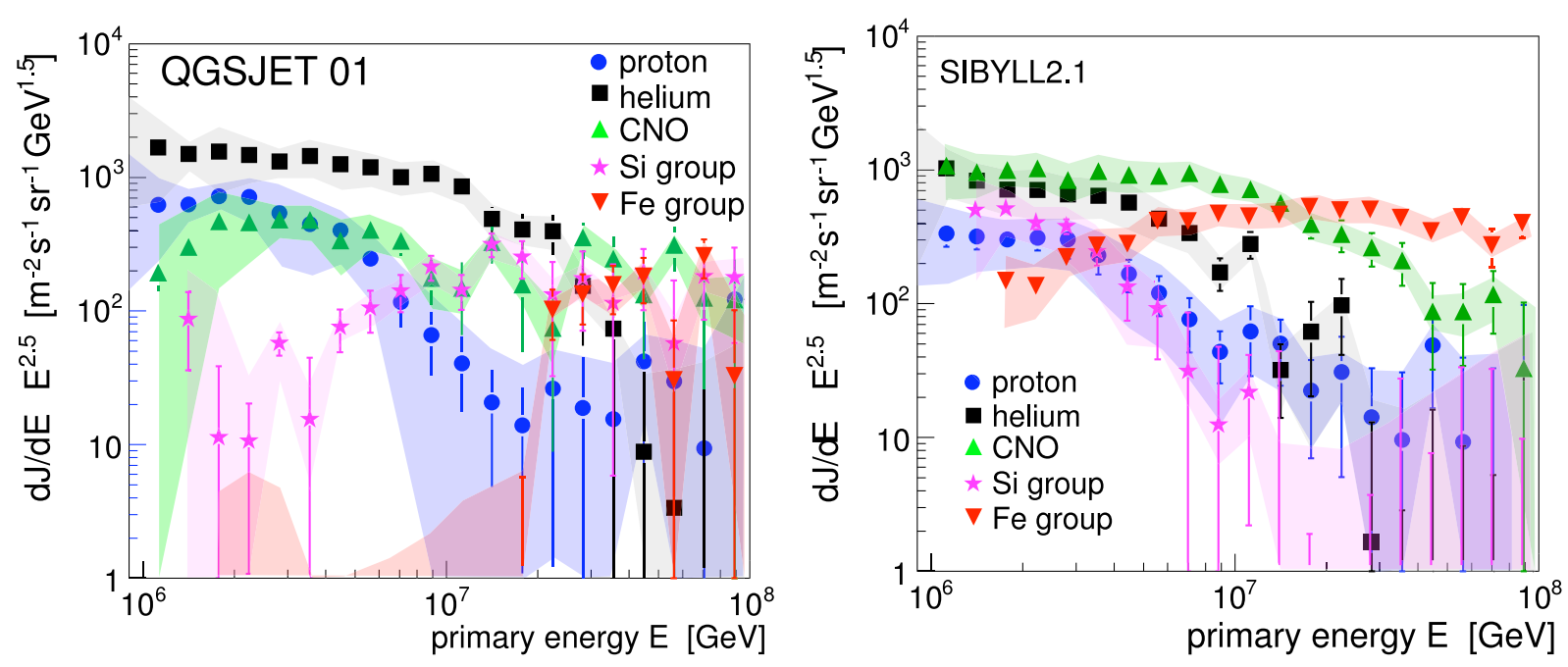

Figure 15: Cosmic-ray energy spectrum for five groups of elements as reconstructed by the KASCADE experiment using the hadronic interaction models QGSJET 01 (left) and SIBYLL 2.1 (right) to interpret the measured data [286].

and iron groups differ significantly and look quite unexpected. This can be understood by judging the ability of the simulations to describe the data. It turns out that both interaction models fail to reproduce the overall correlation between $\log _{10} N_{e}$ and $\log _{10} N_{\mu}$ as observed in the data. In the case of QGSJET simulations the predictions are incompatible with the data in the low energy regime (simulations look too heavy), for SIBYLL incompatibility occurs at higher energies (simulations look too light). Summarizing this analysis, for the first time energy spectra for groups of elements were reconstructed from air shower data. The spectra indicate that the knee in the all-particle spectrum is due to fall-offs in the light element spectra resulting in a heavier composition above the knee.

The EAS-TOP collaboration combined several detection systems to obtain information on the energy spectra of primary cosmic rays. The results are summarized in Fig. 16 .

The proton spectrum in the energy range $0.5-500 \mathrm{TeV}$ [317] has been obtained from the measurement of unaccompanied hadrons with a calorimeter, taking into account the contribution of helium nuclei as obtained by direct measurements [308, 382. The proton spectrum is described over the whole energy range by a single power law.

The helium and CNO fluxes in the energy region from 80 to $200 \mathrm{TeV}$ have been studied from measurements of the Cherenkov light and $\mathrm{TeV}$ muons registered with the underground MACRO experiment 383. Primaries are selected through their energy/nucleon by means of the $\mathrm{TeV}$ muon information. The shower energy is inferred from the measurement of the Cherenkov light yield at distances from 125 to $185 \mathrm{~m}$ from the shower core. The flux for $\mathrm{p}+\mathrm{He}$ at $80 \mathrm{TeV}$ and for $\mathrm{p}+\mathrm{He}+\mathrm{CNO}$ at $250 \mathrm{TeV}$ was obtained. By subtracting the measured proton flux the following values were calculated: $\Phi_{H e}(80 \mathrm{TeV})=(12.7 \pm 4.4) \times 10^{-7} \mathrm{~m}^{-2} \mathrm{sr}^{-1} \mathrm{~s}^{-1} \mathrm{GeV}^{-1}$ and $\Phi_{C N O}(250 \mathrm{TeV})=(0.24 \pm 0.19) \times 10^{-7} \mathrm{~m}^{-2} \mathrm{sr}^{-1} \mathrm{~s}^{-1} \mathrm{GeV}^{-1}$.

The all-particle energy spectrum is obtained from the measured shower size spectra in the knee region [295], showing the angular (i.e. depth) dependence of the knee position. The knee is observed at $N_{e}=10^{6.1}$ in the vertical direction, corresponding to a primary energy $E_{0}=$ $(2-4) \times 10^{15} \mathrm{eV}$, and intensity of $10^{-7} \mathrm{~m}^{-2} \mathrm{~s}^{-1} \mathrm{sr}^{-1}$ with about $20 \%$ uncertainty. The obtained power law indices of the energy spectrum are: $\gamma_{1}=2.76 \pm 0.03$ and $\gamma_{2}=3.19 \pm 0.06$, below and above the knee, respectively.

A composition analysis at knee energies was performed for vertical showers $(1.00<\sec (\Theta)<$ 1.05) through measurements of the number of electrons $N_{e}$ and the number of muons with 


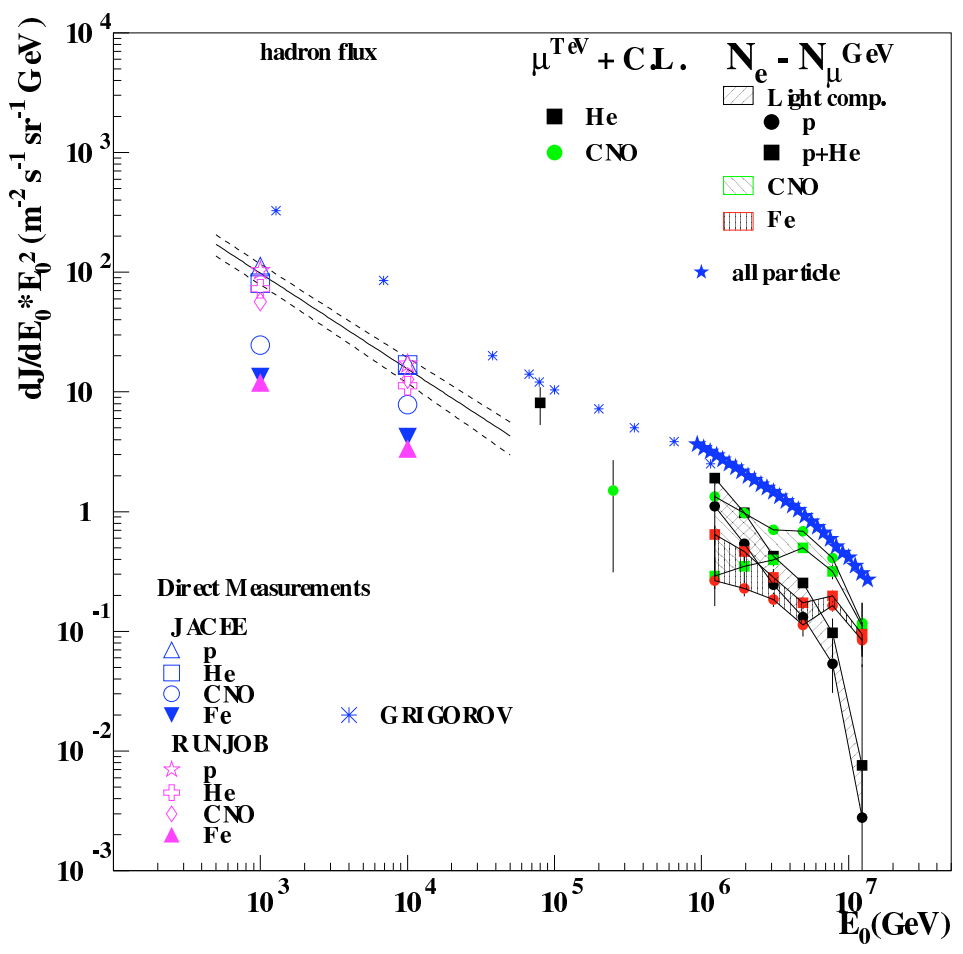

Figure 16: Energy spectrum of cosmic rays as obtained by several detection methods applied in the EAS-TOP experiment [316] compared to results of direct measurements.

energies above $1 \mathrm{GeV}$ recorded in the muon detector at core distances $r=180-210 \mathrm{~m}\left(N_{\mu 180}\right)$. The experimental $N_{\mu 180}$ distributions, measured in different shower size intervals are fitted with simulated data to obtain energy spectra for groups of elements. Intrinsic fluctuations and measurement accuracies allow a three component analysis: light (constructed either with protons, and a mixture of $50 \%$ proton and $50 \%$ helium), intermediate (CNO), and heavy (Fe). The shaded areas in Fig. 16 indicate the energy dependence of the flux thus obtained for the three components.

The Tibet air shower array has the advantage to be located at high altitude $(4300 \mathrm{~m}$, $606 \mathrm{~g} / \mathrm{cm}^{2}$ ). It comprises a scintillation counter array as well as emulsion chambers and burst detectors. The data were used to derive spectra for primary protons and helium nuclei, see Fig.9 [322, 334. However, one has to keep in mind that only a few hundred events remain after quality cuts and are included in the analysis, which may indicate that the results are limited by their statistical significance.

Similar to KASCADE and EAS-TOP, the GRAPES-3 experiment uses the correlation between the registered number of electrons and muons to derive the flux for individual elemental groups. Spectra for protons, helium, as well as the CNO, silicon, and iron groups have been obtained [318], see Fig.9.

\section{Highest Energies}

In 1993 observations with Fly's Eye [40] gave the first indication of a systematic change of the cosmic-ray mass composition at very high energy. Analyzing the mean depth of shower maximum, $\left\langle X_{\max }\right\rangle$, a change from an iron dominated composition at $10^{17} \mathrm{eV}$ to a proton dominated composition at $10^{19.3} \mathrm{eV}$ was found. However, an analysis entirely based on the mean $X_{\max }$ is strongly model dependent (e.g. [384, 158, 385, 386, 380]).

In Fig. 17 (left) a compilation of measurements of $\left\langle X_{\max }\right\rangle$ is shown together with model 

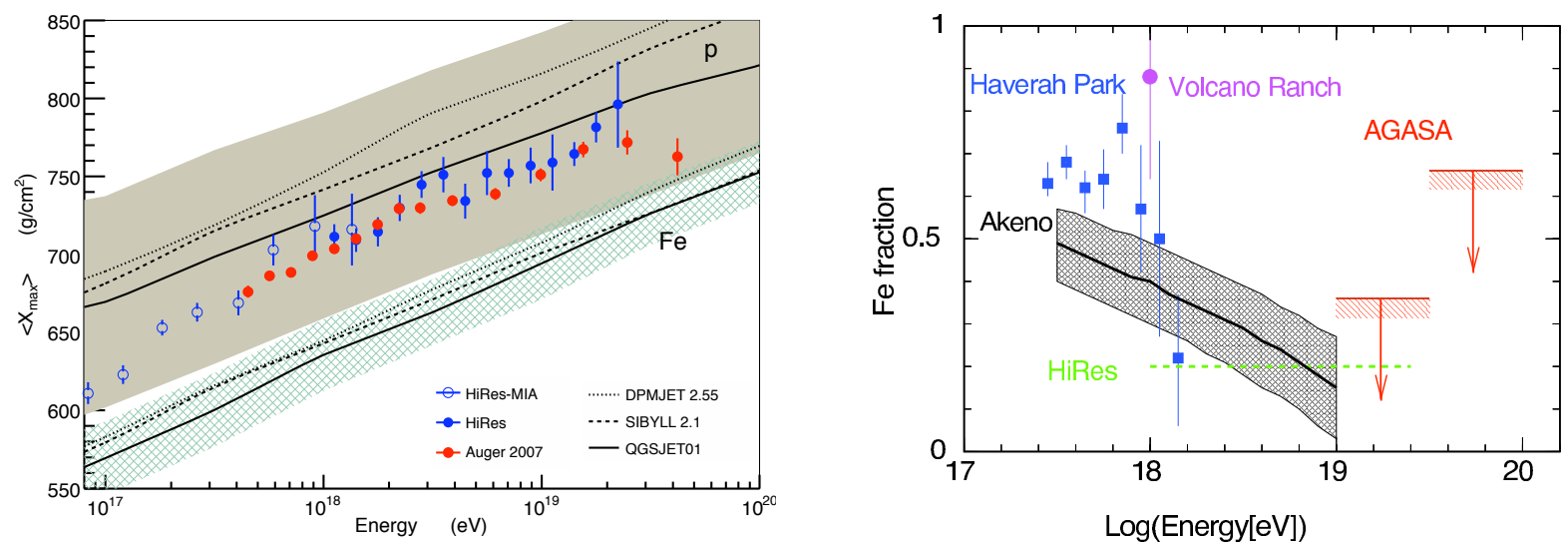

Figure 17: Left panel: Compilation of fluorescence-based measurements of the mean $X_{\text {max }}$ of very high-energy air showers. The data are from Auger [365, HiRes-MIA [289], and HiRes stereo [369]. The model predictions are calculated with CORSIKA [182] and are taken from [386, 185]. The QGSJET predictions on the shower-to-shower fluctuations of the depth of maximum are indicated by the shaded (cross-hatched) area for proton (iron) primaries. Right panel: Fraction of iron, if data are interpreted with a hypothetical bi-modal composition of proton and iron primaries only. Shown are Akeno data [387, as well as results from HiRes [369], Haverah Park [222], and Vulcano Ranch [388, 389]. Upper limits to the iron fraction are obtained from AGASA data 390$]$.

predictions. Adopting the QGSJET 01 model [164] the conclusions of [40] still hold, though a mixed composition is expected at $10^{17} \mathrm{eV}$. On the other hand, on the basis of models like SIBYLL 2.1 [157, 169, 170] or DPMJET 2.55 [160] a much more moderate change of the composition is derived. The model ambiguity of the interpretation of $\left\langle X_{\max }\right\rangle$ can be resolved to some degree by studying the measured distribution of $X_{\max }$ [384, 391].

In contrast to the old measurements of Fly's Eye [40] and Yakutsk [392 the HiRes data indicate a change from an iron-like to a proton dominated composition already at $10^{18} \mathrm{eV}$. The two independent measurements are consistent in the overlap region. The large elongation rate of the low-energy data of $\sim 93 \mathrm{~g} / \mathrm{cm}^{2}$ [290] can only be understood in terms of a change of composition (see Sec.2). Any model with scaling violations will predict a change to a lighter composition [156, 385, 154. Also the muon density measured with the MIA detector [393] in the HiRes-MIA setup indicates a change from a heavy to light composition. The observed muon densities, however, are higher or similar than the expectation for iron primaries and not compatible with medium or light nuclei [290, 289].

The new Auger data on the average depth of shower maximum [365] are, within the systematic uncertainties of about $15 \mathrm{~g} / \mathrm{cm}^{2}$ in good agreement with the published HiRes data. On the other hand, if one would just analyze Auger data, a break in the elongation rate is found at a higher energy, at about $10^{18.35} \mathrm{eV}$. The elongation rate is $71 \pm 5 \mathrm{~g} / \mathrm{cm}^{2}$ below this break and $40 \pm 4 \mathrm{~g} / \mathrm{cm}^{2}$ above [365]. The highest energy point of the Auger data is smaller than expected from a constant elongation rate. It is calculated from 13 observed showers and could indicate a transition to heavier elements as one would expect in case of a rigidity-dependent maximum energy of sources.

The right panel of Fig. 17] shows a compilation of composition measurements based on particle detectors, as published in Ref. [390]. The Akeno result given here is re-scaled according to the predictions of the QGSJET model [359].6]

\footnotetext{
${ }^{6}$ The original composition analysis of Akeno data [387] in the energy range $10^{16.5}-10^{19.5}$ eV appeared to be
} 
The muon density of ultra high-energy showers measured with AGASA was analyzed in Ref. [395]. Comparing the data with model predictions from AIRES [396] and QGSJET 01 the following limits on a two component composition were derived: less than $35 \%$ iron in the energy range $10^{19}-10^{19.5} \mathrm{eV}$ and less than $76 \%$ at higher energy (90\% c.l.) [395, 390].

A re-analysis of Haverah Park data was done in Ref. 222. The authors use the sensitivity of the steepness of the lateral particle distribution to the shower development height, which in turn depends on the depth of shower maximum as composition sensitive observable. It was found that the predictions of CORSIKA with the QGSJET 98 model give a good description of the data if a two-component composition with about $(66 \pm 2) \%$ iron is used in the energy range from $2 \times 10^{17}$ to $10^{18} \mathrm{eV}$. At higher energy (from $10^{18}$ to $2 \times 10^{18} \mathrm{eV}$ ), indications are seen for a transition to a lighter composition (see Fig. 17). This is supported by the number of inclined showers with $E>10^{19} \mathrm{eV}$ that have triggered the Haverah Park array [397]. The data analyzed in [397] agree well with simulations assuming all primary particles are protons, though no mass composition study was done.

On the other hand, a first study of the time structure of Haverah Park showers with zenith angles less than $45^{\circ}$ finds a more iron-dominated composition in the same energy range [223]. Also a re-analysis of Volcano Ranch data similar to the Haverah Park favors a large fraction of iron [398. Using $\sim 370$ showers in the energy range from $5 \times 10^{17}$ to $10^{19} \mathrm{eV}$ a fraction of $\sim 90 \%$ (75\%) iron is found for a two component composition and QGSJET 98 (QGSJET 01).

The discrepancy between muon density-based composition measurements and others based on features of the longitudinal profile underlines the shortcomings of the hadronic interaction models currently available. There seems to be a systematic deficit of muons predicted in simulations in comparison with data [290, 357]. First progress in addressing this problem has been made in [163] by increasing the number of pair-produced baryons in the simulation.

\section{Ultra High-Energy Photons}

Using gamma-ray telescopes, such as MAGIC, H.E.S.S., or VERITAS, photons with energies of about $50 \mathrm{TeV}$ have been observed from several sources in the sky, e.g. [410, 411]. At higher energies various air shower experiments searched for gamma rays. Air showers induced by primary photons develop an almost pure electromagnetic cascade. Experimentally they are identified by their relatively low muon content or their relatively deep shower maximum. Since mostly electromagnetic processes are involved in the shower development, the predictions are more reliable and don't suffer from uncertainties in hadronic interaction models.

Results up to energies of $10^{16} \mathrm{eV}$ are summarized in Fig.18 (left) [401]. There are no indications of a substantial fraction of gamma-rays in the high-energy cosmic-ray flux.

The longitudinal profile of the highest energy event observed by Fly's Eye $(E \sim 3.2 \times$ $10^{20} \mathrm{eV}$ ) 258] has been studied by several groups. Comparing the measured shower profile with Monte Carlo simulations shows that this event is well described by a hadronic primary particle [258, 412, 413]. However, due to the large reconstruction uncertainty of the atmospheric depth of the shower profile, a photon cannot be excluded [413].

The deeply penetrating muon component of inclined showers of hadronic origin is employed in an analysis of Haverah Park data in [414, 397]. Using the primary cosmic-ray flux parametrization of [8] less than $48 \%$ of the observed events above $10^{19} \mathrm{eV}$ can be photons (95\% c.l.). At energies above $4 \times 10^{19} \mathrm{eV}$ this limit is $50 \%$.

Based on the analysis of muons observed in high-energy showers at AGASA the following upper limits were derived in [406, 395]: 34\%, 59\% and 63\% for primary energies above $10^{19}$, $10^{19.25}$, and $10^{19.5} \mathrm{eV}$, respectively (95\% c.l.). A separate analysis of the 6 highest energy events

in contradiction to the Fly's Eye composition interpretation [40, see also the analysis in [394]. 

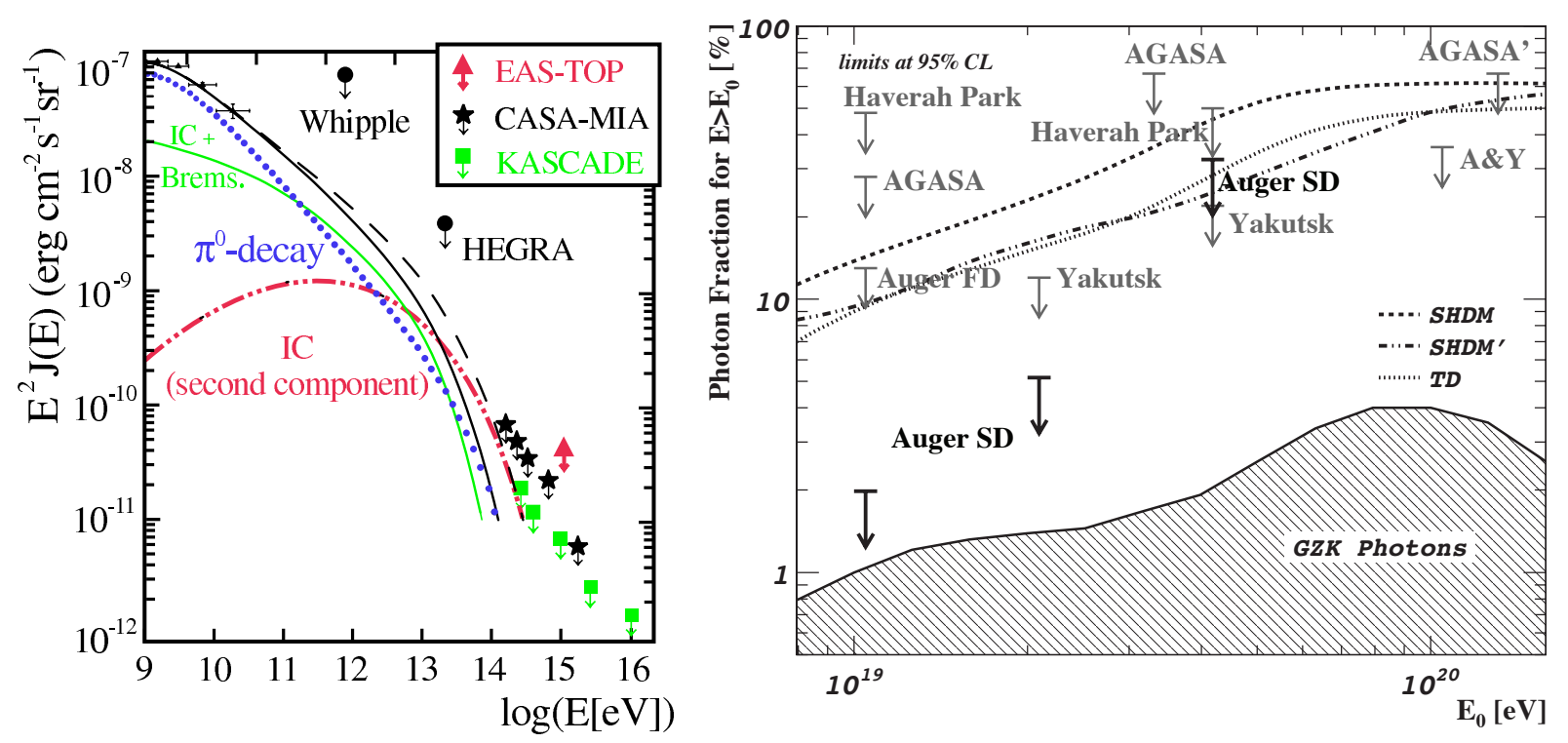

Figure 18: Left panel: Upper limits for the photon flux derived from air shower observations by the CASA-MIA [399], EAS-Top [400, and KASCADE [401] experiments compared to theoretical predictions 402. Right panel: Upper limits on the fraction of photons in the integral cosmic-ray flux compared to predictions for GZK photons and top-down scenarios (SHDM, SHDM', TD) [403. Experimental data are from the Auger surface detectors (Auger SD) [403] and a hybrid analysis (Auger FD) [404, Haverah Park [405, AGASA (marked AGASA and AGASA') [406, 407], AGASA and Yakutsk (A\&Y) [408], as well as Yakutsk [09].

of AGASA was performed in [407]. Using a new method that accounts for the arrival direction of each individual shower, a limit of $67 \%$ at $95 \%$ c.l. could be derived for $E>1.25 \times 10^{20} \mathrm{eV}$.

This new analysis method was also employed in a recent study of Auger shower longitudinal profile data [404. No more than $16 \%$ photons are expected at $95 \%$ c.l. It should be noted that this limit as well as the one derived in [407] are independent of assumptions on hadronic multiparticle production at very high energy. It relies only on the simulation of photon showers which is much better under control than that of hadron-induced showers.

In Ref. [408, the scintillator signals of 10 showers above $10^{20} \mathrm{eV}$ from AGASA and Yakutsk were analyzed to derive a photon fraction limit. The authors report the strongest limit on the photon fraction for showers above $10^{20} \mathrm{eV}$ available so far, less than $36 \%$ photons at $95 \%$ c.l. To obtain this limit, new energies are assigned to the considered showers, partially being very much different from those originally reconstructed. This shows the importance of simulating and understanding detector effects which can only be done in a limited way in such studies. A confirmation by the AGASA and Yakutsk experiments would be very important to establish this limit beyond doubt.

A compilation of recent upper limits on the contribution of photons to the all-particle flux is shown in Fig. 18 [403. The best photon limits are the latest results of the Pierre Auger Observatory [403] setting rather strong limits on the photon flux. They are based on measurements with the Auger surface detectors, taking into account observables sensitive to the longitudinal shower development, the signal rise time, and the curvature of the shower front. The photon fraction is smaller than $2 \%, 5.1 \%$, and $31 \%$ above energies of $10^{19}, 2 \cdot 10^{19}$, and $4 \cdot 10^{19} \mathrm{eV}$, respectively with $95 \%$ confidence level.

In top-down scenarios for high-energy cosmic rays, the particles are decay products of superheavy objects. The decay process yields relatively high fluxes of photons, a typical feature of 


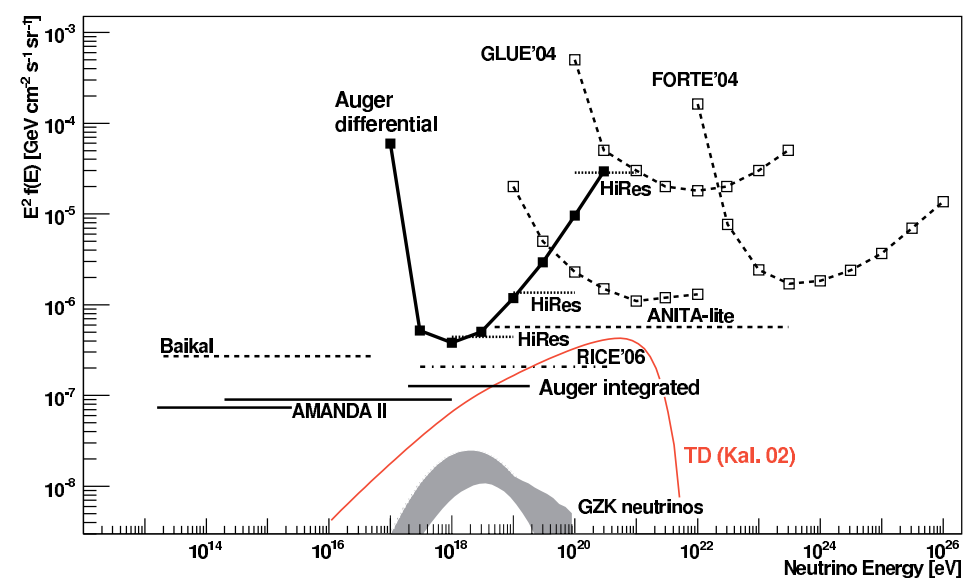

Figure 19: Limits at 90\% confidence level for a diffuse flux of $\nu_{\tau}$ assuming a 1:1:1 ratio of the three neutrino flavors at Earth [416, 13]. The experimental results are compared to predictions for GZK neutrinos and a top-down model [417.

such models [115]. Several predictions are shown in the figure [415, 100]. These scenarios are strongly disfavored by the recent Auger results. It should also be noted that the upper limits are already relatively close to the fluxes expected for photons originating from the GZK effect [124, shown in the figure as shaded area.

\section{Ultra High-Energy Neutrinos}

The detection of ultra high-energy cosmic neutrinos is a long-standing experimental challenge. Many experiments are searching for such neutrinos, and there are several ongoing efforts to construct dedicated experiments to detect them [418, 419]. Their discovery would open a new window to the Universe [126]. However, so far no ultra high-energy neutrinos have been detected. 7

Due to interactions in the source region or during the propagation processes, ultra highenergy cosmic rays are expected to be accompanied by ultra high-energy neutrinos. The neutrinos are produced with different abundances for the individual flavors, e.g. pion decay leads to a ratio $\nu_{e}: \nu_{\mu}=2: 1$. However, due to neutrino oscillations the ratio expected at Earth is $\nu_{\tau}: \nu_{\mu}: \nu_{e}=1: 1: 1$.

To discriminate against the huge hadronic background in air shower detectors, neutrino candidates are identified as nearly horizontal showers with a significant electromagnetic component [425]. The Pierre Auger Observatory is sensitive to Earth-skimming tau neutrinos that interact in the Earth's crust. Tau leptons from $\nu_{\tau}$ charged-current interactions can emerge and decay in the atmosphere to produce a nearly horizontal shower with a significant electromagnetic component. Recent results from the Pierre Auger Observatory together with upper limits from other experiments are presented in Fig. 19. Assuming an $E_{\nu}^{-2}$ differential energy spectrum, the Auger Collaboration derived a limit at $90 \%$ confidence level of $E_{\nu}^{2} \mathrm{~d} N_{\nu_{\tau}} / \mathrm{d} E_{\nu}<1.3 \cdot 10^{-7} \mathrm{GeV} \mathrm{cm} \mathrm{cm}^{-2}$ $\mathrm{s}^{-1} \mathrm{sr}^{-1}$ in the energy range between $2 \cdot 10^{17}$ and $2 \cdot 10^{19} \mathrm{eV}$ [16].

According to top-down models for ultra high-energy cosmic rays a large flux of ultra highenergy neutrinos is expected. As an example, the predictions of a model [417] are shown in the figure as well. This model is disfavored by the recent upper limits. It should also be noted that the current experiments are only about one order of magnitude away from predicted fluxes of GZK neutrinos.

\footnotetext{
${ }^{7}$ Neutrinos produced in air showers (atmospheric neutrinos) 420, in the Sun [21, 422, and during supernova 1987A [23, 424] have been detected, but are at energies well below our focus.
} 


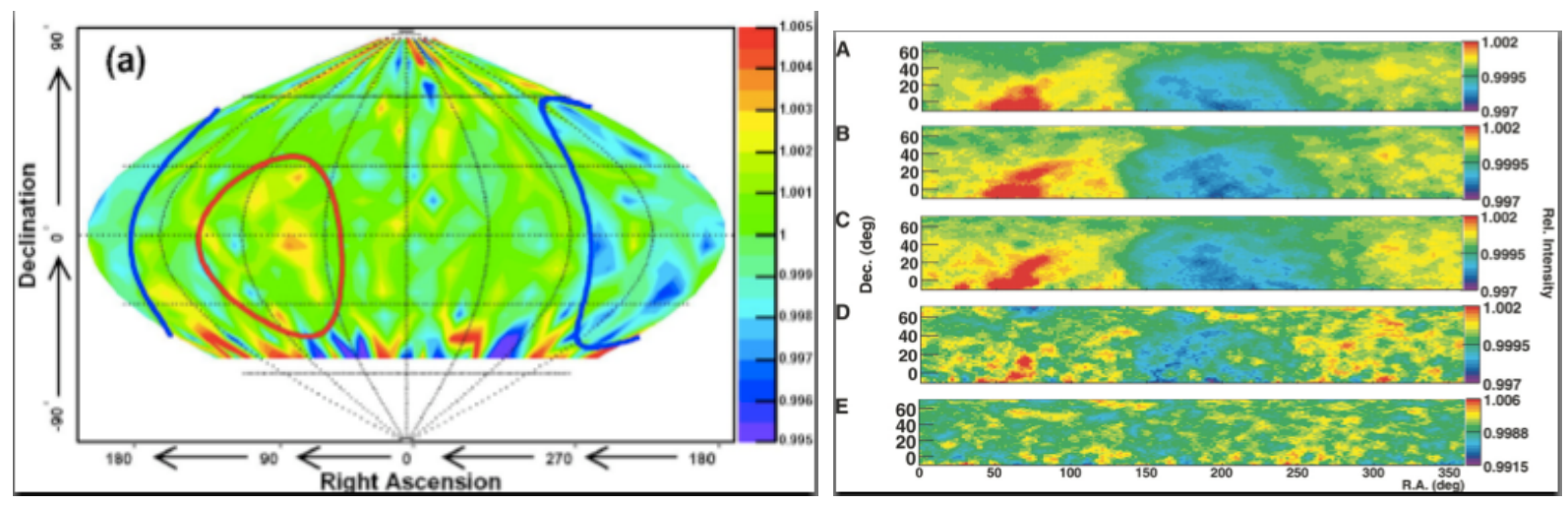

Figure 20: Left panel: arrival direction of cosmic rays in celestial coordinates observed by the Super-Kamiokande experiment. Deviations from the average value for the same declination are shown (amplitude $\pm 5 \%$ ) 438. Right panel: Cosmic-ray intensity map as observed by the Tibet experiment for various energy thresholds, from top to bottom: $4 \mathrm{TeV}, 6.2 \mathrm{TeV}, 12 \mathrm{TeV}, 50 \mathrm{TeV}, 300 \mathrm{TeV}$ 440].

\section{Anisotropy}

The search for anisotropies in the arrival direction of cosmic rays on different angular scales can contribute to the understanding of the cosmic-ray origin, in particular the identification of source regions or individual sources.

\section{Galactic Cosmic Rays}

Large-scale anisotropies are connected to the propagation process of cosmic rays in the Galaxy, while small-scale anisotropies would be a hint towards cosmic-ray sources. However, it has to be considered that the Larmor radius (1) of protons with an energy around $1 \mathrm{PeV}$ in the galactic magnetic field $(B=3 \mu \mathrm{G})$ is of the order of $0.4 \mathrm{pc}$. Hence, it is not expected to find any point sources for galactic cosmic rays. The situation changes for the highest energies, see below.

An excess of charged particles with energies above $10^{15} \mathrm{eV}$ from the direction of a SNR (Monogem ring, $d \approx 300 \mathrm{pc}$ ) has been reported [432] and later withdrawn [433. This supernova remnant with an age of about $10^{5}$ yr has been suggested as possible single source of galactic cosmic rays [434. A signal could not be confirmed neither by the KASCADE experiment [435] investigating cosmic rays with energies above $0.3 \mathrm{PeV}$ nor by the Tibet experiment, looking for PeV $\gamma$-ray emission 436].

The Tibet air shower experiment has performed a northern sky survey, looking for $\mathrm{TeV} \gamma$-ray point sources in a declination range from $0^{\circ}$ to $60^{\circ}$ [437]. A small excess is found, most likely caused by well known $\gamma$-ray sources such as in the Crab Nebula and Mrk 421.

At higher energies, the KASCADE experiment has performed a detailed search for point sources, covering the whole visible sky at energies $E_{0}>0.3 \mathrm{PeV}$ [435]. The visible sky has been divided in cells with a size of $0.5^{\circ}$. Two distributions have been investigated, all events and a selection of muon poor showers. The latter have been investigated in order to look for potential gamma rays. They would manifest themselves in air showers with no or little muons only. Both investigations indicate an isotropic distribution of the arrival direction of cosmic rays. Special attention has been given to the region of the galactic plane, as well as to the vicinity of known SNRs and TeV- $\gamma$-ray sources. No significant excess could be found in either sample.

The Super-Kamiokande experiment investigated large-scale anisotropies for cosmic rays with energies around $10 \mathrm{TeV}$ [338. The experiment registers muons from air showers with an energy threshold of $0.8 \mathrm{TeV}$. An anisotropy map in celestial coordinates is obtained by binning the 
data in pixels of $10^{\circ} \times 10^{\circ}$. The result after applying a smoothing algorithm is shown in Fig. 20 (left). Each cell represents the relative deviation from an isotropic distribution. A $3 \sigma$ excess ("Taurus excess") is found with an amplitude of $1.04 \pm 0.20 \times 10^{-3}$ at right ascension $\alpha=75^{\circ} \pm 7^{\circ}$ and declination $\delta=-5^{\circ} \pm 9^{\circ}$. On the other hand, a deficit ("Virgo deficit") is found with an amplitude of $-(0.94 \pm 0.14) \times 10^{-3}$ at $\alpha=205^{\circ} \pm 7^{\circ}$ and $\delta=5^{\circ} \pm 10^{\circ}$. The angular difference between the Taurus excess and the Virgo deficit amounts to about $130^{\circ}$. A large-scale anisotropy would be expected if the Earth moves relative to a cosmic-ray rest system (Compton Getting effect [41]). For such a scenario the angular difference between maximum and minimum flux should be $180^{\circ}$. Within the relatively large angular uncertainties the anisotropy observed by the Super-Kamiokande experiment would be compatible with a Compton Getting effect caused by a velocity of about $50 \mathrm{~km} / \mathrm{s}$ relative to the rest frame. This velocity is smaller than the rotation speed of the solar system around the galactic center $(\approx 200 \mathrm{~km} / \mathrm{s})$. This implies that the rest frame of cosmic rays (presumably the galactic magnetic fields) is co-rotating with the Galaxy.

The Tibet experiment reported anisotropies in the same regions on the sky [440]. The observed intensity for different energy thresholds is displayed in Fig.20 (right). For energies below $12 \mathrm{TeV}$ the anisotropies show little dependence on energy, whereas above this energy anisotropies fade away, consistent with measurements of the KASCADE experiment in the energy range from 0.7 to $6 \mathrm{PeV}$ [442]. A Compton Getting effect caused by the orbital motion of the solar system around the galactic center would cause an excess at $\alpha \approx 315^{\circ}, \delta=40^{\circ}$ and a minimum at $\alpha=135^{\circ}, \delta=-49^{\circ}$ with an amplitude of $0.35 \%$. However, the measurements at $300 \mathrm{TeV}$ yield an anisotropy amplitude of $0.03 \% \pm 0.03 \%$, consistent with an isotropic cosmic-ray intensity. Hence, a galactic Compton Getting effect can be excluded with a confidence level of about $5 \sigma$. This implies, similar to the result of the Super-Kamiokande experiment, that galactic cosmic rays co-rotate with the local galactic magnetic field environment.

The Tibet experiment finds also an excess with a $0.1 \%$ increase of the cosmic ray intensity in the direction of the Cygnus region $\left(\alpha \approx 309^{\circ}, \delta \approx 38^{\circ} \mathrm{N}\right)$ [40]. In the same region an excess of $\gamma$-rays with energies around $10 \mathrm{TeV}$ is seen by the Milagro experiment [443, 444]. Recently, the Milagro experiment reported also an excess of (charged) cosmic rays with a significance greater than $12 \sigma$ [445]. Presently, different explanations are discussed in the literature: the excess could be related to the Geminga pulsar as local cosmic-ray source [446] or cosmic rays from a local source could reach the Earth via a magnetic mirror [447].

At energies around the knee the Rayleigh formalism has been applied by several groups to characterize the large-scale anisotropy. The two-dimensional distribution of the arrival directions of cosmic rays is reduced to one coordinate because of the limited field of view (typical experiments cover only a fraction of the whole sky) and the small amplitudes expected from theory. A first order approximation of the multipole expansion of the arrival directions of cosmic rays is a harmonic analysis of the right ascension values of extensive air showers.

Applying the Rayleigh formalism to the right ascension distribution of extensive air showers measured by KASCADE yields no hints of anisotropy in the energy range from 0.7 to $6 \mathrm{PeV}$ [442]. This accounts for all showers, as well as for subsets containing showers induced by predominantly light or heavy primary particles. Also other experiments, like Mt. Norikura [448], EAS-TOP [449], Akeno [451, and Adelaide [452] have derived Rayleigh amplitudes. Some experiments find anisotropies, however the phases do not agree between the different results. Hence, it seems to be more likely that all amplitudes derived should be considered as upper limits.

\section{Extragalactic Cosmic Rays}

Different groups have searched for large-scale anisotropies but no confirmed deviation from an isotropic arrival direction distribution has been found. A dipole amplitude that is compatible with full isotropy was found in an analysis of more than 135000 showers with energies from 
$3 \times 10^{16}$ to $10^{17.5} \mathrm{eV}$ of the Yakutsk array [453. Also no significant anisotropy is found in the AGASA data [454] in the energy range between $10^{17}$ to $10^{17.5} \mathrm{eV}$.

In the energy range around $10^{18} \mathrm{eV}$, however, AGASA found an excess of showers coming from directions near the galactic center and the Cygnus region [454]. Furthermore, analysis of SUGAR data [455] indicates an excess of cosmic rays coming from a similar region. The Haverah Park and Yakutsk arrays are located too far north to be able to see the excess regions of AGASA and SUGAR.

With the galactic center being in the field of view of the southern Auger Observatory, it is ideally suited to search for these possible source regions, though the energy threshold for reaching $100 \%$ acceptance of the Auger surface array is just below $10^{18.5} \mathrm{eV}$. Recently, the Auger Collaboration has performed an analysis of their low-energy showers from the direction of the galactic center. In the energy range from $10^{17.9} \mathrm{eV}$ to $10^{18.5} \mathrm{eV}$, no abnormally over-dense regions were found in the neighborhood of the galactic center [456]. There are 506 (413.6) events found (expected) in the AGASA data set for a circle of $20^{\circ}$ around $280^{\circ}$ right ascension and $-17^{\circ}$ declination, corresponding to an excess ratio of 1.2. In the same region, the Auger Collaboration observed 2116 events while 2170 were expected.

At energies above $10^{18.5} \mathrm{eV}$, the large-scale structure of the arrival direction distribution appears, within the limited statistics of the AGASA array, isotropic [454]. This finding agrees with that of the HiRes Collaboration, performing a global anisotropy search based on $\sim 1500$ events observed by HiRes I in monocular mode [457. By combining data from arrays of the northern and southern hemispheres a full sky anisotropy study is done in [458. Considering in total 99 showers from AGASA and SUGAR with $E>10^{19.6}$ no large-scale anisotropy is found. The data set of the Auger Observatory analyzed in [459] contains more than 7000 events with $E>10^{18.3} \mathrm{eV}$. No large scale anisotropy is found. The upper limit to the amplitude of a dipole-like anisotropy in right ascension is 3\% with 95\% CL [459].

Small angle clustering could be an indication for point sources. At energies above $4 \times 10^{19} \mathrm{eV}$, clustering at an angular scale of $2.5^{\circ}$ has been reported by the AGASA Collaboration [460. This result could not be confirmed. An analysis of a HiRes I data set corresponding to an exposure similar to that of AGASA did not reveal any evidence for small scale clustering [463. Also no significant clustering is seen in a data set from HiRes stereo observations of more than 270 showers with $E>10^{19} \mathrm{eV}$ [464] and in the combined AGASA-HiRes stereo data set with $E>4 \times 10^{19} \mathrm{eV}$ [465]. Clustering at the angular scale of the AGASA signal is also not found in the Auger data [466].

Uchihori et al. included showers above $4 \times 10^{19} \mathrm{eV}$ from all four surface arrays of the northern hemisphere in their small scale correlation analysis [467]. The combined data set is found to contain many clusters, however, the statistical significance is low $(\sim 10 \%)$. On the other hand, there are indications for a correlation with the super galactic plane. Restricting the considered arrival directions to the range of $\pm 10^{\circ}$ off the super galactic plane the chance probability for finding doublets and triplets decreases to the order of $1 \%$.

Studies of small angle correlations with the Yakutsk array are difficult because of the angular resolution of the shower axis reconstruction, which is about $4^{\circ}$ [468]. Nevertheless, at much lower energy, clusters of the arrival directions of showers in the energy range $(1.3-4) \times 10^{17} \mathrm{eV}$ were reported in [469]. The direction of these clusters seem to support a correlation with the super galactic plane. Dividing the observed cosmic-ray showers into isotropic and cluster components this correlation can be enhanced significantly [470].

A recent search for correlations at a medium angular scale has been carried out in [471. Publicly available data sets from AGASA, HiRes, SUGAR, and Yakutsk with $E>4 \times 10^{19} \mathrm{eV}$ were combined by adjusting their energy scales to shift the ankle in all data sets to the same energy. In addition events with $E>10^{20} \mathrm{eV}$ from Haverah Park, Volcano Ranch, and Fly's Eye were considered. In this set of 107 events in total, a signal at a scale of $25^{\circ}$ is found in 


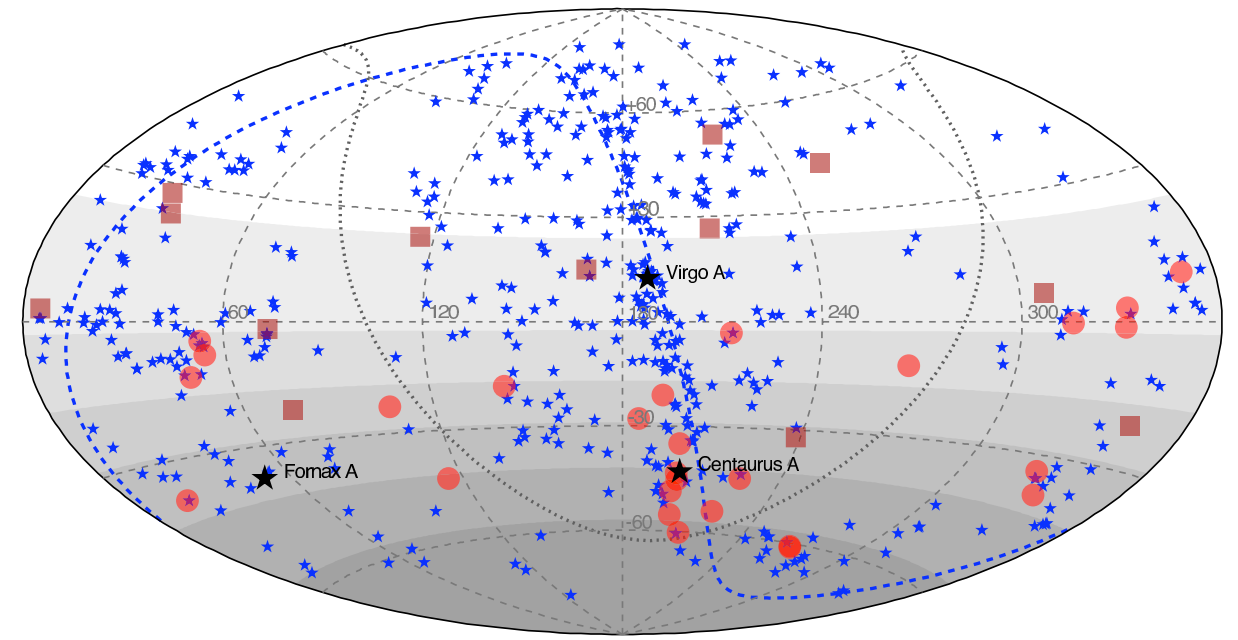

Figure 21: Arrival directions (equatorial coordinates) of the highest energy cosmic rays observed with the Pierre Auger Observatory [484] (circles) and the HiRes telescopes [337. (squares). The asterisks indicate the position of active galactic nuclei (AGN) from the Veron-Cetty Veron catalog [481] up to a distance of $75 \mathrm{Mpc}$. The shaded area indicates the relative exposure of the Auger data set. The dotted line marks the galactic disk and the dashed curve is the super galactic plane.

the autocorrelation function. Results from the Pierre Auger Observatory also indicate some clustering on intermediate scales with an angular separation of order of $15^{\circ}$ to $25^{\circ}$ [466. The medium angle correlation reported in [466] has a $2 \%$ chance probability to originate from an isotropic distribution.

There is a long history of searches for correlations with astrophysical point sources such as colliding galaxies and powerful radio galaxies. It appears almost impossible to assess unambiguously the chance probability of such correlations. First of all, highly incomplete catalogs of astrophysical objects necessarily have to be used in these analyses. Secondly, the penalty factor for scans of several catalogs, selecting different classes of objects, distance and angular ranges, and other parameters cannot be calculated reliably. Part of these problems can be avoided by defining a prescription before analyzing a data set. This has been done by the Auger Collaboration [472].

A correlation with BL Lacertae, at a distance exceeding the GZK energy loss length, has been claimed for the AGASA and Yakutsk high-energy data $\left(E>4 \times 10^{19} \mathrm{eV}\right)$ [473]. The significance of this correlation is highly debated [476] as there might be "hidden" trials involved that cannot be corrected for with a Monte Carlo simulation. These correlations were tested with the independent data set of HiRes stereoscopic observations [480]. None of the previous claims could be confirmed. Recently, a $\sim 0.8^{\circ}$ correlation between BL Lacertae objects of the Veron-Cetty \& Veron catalog [481] with HiRes data $\left(E>10^{19} \mathrm{eV}\right)$ was pointed out [482]. This correlation was confirmed at a nominal significance of about $0.5 \%$ not only for the high-energy part but also for the entire set of HiRes stereo data [480]. An analysis of data from the Pierre Auger Observatory did not confirm a correlation of the arrival direction of cosmic rays with the positions of BL Lacertae objects in the southern hemisphere [483].

A breakthrough in the anisotropy searches is the correlation discovered by the Auger Collaboration [484, 485]. The arrival directions of the very highest energy events $\left(E>5.7 \times 10^{19} \mathrm{eV}\right)$ are found to be correlated with the positions of active galactic nuclei (AGNs) from the Veron-Cetty \& Veron catalog [481]. Out of 27 events observed with an integrated aperture of $9000 \mathrm{~km}^{2} \mathrm{sr} \mathrm{yr}$, 
20 are correlated with AGNs within an angular distance of $3.1^{\circ}$. The correlation was initially found in an exploratory search with different catalogs (12 out of 15 events were correlated). A prescription was set up to verify or reject the correlation hypothesis using an independent data set. Of the next 8 events that were detected, 6 were correlated with AGNs within the prescribed phase space, corresponding to a chance probability of incorrectly accepting the hypothesis of correlation of less than 1\%. After accepting the correlation hypothesis, the Auger group made a scan to refine the correlation parameters and found an energy threshold of $E_{\mathrm{th}}=5.7 \times 10^{19} \mathrm{eV}$, an "source" distance of less than $z=0.017(D \approx 75 \mathrm{Mpc})$ and a maximum angular difference of $3.2^{\circ}$ as optimal parameters. If one does not account for the penalty factor due to different trials and the parameter scans, the nominal chance probability of being compatible with isotropy of $\sim 10^{-10}$ would be obtained.

A sky map of the measured arrival direction distributions is shown in Fig. 21 using equatorial coordinates. The relative exposure of the Auger Observatory is indicated by the shaded regions. The Veron catalog of AGNs is not a complete catalog. As expected, in the direction of the galactic plane the density of detected AGNs is lower than in other directions.

The nearby AGNs are very good tracers of the local matter distribution. In particular, the super galactic plane is clearly visible. A recent update of the super galactic plane position even improves the correlation between AGNs and this matter over-density [486]. The correlation of the arrival direction distribution of UHECRs with nearby AGNs is also reflected by the autocorrelation function which shows some indications of anisotropy in the $15^{\circ}$ to $25^{\circ}$ range, as one would expect from the typical thickness of the super galactic plane.

Given the limited statistics one cannot conclude from the found correlation that AGNs are sources of UHECRs. Subsequent studies of the published highest energy events of the Auger Observatory revealed correlations with the large scale structure of galaxies [487]. A correlation of UHECRs with the large scale structure in the cosmological neighborhood is also found in the Yakutsk data set [490].

The HiRes Collaboration has used the correlation parameters published by Auger to perform a search in their stereo data set. To obtain the same energy threshold as used in the Auger analysis, they scaled their reconstructed energies down by $10 \%$ [337. Using all stereo data an exposure of roughly $4000 \mathrm{~km}^{2} \mathrm{sr} \mathrm{yr}$ is obtained at the highest energy (see Fig. 10). For a total of 13 events two associations with AGNs were found, while 3.2 such correlations are expected for an isotropic arrival direction distribution. No correlation signal is found. Also the autocorrelation function of the highest energy events from HiRes is perfectly in agreement with the expectations for isotropy. The arrival directions of the selected 13 events are shown in Fig. 21 as well. The exposure distribution of the HiRes data set is very similar to that of the Auger Observatory, but North exchanged with South.

The discrepancy between the Auger and HiRes results are currently not understood, but it is clear that a possible difference of the energy scale of the two experiments could lead to such effects. In addition the reconstruction resolution has to be very good to reproduce the very sharp threshold of the correlation found in Auger data. An independent data set of similar size as the published one will allow to test the anisotropy signal.

\section{Astrophysical Interpretation}

\section{Galactic Cosmic Rays and the Knee}

The measurements indicate that the knee in the all-particle energy spectrum is caused by a break in the spectra for the light elements, yielding an increase of the mean mass of cosmic rays in this energy region. Several scenarios are discussed in the literature as possible origin for the knee, see e.g. [4]. In the following, a current astrophysical picture of the origin of high-energy 

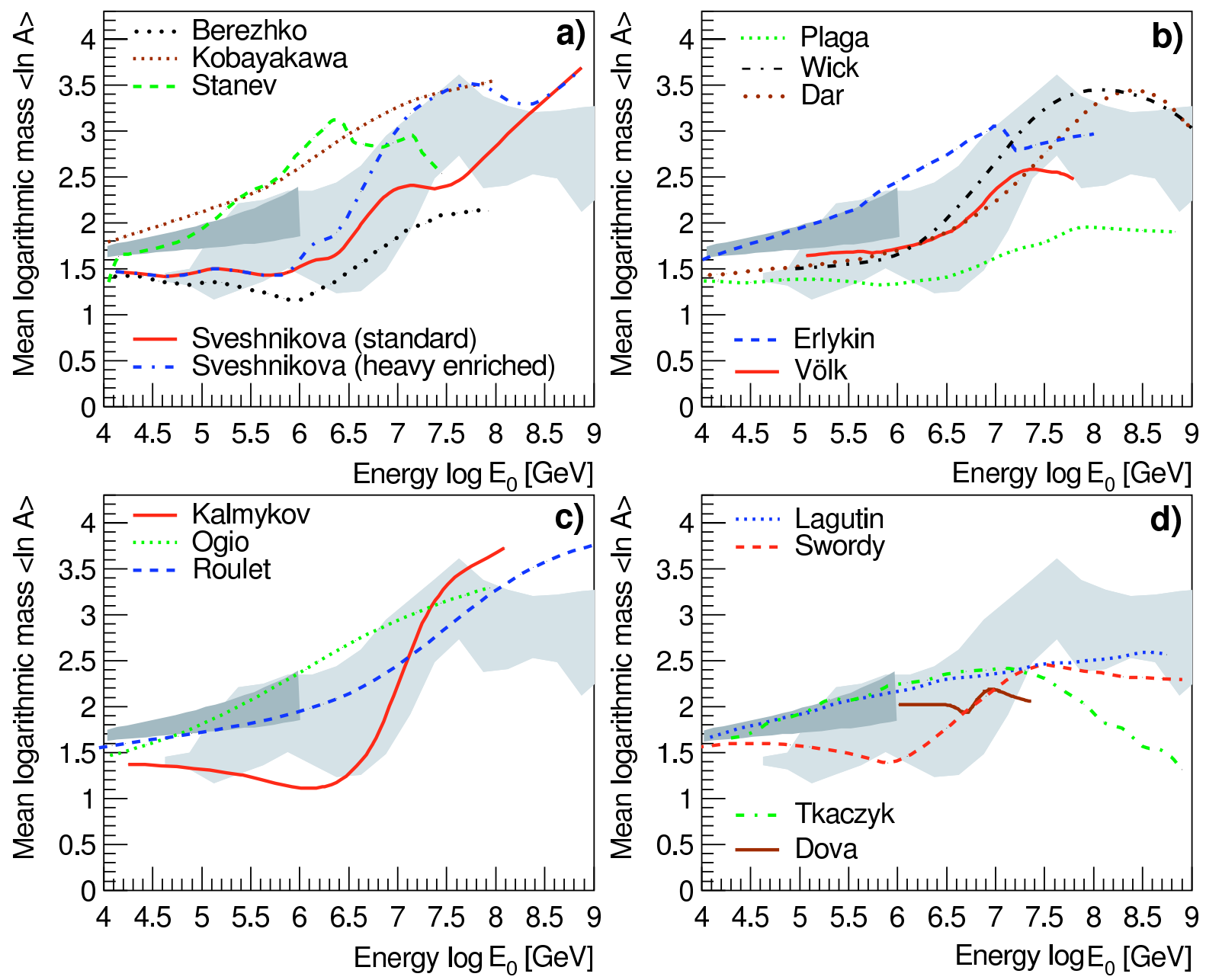

Figure 22: Mean logarithmic mass as function of energy obtained by direct observations (dark gray area) and air shower experiments (light gray area, obtained as weighted average of the results of many experiments, see [4]) compared with different models (lines). a) Acceleration in SNRs (Berezhko et al. [491, Kobayakawa et al. [492, Stanev et al. [493, 494, Sveshnikova et al. [495]); b) acceleration in GRBs (Plaga [496], Wick et al., [497] Dar [498]), single source model (Erlykin \& Wolfendale [499]), reacceleration in the galactic wind (Völk et al. [500]); c) diffusion in the Galaxy (Kalmykov et al. [501, Ogio et al. [502, Roulet et al. [503]); d) propagation in the Galaxy (Lagutin et al. [504], Swordy [505]), as well as interaction with background photons (Tkaczyk [506]) and neutrinos (Dova et al. [507]). For details see [4].

cosmic rays is sketched, based on recent observations.

One of the most popular explanations for the origin of the knee is that the spectra at the source exhibit a break. The bulk of cosmic rays is assumed to be accelerated in strong shock fronts of SNRs [66]. The finite lifetime of a shock front $\left(\sim 10^{5}\right.$ a) limits the maximum energy attainable for particles with charge $Z$ to $E_{\max } \sim Z \cdot(0.1-5) \mathrm{PeV}$. Many versions of this scenario have been discussed [491, 493, 494, 492, 495]. The models differ in assumptions of properties of the SNRs like magnetic field strength, available energy, ambient medium, etc. The differences of the predicted $\langle\ln A\rangle$ can be inferred from Fig. 22 a. While older models [493] limit the maximum energy to about $0.1 \mathrm{PeV}$, recent ideas [495], taking into account latest observations of SNRs, predict maximum energies above $1 \mathrm{PeV}$. In such a model sufficient energy is released from SNRs to explain the observed spectra. A special case of SNR acceleration is the single source 
model [499], which predicts in the knee region pronounced structures in the all-particle energy spectrum, caused by a single SNR. Such structures can not be seen in the compilation of Fig. 8 ,

In the literature also other acceleration mechanisms, like the acceleration of particles in $\gamma$-ray bursts, are discussed [496, 497, 498. They differ in their interpretation of the origin for the knee. The approach by Plaga, assuming Fermi acceleration in a "cannon ball" is not compatible with the measured $\langle\ln A\rangle$ values, see Fig. 22 b. A different interpretation of acceleration in the cannon ball model yields - at the source - a cut-off for individual elements proportional to their mass due to effects of relativistic beaming in jets. The predictions of the actual model are compatible with recent data [498, 508. However, it remains to be clarified how a detailed consideration of the propagation processes, e.g., in a diffusion model, effects the cut-off behavior observed at Earth. Gamma-ray bursts as a special case of supernova explosions are proposed [497] to accelerate cosmic rays from $0.1 \mathrm{PeV}$ up to the highest energies $\left(>10^{20} \mathrm{eV}\right)$. In this approach the propagation of cosmic rays is taken into account and the knee is caused by leakage from the Galaxy leading to a rigidity dependent cut-off behavior.

Also frequently discussed is the idea that the knee is a propagation effect. The propagation is accompanied by leakage of particles from the Galaxy. With increasing energy it becomes more and more difficult to confine the nuclei to the Galaxy. As mentioned above, the path length decreases as $\Lambda \propto E^{-\delta}$. Such a decrease will ultimately lead to a complete loss of the particles, with a rigidity dependent cut-off of the flux for individual elements. Many approaches have been undertaken to describe the propagation process [509, 502, 503, 505, 504]. The Leaky Box model [505] and the anomalous diffusion model [504] yield cut-offs significantly weaker than the data shown in Fig. 9 and [4].

The propagation as described in diffusion models [501, 502, 503] yields $\langle\ln A\rangle$-values which are presented in Fig. 22 c. The models are based on the same principal idea [509], but take into account different assumptions on details of the propagation process, like the structure of galactic magnetic fields etc. This results in a more or less strong cut-off for the flux at the individual knees and, accordingly, in a more or less strong increase of $\langle\ln A\rangle$. The observed break of the spectra is relatively sharp. It seems to be difficult to generate such a behavior by a cut-off at the source or due to propagation only. More likely seems to be a combined approach assuming a break of the spectra at the source and leakage from the Galaxy, as e.g. discussed in [4]. During the propagation phase, reacceleration of particles has been suggested at shock fronts in the galactic wind [500]. Also this mechanism yields a rigidity dependent cut-off.

Another hypothetical explanation for the knee are interactions of cosmic rays with background particles like massive neutrinos [507, 510] or photodisintegration in dense photon fields [506, 511]. Such models appear to be excluded with a high level of confidence. The interactions would produce a large amount of secondary protons, which results in a light mass composition at high energies, not observed by the experiments, see Fig.22 d. Furthermore, a massive neutrino, proposed in [507, 510] can be excluded by measurements of the WMAP and 2dFGRS experiments [512].

A completely different reason for the knee is the idea to transfer energy in nucleon-nucleon interactions into particles, like gravitons [513] or extremely high-energy muons [514], which are not observable (or not yet observed) in air shower experiments. The latter proposal seems to be excluded by measurements of the Baikal experiment [515] setting upper limits for the flux of muons above $10^{5} \mathrm{GeV}$.

No point sources of charged cosmic rays were found in the knee region. The observed (large-scale) anisotropy amplitudes in the energy region of the knee are compared to model predictions in Fig. 23. Two versions of a leaky-box model [516], with and without reacceleration are shown. Leaky-box models, with their extremely steep decrease of the path length as function of energy $\left(\lambda \propto E^{-0.6}\right)$, yield relatively large anisotropies even at modest energies below $10^{6} \mathrm{GeV}$, which seem to be ruled out by the measurements, see also [516, 4]. The measured values are 


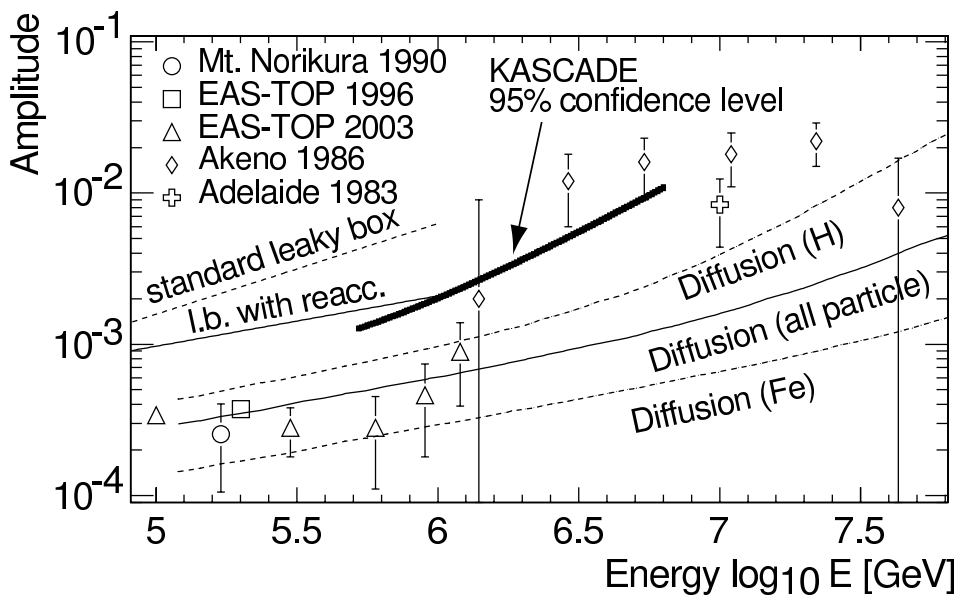

Figure 23: Rayleigh amplitudes as function of energy for various experiments, taken from [442. The results obtained by Mt. Norikura [448], EAS-TOP [449], Akeno [451], Adelaide [452, and KASCADE [442] are compared to predictions of leaky-box models [516] and a diffusion model [517]. For the diffusion model, predictions for primary protons, iron nuclei, and all particles are displayed.

almost an order of magnitude smaller. On the other hand, a diffusion model [517], which is based on the idea of Ref. [509], predicts relatively small values at low energies and a modest rise only. In the figure, predictions for pure protons and iron nuclei are shown together with calculations for a mixed composition. The predicted Rayleigh amplitudes are compatible with the measured values. This may indicate that diffusion models are a realistic description of cosmic-ray propagation in the Galaxy at $\mathrm{PeV}$ energies.

In addition to information extracted from measurements of charged particles, important hints towards the origin of (hadronic) cosmic rays may be derived from observations of high-energy $\gamma$ rays. Recent observation of the H.E.S.S. experiment improve significantly the knowledge about galactic cosmic-ray sources. For the first time a spatially resolved image of a supernova remnant has been obtained with multi-TeV gamma rays as shown in Fig.24 (left) [518]. The shell type supernova remnant RX J1713.7-3946 has been studied in detail [520]. The image of the remnant has been divided into 14 regions to study the energy spectrum of gamma rays up to eight $\mathrm{TeV}$. The indices of the power law spectra vary between $\gamma=1.95 \pm 0.08$ and $2.24 \pm 0.06$ in the different fields with an overall index of the remnant $\gamma=2.13 \pm 0.03$. The spectral indices are very close to the spectral steepness expected from Fermi acceleration at strong shocks. The morphology of the $\mathrm{TeV} \gamma$-ray image agrees well with emissions in the 1-3 keV x-ray regime as measured by the ASCA satellite.

The photon energy spectrum of the supernova remnant RX J1713 is presented in Fig.24 (right). Measurements in various energy ranges (ATCA at radio wavelengths, ASCA x-ray, EGRET GeV $\gamma$-ray, CANGAROO and H.E.S.S. TeV $\gamma$-ray) are compared to predictions of a model by Berezhko \& Völk [519]. The solid line below $10^{6} \mathrm{eV}$ indicates synchrotron emission from electrons ranging from radio frequencies to the x-ray regime. The observed synchrotron flux is used to adjust parameters in the model, which in turn, is used to predict the flux of $\mathrm{TeV}$ $\gamma$-rays. The solid line above $10^{6} \mathrm{eV}$ reflects the spectra of decaying neutral pions, generated in interactions of accelerated hadrons with material in the vicinity of the source (hadron + ISM $\rightarrow \pi^{0} \rightarrow \gamma \gamma$ ). This process is clearly dominant over electromagnetic emission generated by inverse Compton effect and non-thermal bremsstrahlung, as can be inferred from the figure. The results are compatible with a nonlinear kinetic theory of cosmic-ray acceleration in supernova remnants and imply that this supernova remnant is an effective source of nuclear cosmic rays, where about $10 \%$ of the mechanical explosion energy are converted into nuclear cosmic rays 

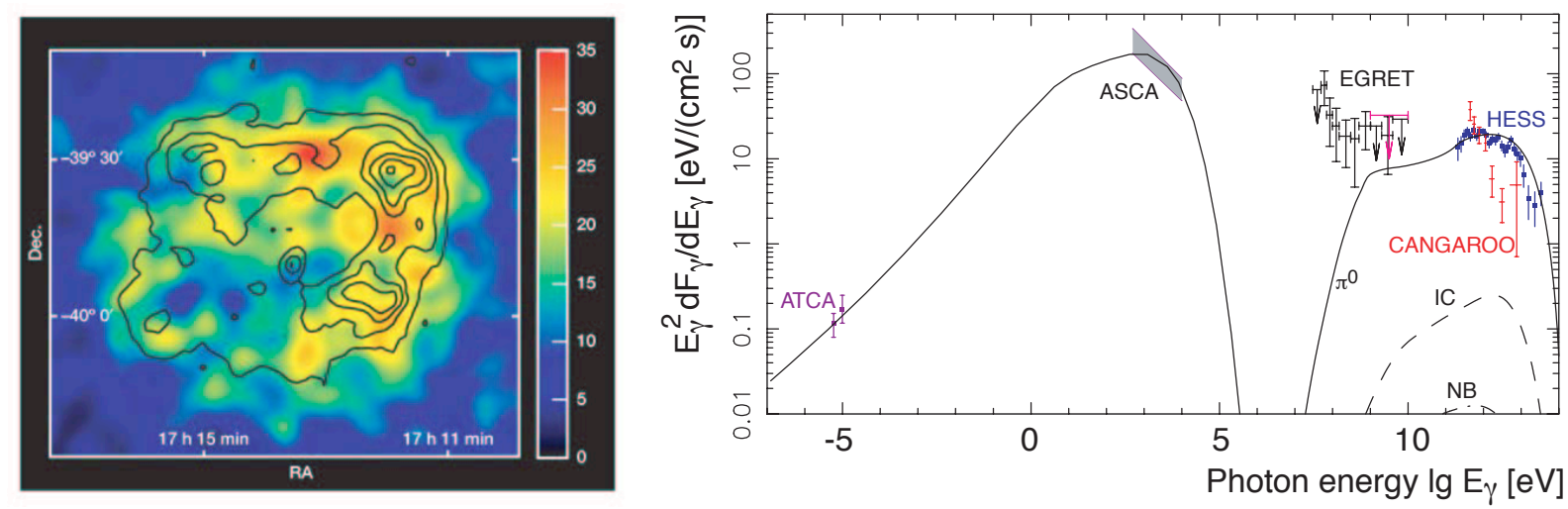

Figure 24: Left panel: $\gamma$-ray image of the supernova remnant RX J1713.7-3946 obtained with the H.E.S.S. telescope. The superimposed contours show the x-ray surface brightness as seen by the ASCA satellite in the 1-3 keV range [518]. Right panel: Spatially integrated spectral energy distribution of the supernova remnant RX J1713. The solid line above $10^{7} \mathrm{eV}$ corresponds to $\gamma$-ray emission from $\pi^{0}$-decay, whereas the dashed and dash-dotted curves indicate the inverse Compton and non-thermal bremsstrahlung emissions, respectively [519].

[519, 521]. Further quantitative evidence for the acceleration of hadrons in supernova remnants is provided by measurements of the HEGRA experiment [522] of $\mathrm{TeV} \gamma$-rays from the SNR Cassiopeia A [523] and by measurements of the H.E.S.S. experiment from the SNR "Vela Junior" 524 .

In conclusion, it may be stated that a standard picture of the origin of galactic cosmic rays seems to emerge from the data. The measurements seem to be compatible with the assumption that (hadronic) cosmic rays are accelerated at strong shock fronts of supernova remnants. The particles propagate in a diffusive process through the Galaxy. As origin for the knee a combination of the maximum energy attained in the acceleration process and leakage from the Galaxy seems to be favored.

\section{Transition Region}

Different scenarios are discussed in the literature for the transition from galactic to extragalactic cosmic rays. The transition most likely occurs at energies between $10^{17}$ and $10^{18} \mathrm{eV}$.

The flux for elemental groups of the model of Hillas is shown in Fig. 25] [525]. The spectra are constructed with rigidity-dependent knee features at high energies. Reviewing the properties of cosmic rays accelerated in SNRs, and using the fluxes as derived by the KASCADE experiment (marked as component $A$ in Fig. 25) Hillas finds that the obtained all-particle flux (dashed line, marked with $Q$ ) is not sufficient to explain the observed all-particle flux, see Fig.25 [525]. Hillas proposes a second (galactic) component to explain the observed flux at energies above $10^{16} \mathrm{eV}$, marked as component "B" in the figure. An extragalactic component, marked as EGT, dominates the all-particle spectrum above $10^{19} \mathrm{eV}$, for details see [525]. Very similar is the model proposed by Wibig and Wolfendale with a transition at higher energies between $10^{18}$ and $10^{19} \mathrm{eV}$ [526]. In this model the galactic cosmic-ray flux extends to higher energies. Thus, a significant contribution of the extragalactic component is required beyond $10^{18} \mathrm{eV}$ only.

Another possibility to match the measured all-particle flux is a significant contribution of ultra-heavy elements (heavier than iron) to the all-particle spectrum at energies around $4 \times$ $10^{17} \mathrm{eV}$ [3, 4], as illustrated in Fig. 26 (left). The figure shows spectra for elemental groups with nuclear charge numbers as indicated, derived from direct and indirect measurements according 


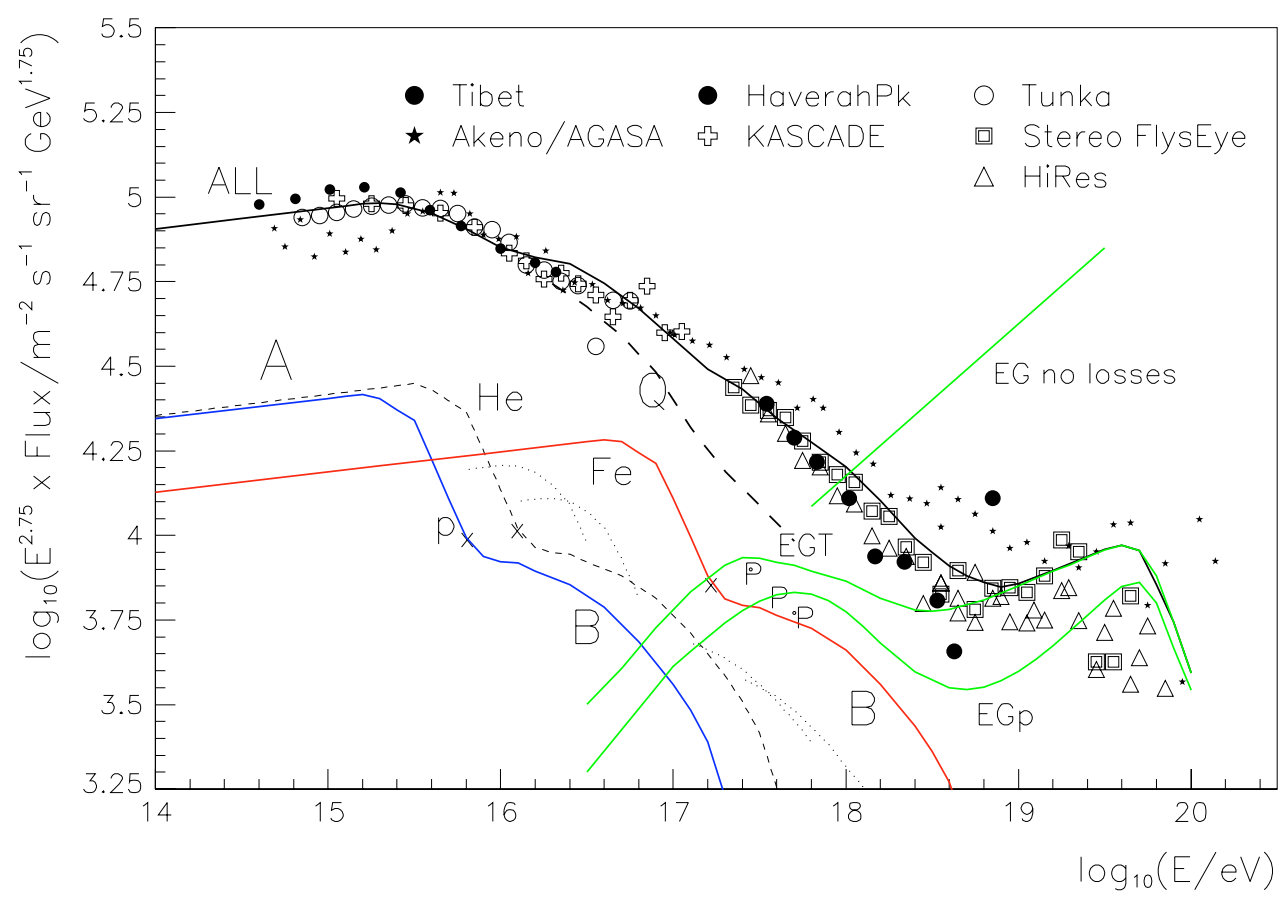

Figure 25: Breakdown of the cosmic-ray spectrum according to a model of Hillas [525] as the sum of galactic $\mathrm{H}, \mathrm{He}, \mathrm{CNO}$, Ne-S, and Fe components with the same rigidity dependence, and extragalactic $\mathrm{H}+$ He having a spectrum $\propto E^{-2.3}$ before suffering losses by cosmic microwave background and starlight interactions. The galactic components were given a turn-down shape based on a KASCADE knee shape as far as the point marked $x$. The dashed line $Q$ is the total galactic SNR flux if the extended tail (component $B$ ) of the galactic flux is omitted. [525].

to the poly-gonato model [3]. The sum of all elements is shown as solid line and is compared to the average experimental all-particle flux in the figure. In this approach the second knee is caused by the fall-off of the heaviest elements with $Z$ up to 92 . It is remarkable that the second knee occurs at $E_{2 n d} \approx 92 \cdot E_{k}$, the latter being the energy of the first knee. In this scenario a significant extragalactic contribution is required at energies $E \gtrsim 4 \times 10^{17} \mathrm{eV}$.

In the model of Berezinsky and collaborators [527, 528], the dip in the all-particle spectrum between $10^{18}$ and $10^{19} \mathrm{eV}$, see Fig. 26 (right), is interpreted as a structure caused by electronpositron pair production on cosmic microwave background photons $p+\gamma_{3 K} \rightarrow p+e^{+}+e^{-}$. Assuming a power law injection spectrum with a spectral index between $\gamma=-2.7$ (without cosmological source evolution) and -2.4 (with cosmological source evolution), the spectrum can be described for $E>10^{17.5} \mathrm{eV}$ with a proton-dominated composition [527]. The shape of the dip is confirmed by data of the Akeno, AGASA, HiRes, Yakutsk, and Fly's Eye detectors after energy-rescaling [528]. Below a characteristic energy $E_{c} \approx 1 \times 10^{18} \mathrm{eV}$ the spectrum flattens and the steeper galactic spectrum becomes dominant at $E<E_{c}$. The transition energy $E_{t r}<E_{c}$ approximately coincides with the position of the second knee $E_{2 n d}$ observed in the all-particle spectrum. The critical energy $E_{c}$ is determined by the energy $E_{e q}=2.3 \times 10^{18} \mathrm{eV}$, where adiabatic and pair-production energy losses are equal. Thus, the position of the second knee is explained in this scenario by proton energy losses on cosmic microwave background photons. The extragalactic component required in the poly-gonato model is somewhere between scenarios 1 and 2 shown in Fig. 26 (right). It should be emphasized that the pair production mechanism requires the primary particles to be dominated $(\gtrsim 80 \%)$ by protons $[345,125]$.

Traditionally, the ankle is interpreted as the characteristic signature for the transition be- 

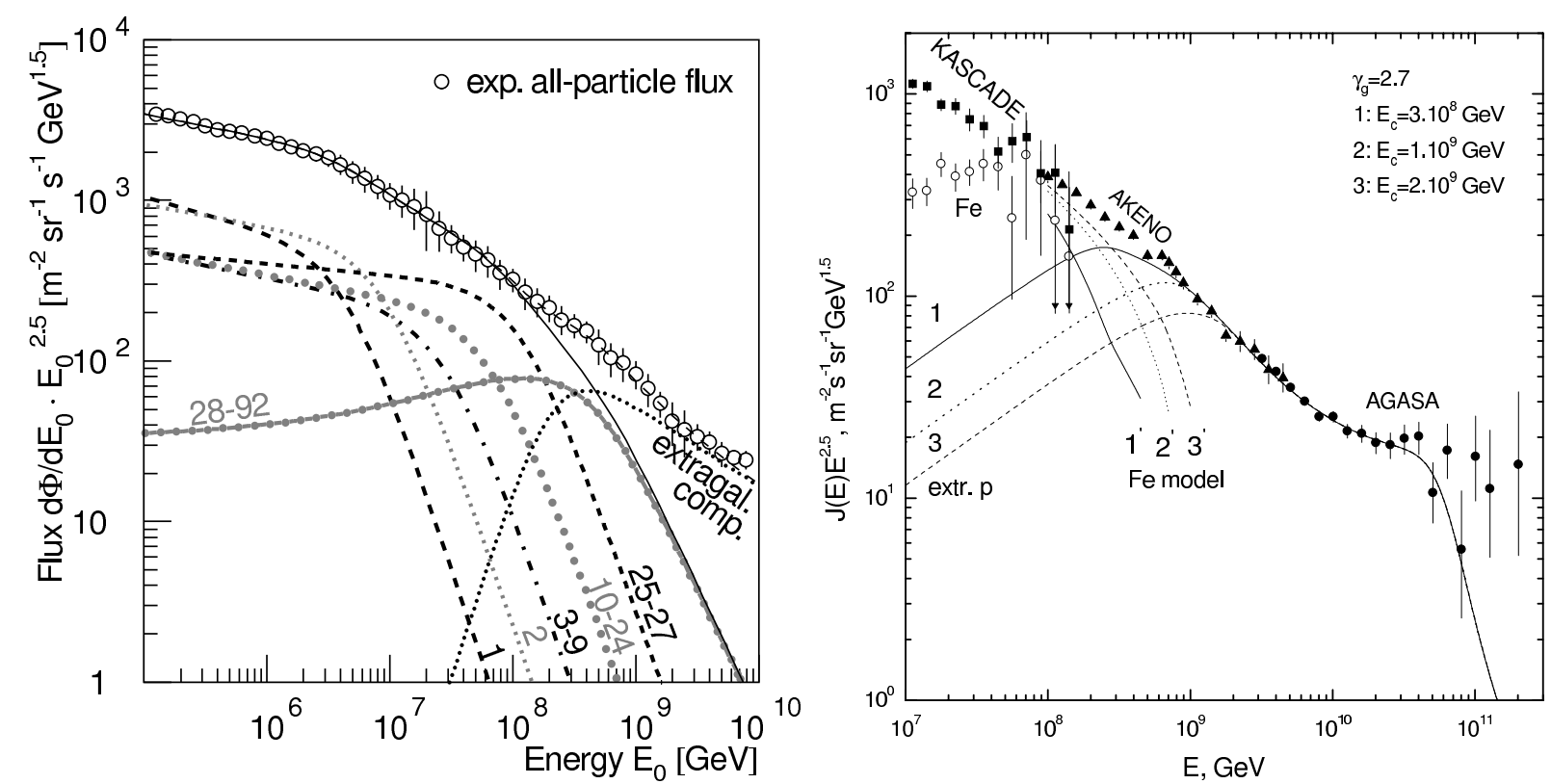

Figure 26: Left panel: Cosmic-ray energy spectra according to the poly-gonato model [3]. The spectra for groups of elements are labeled by their respective nuclear charge numbers. The sum of all elements yields the galactic all-particle spectrum (-) which is compared to the average measured flux. In addition, a hypothetical extragalactic component is shown to account for the observed all-particle flux (---).

Right panel: Transition from galactic to extragalactic cosmic rays according to Berezinsky et al. [527]. Calculated spectra of extragalactic protons (curves 1,2,3) and of galactic iron nuclei (curves 1', 2', 3') are compared with the all-particle spectrum from the Akeno and AGASA experiments. KASCADE data are shown as filled squares for the all-particle flux and as open circles for the flux of iron nuclei.

tween galactic and extragalactic cosmic rays [525, 529]. In such a scenario, extragalactic cosmic rays dominate the flux above about $10^{19} \mathrm{eV}$. This picture of the transition to extragalactic cosmic rays is supported by the pioneering observations of the Fly's Eye experiment that the composition changes at about $10^{18.5} \mathrm{eV}$ [40, 384. New observations by HiRes-MIA and HiRes find a rather sharp transition from a heavy to a light composition at much lower energy, $E \sim 10^{17.5} \mathrm{eV}$. It is clear that the HiRes data are difficult to understand within a model in which naturally heavy elements should dominate the end of the spectrum of galactic cosmic rays just below $10^{19} \mathrm{eV}$.

If one assumes that extragalactic cosmic rays are accelerated in processes qualitatively similar to those in our Galaxy then, at injection, the composition of extragalactic cosmic rays should be similar to that of cosmic rays at lower energy. Indeed, model calculations show that a mixed or even predominantly heavy source composition could, after taking propagation effects into account, be compatible with existing data [125, 530].

On the other hand, the model by Berezinsky et al. predicts a proton-dominated composition at energies as low as $10^{18} \mathrm{eV}$. One of the advantages of this model is the natural explanation of the energy and the shape of the ankle. To obtain a good description of the ankle, there should not be more than $\sim 20 \%$ He in the extragalactic cosmic-ray flux [125, 345]. This could be interpreted as indication for either strong magnetic fields in the accelerating shock fronts or top-down source scenarios, which predict proton-dominated fluxes at not too high an energy.

Understanding the nature of the ankle in the cosmic-ray spectrum has direct implications on the spectrum at much higher energy. For example, if the $e^{+} e^{-}$pair production model is 
confirmed one can conclude that (i) extragalactic cosmic rays are mainly protons, (ii) sources are cosmologically distributed, (iii) there should be a GZK suppression of the flux, (iv) an arrival direction correlation with local sources can be expected, and (v) the injection spectrum of extragalactic cosmic rays is rather steep $\left(d N / d E \sim E^{-\gamma} ; \gamma>2.4\right)$.

Finally it should be noted that the neutrino flux contains complementary information for distinguishing between different model scenarios for the ankle [123, 86, 531].

\section{Extragalactic Cosmic Rays}

Many authors assume extragalactic particles to be nuclei of intermediate to light mass. The discrepancy of elemental compositions derived from mean depth of shower maximum and electron/muon number measurements, however, makes it impossible to use currently available composition measurements as reliable criteria to disfavor models (see also discussion in [533]). This is most strikingly seen in the prototype HiRes-MIA measurements [290, 289]. Whereas the mean depth of maximum data clearly showed the transition to a proton-dominated composition (QGSJET-based interpretation), the muon density at $600 \mathrm{~m}$ - of the same showers - appeared to correspond to primaries as heavy or even heavier than iron. A pure iron composition is obviously not compatible with data. The rather wide distribution of $X_{\max }$ cannot be described with iron primaries only.

The measurements of the HiRes experiment and the Pierre Auger Observatory have given evidence for a suppression of the flux at energies exceeding $4 \cdot 10^{19} \mathrm{eV}[291,221$. The question arises whether this steepening is due to the GZK effect, due to the maximum energy achieved during the acceleration processes, or due to both, a GZK energy loss process and an upper energy limit in the sources.

The support for the existence of the GZK effect is provided by the correlation of the arrival directions with AGN, which imply a strong anisotropy of the arrival direction distribution. The anisotropy appears sharply at an energy of $57 \mathrm{EeV}$. At this energy, the flux measured by the Pierre Auger Observatory is about 50\% lower than expected from a power law extrapolation from lower energies. Thus, there seems to be a connection between the steepening in the spectrum and the AGN correlation. However, if the observed suppression is due to the GZK effect one would expect either a light or rather heavy elemental composition above the GZK threshold. Intermediate mass nuclei are expected to break up very rapidly in interactions with the photons of the 3-K microwave background. The relative abundance of secondary protons produced during propagation according to a recent model [534] is displayed in Fig.27 (right). Observations of the average depth of the shower maximum at the highest energies indicate a mixed composition, see e.g. Fig.13.

The expected flux at Earth according to a propagation model of highest energy cosmic rays by Allard et al. [534] is presented in Fig.27 (left). In this article different scenarios for the properties of the sources are discussed, like different elemental compositions and different spectral indices for the energy spectra at the sources. Figure 27 shows the result assuming a mixed elemental composition at the sources. The contributions of individual elemental groups to the all-particle spectrum are shown. The shape of the energy spectrum measured by the Pierre Auger Observatory is well represented by the all-particle spectrum derived from the model.

Another important source of information are fluxes of particles produced during the propagation of cosmic rays in the Universe. 8 Fluxes of neutrinos and gamma-rays due to the interaction of ultra high-energy cosmic rays with the background photon radiation provide complementary information for discriminating models of UHECRs [122, 123, 535, 536. In Fig. 19 measured

\footnotetext{
${ }^{8}$ In the literature such particles are frequently called "secondary particles". They should not be confused with secondary particles generated in the Earth's atmosphere.
} 

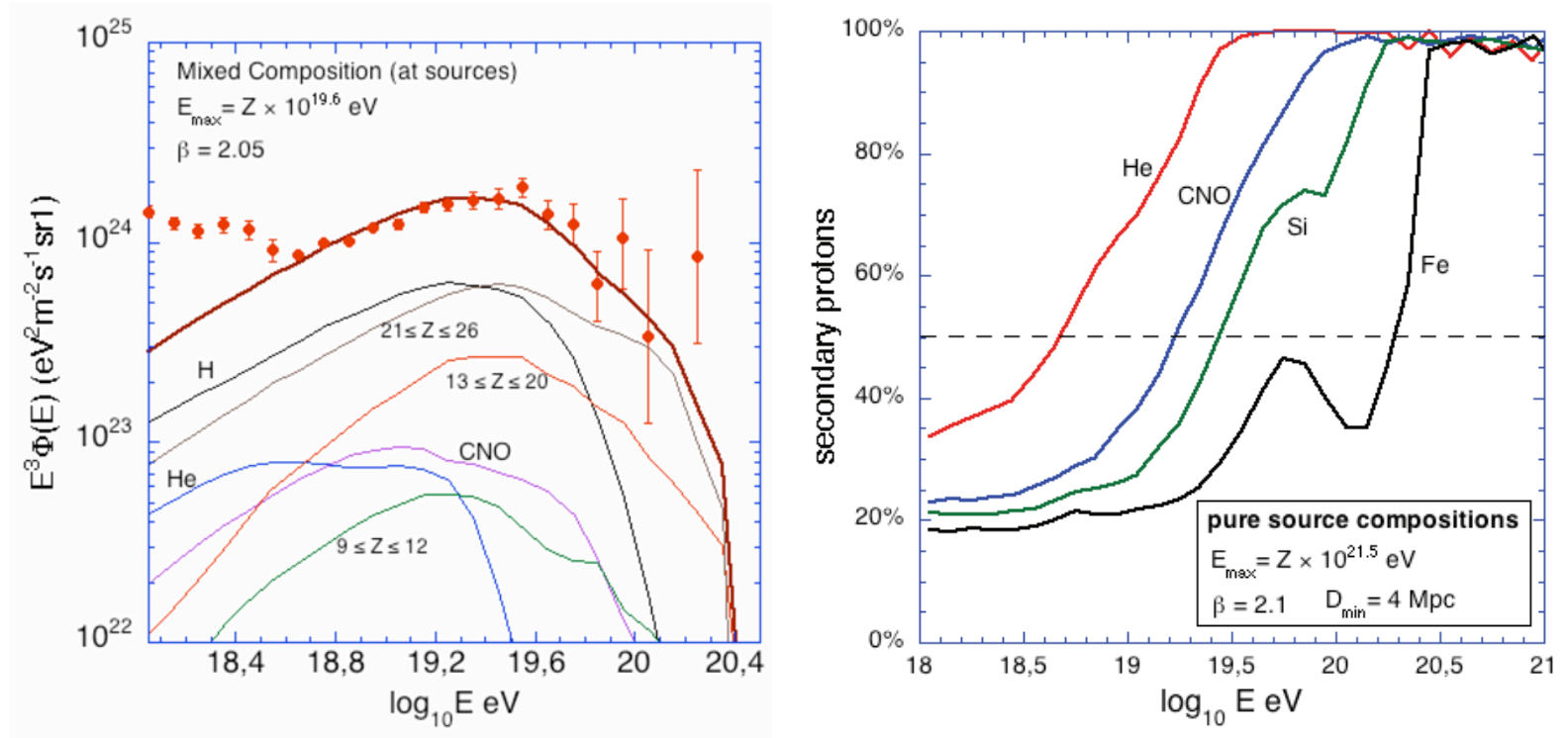

Figure 27: Results of a propagation model of highest energy cosmic rays by Allard et al. [534]. Left panel: Auger data compared to propagated spectra obtained, assuming a mixed source composition. The contributions of spectra for groups of elements to the all-particle spectrum are shown. Right panel: Expected relative abundance of secondary protons as function of energy for different pure source composition hypotheses, assuming a spectral index of -2.1 at the sources.

upper limits for the neutrino flux have been compared to a top-down model [417]. The latest Auger data disfavor this model.

Photons of ultra high energy are easier to detect but their energy loss distance is very short. Nevertheless the measurement of the photon fraction in the primary cosmic-ray flux is one of the most promising methods of distinguishing between different source scenarios of extragalactic cosmic rays [20, 115]. Recent experimental upper limits strongly disfavor predictions of top-down models [415, 100], see Fig. 18 (right). Due to the down-cascading of photons in the extragalactic radiation background, fluxes of $\mathrm{GeV}$ photons are also a complementary source of information. For example, neutrino and photon fluxes of a Z-burst model are discussed in Ref. [122]. Both, the neutrino flux limit by the FORTE satellite [537] and the EGRET diffuse extragalactic photon flux analysis [538] severely constrain this model. Recently, also even more stringent limits were set by the ANITA experiment [539], ruling out most of the parameter space of the Z-burst model. In summary, top-down models are disfavored by recent experimental results of various experiments.

One of the key questions in the field of high-energy astroparticle physics is the understanding of the observed anisotropy above the GZK energy threshold.

The angular scale of about $3^{\circ}$ would favour protons if the AGNs within this correlation angle are indeed the sources of UHECRs [485]. This would be at variance with the Auger data on the mean depths of shower maximum. Most likely, the AGN correlation has to be interpreted merely as a signature of anisotropy and correlation with the nearby matter distribution [540]. First of all, the rate of misidentification of a potential source along the line of sight of the arrival direction of a cosmic ray is very large. Secondly, the AGNs within the correlation window are found to be often less powerful Seyfert 2 galaxies [541]. Thirdly, the AGNs in our cosmological neighborhood are strongly clustered, making it difficult to distinguish between a true AGN correlation and a correlation with larger scale structures.

Instead of assuming a single source in each of the directions of the measured UHECRs, one 
can assign most of the arrival directions to about three sources or source regions [542]. This model scenario would require rather weak galactic magnetic fields and particles in the mass range up to carbon.

In all anisotropy studies even at the highest energies, the galactic magnetic fields play a central role [543]. Knowing their structure to sufficient detail would, for example, allow the determination of the charge of UHECRs. Other interesting applications are the search for the cosmological Compton-Getting effect, a $0.6 \%$ dipole anisotropy that is expected for cosmological sources [544].

A much higher number of UHECRs has to be collected for detailed anisotropy studies of the required sensitivity. If UHECRs are confirmed to be protons, the data can be used for proton astronomy including studies of energy spectra of individual sources and magnetic field spectroscopy, e.g. [116, 545]. This will, of course, require very large-aperture installations as the relevant energy range is just in the GZK suppression region.

\section{Importance of Modeling Hadronic Interactions}

There are strong indications for shortcomings in the shower simulations, probably due to limitations of modeling hadronic interactions.

Detailed studies of the shower development in the atmosphere have been performed with the KASCADE multi-detector set-up and interaction models have been improved [214, 208, 546, 547. A particularly valuable tool to test high-energy interaction models are correlations between different shower components [553, 554]. Some years ago several models like SIBYLL 1.6, DPMJET 2.5, or NEXUS 2 failed to describe the measurements of particular correlations. On the other hand, for modern models like QGSJET 01, SIBYLL 2.1, or DPMJET 2.55, the KASCADE measurements are compatible with predictions for various correlations between the electromagnetic, muonic, and hadronic components, i.e. the measurements are bracketed by the extreme assumptions of primary protons and iron nuclei [553, 554]. In previous analyses pure proton or iron compositions have been assumed as extreme cases. However, at present, more detailed analyses are performed [554, 555]. They take into account the spectra for elemental groups as obtained from investigations of the electromagnetic and muonic components (as discussed above, see Fig. 15). These investigations reveal deviations between measurements and simulations for the hadronic component of the order of $10 \%$ to $20 \%$ [554].

The situation is similar at very high energy. For example, the mean $X_{\max }$ of the HiResMIA data is not consistent with the measured muon densities of the same events [290]. The conclusions on mass composition from Haverah Park data are different if the time structure of the shower front is used instead of the muon yield that determines the rate of inclined showers. The Auger data indicate that the energy scale derived from surface detector simulation seems to be of the order of $25 \%$ higher than that obtained from fluorescence measurements [357.

In the foreseeable future soft multi-particle production will not be calculable within QCD. Therefore the modeling of cosmic-ray interactions will continue to depend strongly on the input from accelerator experiments that is needed to tune phenomenological particle production models. Measurements at both, fixed target and collider experiments can substantially contribute to reducing the uncertainties of the models and, hence, determine the composition of cosmic rays.

High-energy interactions are very important for the overall shower profile but hadronic multiparticle production is least known in this energy range. This is illustrated in Fig. 28 in which predictions of hadronic interaction models are shown for proton-proton collisions at $\sqrt{s}=14 \mathrm{TeV}$. The acceptance ranges for different detector components of the CERN CMS [556] detector for the LHC [557] are also shown.

Every ultra high-energy air shower contains many sub-showers of lower energy. For example, the slope of the lateral distribution of particles in a shower is a measure of the mass of the primary 

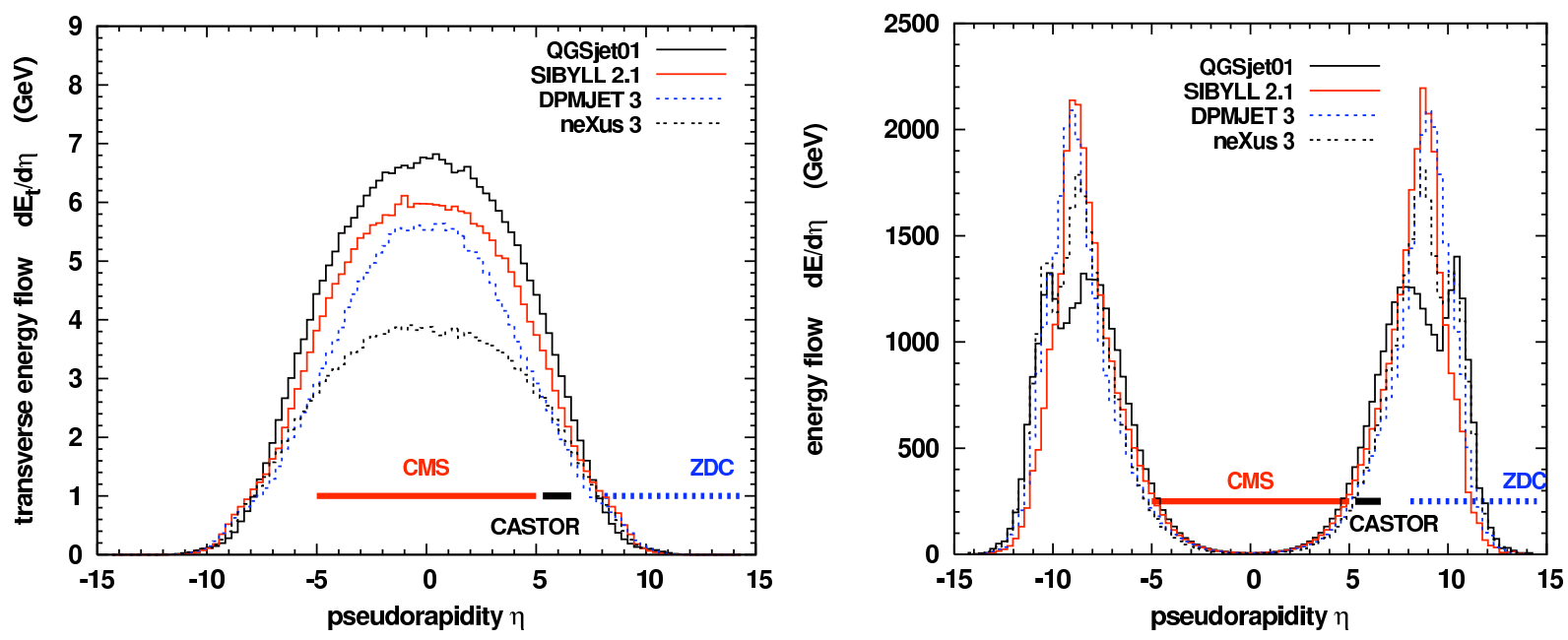

Figure 28: Prediction of the transverse (left) and total (right) energy flow produced in proton-proton collisions at LHC as obtained with cosmic-ray interaction models. Also shown are the acceptance ranges of the CMS central calorimeter, the CASTOR calorimeter and the zero degree calorimeter for neutral particles (ZDC).

particle. Even for high-energy showers of $10^{19} \mathrm{eV}$, this slope is very sensitive to assumptions on hadronic multiparticle production in the energy range of ten to a few hundred GeV [177. The energy distribution of hadronic interactions, in which at least one meson was produced that in turn decayed to a muon that reached sea level, has a maximum in the range between 80 and $200 \mathrm{GeV}$. Most of the interactions are induced by pions (70\%) and nucleons (20\%).

In addition to accelerator measurements of hadronic multiparticle production [558, measurements and understanding of air shower data at lower energy are very important to tune and validate the used hadronic interaction models [560].

\section{Conclusions and Outlook}

The all-particle flux of cosmic rays is reasonably well known up to the highest energies. Recent measurements by the HiRes and Auger Collaborations established a GZK-like suppression of the flux at energies exceeding $6 \cdot 10^{19} \mathrm{eV}$.

In the knee region the mean mass of cosmic rays is found to increase as function of energy. The knee is caused by sequential breaks in the spectra of individual elements, starting with the light elements. At present, a rigidity dependence of the cut-off energies for the individual elements is likely but not yet clear beyond doubt. Above $10^{17} \mathrm{eV}$ the situation becomes very uncertain. Almost no data are available in the important energy range where the galactic cosmic-ray component is expected to end $\left(10^{17}-10^{19} \mathrm{eV}\right)$, at present there are only limited experimental efforts in this region. At the highest energies $\left(\gtrsim 10^{19} \mathrm{eV}\right)$, several experiments indicate a light to mixed composition with a strong dependence on the model used to describe high-energy interactions in the atmosphere.

Large scale anisotropies have been found at low energies, being compatible with the movement of the Earth around the Sun (Compton Getting effect). In the knee region, the anisotropies disappear, indicating that the rest frame of galactic cosmic rays co-rotates with the Galaxy. At ultra-high energies anisotropy measurements provide independent information on the composition due to deflections in the galactic magnetic fields. The results of the Pierre Auger Observatory indicate a correlation between the arrival direction of cosmic rays and the super galactic plane. 
The energy of the transition from galactic to extragalactic particles is discussed controversially. The scenarios considered limit the transition energy to the range between $10^{17}$ and $10^{19} \mathrm{eV}$. Precise composition measurements in this region will be decisive.

The knowledge about the cosmic-ray composition is presently limited by the uncertainties in the hadronic interaction models used to describe the air shower development. The ambiguities can not be resolved by cosmic-ray measurements solely. Collaboration with experiments at the LHC and fixed target experiments is mandatory to improve the understanding of multiparticle production in the (extreme) forward region, thus providing reliable simulation codes.

Independent and complementary information about the origin of high-energy cosmic rays can be obtained by measurements of high-energy gamma rays and neutrinos (multi messenger approach). Recent measurements of $\mathrm{TeV}$ gamma rays from supernova remnants give strong hints for this object class as sources of galactic cosmic rays. Measurements of neutrino and photon fluxes are very useful to distinguish between different scenarios for the transition from galactic to extragalactic cosmic rays. They are important to establish a GZK feature beyond doubt and provide composition information at the highest energies.

In the near future new cosmic-ray detectors will provide additional information on the transition from galactic to extragalactic cosmic rays (KASCADE-Grande [561, IceTop/IceCube [562], extensions of Auger-South [563, 564], extension of the Telescope Array [565]), the anisotropy of the arrival directions and composition of cosmic rays at the highest energies on the whole sky (Auger North [566]) as well as super GZK particles (EUSO [567], a recovery of the cosmic-ray flux beyond the GZK resonance). A promising new detection technique, the measurement of radio emission from air showers, is presently developed in the Pierre Auger Observatory and the LOFAR project. This technique is expected to allow efficient cosmic-ray measurements in future large-aperture experiments.

In foreseeable future no significant improvement of hadronic interaction models is expected. Therefore, the range of direct measurements should be extended to energies approaching the knee. Ideal would be an ACCESS-type [569] experiment in outer space with exposure times of several years. More experimental efforts are needed in the energy region where the galactic component is expected to end. At ultra-high energies the anisotropy studies are limited by statistics only. Therefore, large aperture experiments are needed with full-sky coverage such as the Pierre Auger Project (with observatories in the southern and northern hemisphere).

\section{Acknowledgements}

We thank our colleagues from the Pierre Auger and KASCADE-Grande Collaborations for many fruitful and stimulating discussions and comments on the paper. In particular, we thank Carola Dobrigkeit, Maria Giller and Jim Matthews for reading the manuscript of this article. We are grateful to D. Heck and T. Pierog for providing numerical results of air shower simulations and S. Knurenko, K. Shinozaki, P. Sokolsky, M. Takeda, and G. Thomson for making available data tables of experimental results. This work has been supported in part by BMBF grant 05A08VK1.

\section{References}

[1] B. Wiebel-Sooth and P. L. Biermann, Landolt-Börnstein, New Series vol. VI/3c (1999) 37.

[2] S. P. Swordy et al., Astropart. Phys. 18 (2002) 129-150

[3] J. R. Hörandel, Astropart. Phys. 19 (2003) 193-220

[4] J. R. Hörandel, Astropart. Phys. 21 (2004) 241-265

[5] M. Giller, J. Phys. G35 (2008) 023201. 
[6] C. De Donato and G. A. Medina-Tanco, arXiv:0807.4510 [astro-ph].

[7] P. Sokolsky, P. Sommers, and B. R. Dawson, Phys. Rept. 217 (1992) 225-277.

[8] M. Nagano and A. A. Watson, Rev. Mod. Phys. 72 (2000) 689-732.

[9] X. Bertou, M. Boratav, and A. Letessier-Selvon, Int. J. Mod. Phys. A15 (2000) 2181-2224

[10] R. Engel and H. Klages, Comptes Rendus Physique 5 (2004) 505-518.

[11] J. W. Cronin, Nucl. Phys. Proc. Suppl. 138 (2005) 465-491

[12] D. R. Bergman and J. W. Belz, J. Phys. G Nucl. Part. Phys. 34 (2007) R359-R400

[13] K.-H. Kampert, J. Phys. Conf. Ser. 120 (2008) 062002

[14] A. V. Olinto, Phys. Rept. 333 (2000) 329-348

[15] G. Sigl, Ann. Phys. 303 (2003) 117-141

[16] L. Anchordoqui, T. Paul, S. Reucroft, and J. Swain, Int. J. Mod. Phys. A18 (2003) 2229-2366

[17] R. J. Protheroe and R. W. Clay, Publ. Astron. Soc. Pac. 21 (2004) 1-22

[18] A. M. Hillas, Ann. Rev. Astron. Astrophys. 22 (1984) 425-444.

[19] D. F. Torres and L. A. Anchordoqui, Rept. Prog. Phys. 67 (2004) 1663-1730

[20] P. Bhattacharjee and G. Sigl, Phys. Rept. 327 (2000) 109-247

[21] P. K. F. Grieder, Cosmic rays at earth: Researcher's reference, manual and data book, Amsterdam, Netherlands: Elsevier 2001.

[22] A. Haungs, H. Rebel, and M. Roth, Rept. Prog. Phys. 66 (2003) 1145-1206.

[23] V. Hess, Phys. Zeitschr. 13 (1912) 1084.

[24] T. Wulf, Phys. Zeitschr. 10 (1909) 997.

[25] W. Kolhörster, Phys. Zeitschr. 26 (1925) 654.

[26] W. Bothe and W. Kolhörster, Zeitschr. f. Phys. 56 (1929) 751.

[27] J. Clay, Amsterdam Proc. 33 (1930) 711.

[28] W. Kolhörster et al., Naturw. 26 (1938) 576.

[29] P. Auger et al., Comptes rendus 206 (1938) 1721.

[30] M. Schein et al., Phys. Rev. 59 (1941) 615.

[31] H. Brandt and B. Peters, Phys. Rev. 74 (1948) 1828.

[32] G. Kulikov and G. Khristiansen, JETP 35 (1958) 635.

[33] J. Linsley, Phys. Rev. Lett. 10 (1963) 146-148.

[34] C. J. Bell et al., J. Phys. A 12 (1974) 990.

[35] D. M. Edge, A. C. Evans, and H. J. Garmston, J. Phys. A 6 (1973) 1612.

[36] B. N. Afanasiev et al., in Proceedings of the Tokyo Workshop on Techniques for the Study of Extremely High Energy Cosmic Rays, edited by M. Nagano (Institute for Cosmic Ray Research, University of Tokyo, Tokyo, Japan), p.35, 2003.

[37] N. Chiba et al. (AGASA Collab.), Nucl. Instrum. Meth. A311 (1992) 338-349.

[38] R. M. Baltrusaitis et al. (Fly's Eye Collab.), Nucl. Instrum. Meth. A240 (1985) 410-428.

[39] J. Linsley, Proc of 8th Int. Cosmic Ray Conf., Jaipur 4 (1963) 77-99.

[40] D. J. Bird et al. (Fly's Eye Collab.), Phys. Rev. Lett. 71 (1993) 3401-3404.

[41] M. A. Lawrence, R. J. O. Reid, and A. A. Watson, J. Phys. G17 (1991) 733-757.

[42] M. Nagano et al., J. Phys. G18 (1992) 423-442. 
[43] C. Anderson, Phys. Rev. 43 (1933) 491.

[44] S. Neddermeyer and C. Anderson, Phys. Rev. 51 (1937) 884.

[45] C. Lattes et al., Nature 160 (1947) 453.

[46] K. Niu, E. Mikumo, and Y. Maeda, Prog. Theor. Phys. 46 (1971) 1644-1646.

[47] T. K. Gaisser and F. Halzen, Phys. Rev. D14 (1976) 3153.

[48] C. M. G. Lattes, Y. Fujimoto, and S. Hasegawa, Phys. Rept. 65 (1980) 151.

[49] S. A. Slavatinsky, Nucl. Phys. Proc. Suppl. 52B (1997) 56-70.

[50] J. Hörandel, Adv. Space Res. 38 (2006) 1549.

[51] J. Simpson, Ann. Rev. Nucl. Part. Sci. 33 (1983) 323-381.

[52] P. Fowler et al., Astrophys. J. 314 (1987) 739.

[53] P. Fowler et al., Nucl. Instrum. Meth. 147 (1977) 195.

[54] W. Binns et al., Astrophys. J. 346 (1989) 997.

[55] E. Shirk et al., Astrophys. J. 220 (1978) 719.

[56] D. Lawrence et al., Nucl. Instrum. Meth. A 420 (1999) 402.

[57] B. Weaver et al., Proc. of 27th Int. Cosmic Ray Conf., Hamburg 5 (2001) 1720.

[58] J. Tueller and M. Israel, from W.R. Binns et al., Ap. J. 247 (1981) L115.

[59] J. Donelly et al., Proc. of 26th Int. Cosmic Ray Conf., Salt Lake City 3 (1999) 109.

[60] K. Lodders, Astrophys. J. 591 (2003) 1220.

[61] S. Stephens and R. Streitmatter, Astrophys. J. 505 (1998) 266.

[62] A. Molnar and M. Simon, Proc. of 28th Int. Cosmic Ray Conf., Tsukuba 4 (2003) 1937.

[63] N. Yanasak et al., Astrophys. J. 563 (2001) 768.

[64] W. Baade and F. Zwicky, Phys. Rev. 46 (1934) 76.

[65] E. Fermi, Phys. Rev. 75 (1949) 1169.

[66] W. Axford et al., Proc. of 15th Int. Cosmic Ray Conf., Plovdiv 11 (1977) 132.

[67] G. Krymsky, Dok. Acad. Nauk USSR 234 (1977) 1306.

[68] A. Bell, Mon. Not. R. Astron. Soc. 182 (1978) 147.

[69] R. D. Blandford and J. P. Ostriker, Astrophys. J. 221 (1978) L29-L32.

[70] R. Blandford and D. Eichler, Phys. Rept. 154 (1987) 1-75.

[71] P. Lagage and C. Cesarsky, Astron. Astroph. 125 (1983) 249.

[72] E. Berezhko, Astropart. Phys. 5 (1996) 367.

[73] S. G. Lucek and A. R. Bell, Mon. Not. R. Astron. Soc. 314 (2000) 65-74.

[74] M. Wiedenbeck et al., Proc. of 28th Int. Cosmic Ray Conf., Tsukuba 4 (2003) 1899.

[75] B. Peters, Il Nuovo Cimento 22 (1961) 800.

[76] T. K. Gaisser, Neutrinos and Explosive Events in the Universe, page 3 M.M. Shapiro, T. Stanev, J.P. Wefel (eds.) NATO Science Series, Springer 2005.

[77] K. Greisen, Phys. Rev. Lett. 16 (1966) 748-750.

[78] G. T. Zatsepin and V. A. Kuzmin, Pis'ma Zh. Eksp. Teor. Fiz. 4 (1966) 114.

[79] C. T. Hill and D. N. Schramm, Phys. Rev. D31 (1985) 564.

[80] V. S. Berezinsky and S. I. Grigoreva, Astron. Astrophys. 199 (1988) 1. 
[81] S. Yoshida and M. Teshima, Prog. Theor. Phys. 89 (1993) 833-845.

[82] T. Stanev, R. Engel, A. Mücke, R. J. Protheroe, and J. P. Rachen, Phys. Rev. D62 (2000) 093005

[83] F. W. Stecker and M. H. Salamon, Astrophys. J. 512 (1999) 521-526

[84] T. Yamamoto, K. Mase, M. Takeda, N. Sakaki, and M. Teshima, Astropart. Phys. 20 (2004) 405-412

[85] E. Khan et al., Astropart. Phys. 23 (2005) 191-201

[86] D. Allard et al., JCAP 0609 (2006) 005

[87] Z. Fodor, S. D. Katz, and A. Ringwald, Proc. of Physics Beyond the Standard Model: Beyond the Desert 02 (2002) 263-267 and hep-ph/0210123.

[88] H. Blümer and K. H. Kampert, Phys. Bl. 56N3 (2000) 39-45.

[89] P. Blasi, R. I. Epstein, and A. V. Olinto, Astrophys. J. 533 (2000) L123

[90] R. J. Protheroe and A. P. Szabo, Phys. Rev. Lett. 69 (1992) 2885-2888.

[91] E. G. Berezhko, Astrophys. J. 684 (2008) L69-L71

[92] P. L. Biermann et al., to appear in Proc. of COsmic Ray Int. Seminar (CRIS 2008)", Malfa (Salina Island - Italy) on September 15-19, 2008 (2008) and arXiv:0811.1848 [astro-ph].

[93] J. P. Rachen and P. L. Biermann, Astron. Astrophys. 272 (1993) 161-175

[94] M. J. Hardcastle, C. C. Cheung, I. J. Feain, and L. Stawarz, arXiv:0808.1593 [astro-ph].

[95] E. Waxman, Phys. Rev. Lett. 75 (1995) 386-389

[96] M. Vietri, Astrophys. J. 453 (1995) 883-889

[97] K. Murase, K. Ioka, S. Nagataki, and T. Nakamura, Phys. Rev. D78 (2008) 023005

[98] V. Berezinsky, M. Kachelrieß, and A. Vilenkin, Phys. Rev. Lett. 79 (1997) 4302-4305

[99] R. Aloisio, V. Berezinsky, and M. Kachelrieß, Phys. Rev. D74 (2006) 023516

[100] J. R. Ellis, V. E. Mayes, and D. V. Nanopoulos, Phys. Rev. D74 (2006) 115003

[101] C. T. Hill, Nucl. Phys. B224 (1983) 469.

[102] R. J. Protheroe and P. A. Johnson, Astropart. Phys. 4 (1996) 253

[103] V. Berezinsky, P. Blasi, and A. Vilenkin, Phys. Rev. D58 (1998) 103515

[104] S. Sarkar and R. Toldra, Nucl. Phys. B621 (2002) 495-520

[105] S. R. Coleman and S. L. Glashow, Phys. Rev. D59 (1999) 116008

[106] J. R. Ellis, N. E. Mavromatos, and D. V. Nanopoulos, Phys. Rev. D63 (2001) 124025

[107] M. Jankiewicz, R. V. Buniy, T. W. Kephart, and T. J. Weiler, Astropart. Phys. 21 (2004) 651-666

[108] S. T. Scully and F. W. Stecker, Astropart. Phys. 31 (2008) 220-225

[109] W. Bietenholz, arXiv:0806.3713 [hep-ph], and references therein.

[110] T. J. Weiler, Astropart. Phys. 11 (1999) 303.

[111] D. Fargion, B. Mele, and A. Salis, Astrophys. J. 517 (1999) 725.

[112] M. Ahlers, A. Ringwald, and H. Tu, Astropart. Phys. 24 (2006) 438-450

[113] I. F. M. Albuquerque, G. R. Farrar, and E. W. Kolb, Phys. Rev. D59 (1999) 015021

[114] M. Kachelrieß, D. V. Semikoz, and M. A. Tortola, Phys. Rev. D68 (2003) 043005

[115] M. Kachelrieß, Comptes Rendus Physique 5 (2004) 441-452

[116] T. Stanev, Astrophys. J. 479 (1997) 290

[117] P. G. Tinyakov and I. I. Tkachev, Astropart. Phys. 18 (2002) 165-172 
[118] J. Alvarez-Muniz, R. Engel, and T. Stanev, Astrophys. J. 572 (2001) 185-201

[119] P. P. Kronberg, Rep. Prog. Phys. 57 (1994) 325.

[120] G. Sigl, F. Miniati, and T. A. Ensslin, Phys. Rev. D70 (2004) 043007

[121] K. Dolag, D. Grasso, V. Springel, and I. Tkachev, JCAP 0501 (2005) 009

[122] D. V. Semikoz and G. Sigl, JCAP 0404 (2004) 003

[123] D. Seckel and T. Stanev, Phys. Rev. Lett. 95 (2005) 141101

[124] G. Gelmini, O. Kalashev, and D. V. Semikoz, J. Exp. Theor. Phys. 106 (2008) 1061-1082

[125] D. Allard, E. Parizot, and A. V. Olinto, Astropart. Phys. 27 (2007) 61-75

[126] J. K. Becker, Phys. Rept. 458 (2008) 173-246

[127] T. K. Gaisser, Cosmic rays and particle physics, Cambridge Univ. Press, Cambridge, 1990.

[128] T. Stanev, High energy cosmic rays, Springer Berlin 2003.

[129] M. Lemoine and G. Sigl (eds.), Physics and astrophysics of ultra high energy cosmic rays, Springer Verlag Berlin, Heidelberg 2001.

[130] W. Heitler, Quantum Theory of Radiation, Oxford University Press Oxford 1944 2nd edition.

[131] B. Rossi and K. Greisen, Rev. Mod. Phys. 13 (1941) 240.

[132] K. Kamata and J. Nishimura, Prog. Theo. Phys. 6 (1958) 93.

[133] J. Nishimura, Handbuch der Physik 46/2 (1965) 1.

[134] K. Greisen, Prog. Cosmic Ray Physics 3 (1965) 1.

[135] P. Lipari, Phys. Rev. D 79 (2009) 063001.

[136] T. K. Gaisser and A. M. Hillas, in Proc. 15th Int. Cosmic Ray Conf., p. 358, Plovdiv, Bulgaria, 1977.

[137] G. Z. Molière, Z. Naturforsch 3a (1948) 78.

[138] K. Greisen, Ann. Rev. Nucl. Sci. 10 (1960) 63.

[139] L. D. Landau and I. Pomeranchuk, Dokl. Akad. Nauk Ser. Fiz. 92 (1953) 535-536.

[140] A. B. Migdal, Phys. Rev. 103 (1956) 1811-1820.

[141] T. Stanev, C. Vankov, R. E. Streitmatter, R. W. Ellsworth, and T. Bowen, Phys. Rev. D25 (1982) 1291-1304.

[142] S. Klein, Rev. Mod. Phys. 71 (1999) 1501-1538

[143] T. Erber, Rev. Mod. Phys. 38 (1966) 626-659.

[144] T. Stanev and H. P. Vankov, Phys. Rev. D55 (1997) 1365-1371

[145] X. Bertou, P. Billoir, and S. Dagoret-Campagne, Astropart. Phys. 14 (2000) 121.

[146] H. P. Vankov, N. Inoue, and K. Shinozaki, Phys. Rev. D67 (2003) 043002

[147] P. Homola et al., Comput. Phys. Commun. 173 (2005) 71

[148] P. Homola et al., Astropart. Phys. 27 (2007) 174-184

[149] M. Risse and P. Homola, Mod. Phys. Lett. A22 (2007) 749-766

[150] J. Matthews, Astropart. Phys. 22 (2005) 387-397.

[151] M. Risse, Acta Phys. Polon. B35 (2004) 1787-1797

[152] T. Pierog, R. Engel, and D. Heck, Czech. J. Phys. 56 (2006) A161-A172

[153] J. Hörandel, Mod. Phys. Lett. A 22 (2007) 1533. 
[154] J. Alvarez-Muniz, R. Engel, T. K. Gaisser, J. A. Ortiz, and T. Stanev, Phys. Rev. D66 (2002) 033011

[155] C. Meurer, J. Blümer, R. Engel, A. Haungs, and M. Roth, Czech. J. Phys. 56 (2006) A211

[156] J. Linsley and A. A. Watson, Phys. Rev. Lett. 46 (1981) 459-463.

[157] J. Engel, T. K. Gaisser, T. Stanev, and P. Lipari, Phys. Rev. D46 (1992) 5013-5025.

[158] J. Knapp, D. Heck, and G. Schatz, in Wissenschaftliche Berichte FZKA 5828, Forschungszentrum Karlsruhe, 1996.

[159] J. Knapp, D. Heck, S. J. Sciutto, M. T. Dova, and M. Risse, Astropart. Phys. 19 (2003) 77-99

[160] J. Ranft, Phys. Rev. D51 (1995) 64-84.

[161] S. Roesler, R. Engel, and J. Ranft, Proc of 27th Int. Cosmic Ray Conf., Hamburg (2) 439.

[162] K. Werner, F.-M. Liu, and T. Pierog, Phys. Rev. C74 (2006) 044902

[163] T. Pierog and K. Werner, Phys. Rev. Lett. 101 (2008) 171101

[164] N. N. Kalmykov and S. Ostapchenko, Sov. J. Nucl. Phys. 50 (1989) (2) 315.

[165] N. N. Kalmykov and S. S. Ostapchenko, Phys. Atom. Nucl. 56 (1993) 346-353.

[166] N. N. Kalmykov, S. S. Ostapchenko, and A. I. Pavlov, Nucl. Phys. Proc. Suppl. 52B (1997) 17-28.

[167] S. Ostapchenko, Phys. Rev. D74 (2006) 014026

[168] S. Ostapchenko, Phys. Lett. B636 (2006) 40-45

[169] R. S. Fletcher, T. K. Gaisser, P. Lipari, and T. Stanev, Phys. Rev. D50 (1994) 5710-5731.

[170] R. Engel, T. K. Gaisser, T. Stanev, and P. Lipari, Proc. of 26th Int. Cosmic Ray Conf., Salt Lake City 1 (1999) 415.

[171] R. Engel and H. Rebel, Acta Phys. Polon. B35 (2004) 321-330.

[172] D. Heck, Talk given at CORSIKA School 2008, Freudenstadt, Germany, 25 - 30 November 2008 , available at http://www-ik.fzk.de/corsika/corsika-school2008/, 2008.

[173] F. Ballarini et al., J. Phys. Conf. Ser. 41 (2006) 151-160.

[174] H. Fesefeldt, preprint PITHA-85/02, RWTH Aachen, 1985.

[175] M. Bleicher et al., J. Phys. G: Nucl. Part. Phys. 25 (1999) 1859.

[176] R. Engel, T. K. Gaisser, P. Lipari, and T. Stanev, Proc. of 27th Int. Cosmic Ray Conf., Hamburg (2001) 1381.

[177] A. M. Hillas, Nucl. Phys. Proc. Suppl. 52B (1997) 29-42.

[178] R. Engel, T. K. Gaisser, and T. Stanev, Proc of 29th Int. Symposium on Multiparticle Dynamics (ISMD 99), Providence, Rhode Island, 8-13 Aug 1999, p. 457.

[179] H.-J. Drescher and G. R. Farrar, Astropart. Phys. 19 (2003) 235-244

[180] D. Heck et al., 28th Int. Cosmic Ray Conf., Tsukuba 1 (2003) 279.

[181] H.-J. Drescher, M. Bleicher, S. Soff, and H. Stoecker, Astropart. Phys. 21 (2004) 87-94

[182] D. Heck, J. Knapp, J. Capdevielle, G. Schatz, and T. Thouw, Wissenschaftliche Berichte FZKA 6019, Forschungszentrum Karlsruhe, 1998.

[183] T. Bergmann et al., Astropart. Phys. 26 (2007) 420-432

[184] T. Pierog et al., Proc. 29th Int. Cosmic Ray Conf., Pune 7 (2005) 103.

[185] D. Heck, private communication, 2006.

[186] C. Pajares, D. Sousa, and R. A. Vazquez, Phys. Rev. Lett. 86 (2001) 1674-1677 
[187] J. Dias de Deus, M. C. Espirito Santo, M. Pimenta, and C. Pajares, Phys. Rev. Lett. 96 (2006) 162001

[188] J. Alvarez-Muniz et al., Astropart. Phys. 27 (2007) 271-277

[189] A. M. Hillas, J. Phys. G8 (1982) 1461-1473.

[190] M. Giller, A. Kacperczyk, J. Malinowski, W. Tkaczyk, and G. Wieczorek, J. Phys. G31 (2005) 947-958.

[191] F. Nerling, J. Blümer, R. Engel, and M. Risse, Astropart. Phys. 24 (2006) 421-437

[192] A. S. Chou et al., Proc. of 29th Int. Cosmic Ray Conf., Pune (2005) 319.

[193] F. Schmidt, M. Ave, L. Cazon, and A. S. Chou, Astropart. Phys. 29 (2008) 355-365

[194] M. Giller, H. Stojek, and G. Wieczorek, Int. J. Mod. Phys. A20 (2005) 6821-6824.

[195] S. Lafebre, R. Engel, H. Falcke, J. Hörandel, T. Huege, J. Kuijpers, and R. Ulrich, Astropart. Phys. 31 (2009) 243-254

[196] M. Aglietta et al. (EAS-TOP Collab.), Nucl. Instrum. Meth. A 336 (1993) 310.

[197] T. Antoni et al. (KASCADE Collab.), Nucl. Instrum. Meth. A 513 (2003) 490.

[198] G. Navarra et al. (KASCADE-Grande Collab.), Nucl. Instrum. Meth. A 518 (2004) 207.

[199] J. Abraham et al. (Pierre Auger Collab.), Nucl. Instrum. Meth. A523 (2004) 50-95.

[200] J. Linsley, L. Scarsi, and B. Rossi, Phys. Rev. Lett. 6 (1961) 485-487.

[201] K. Bernlöhr et al., Astropart. Phys. 8 (1998) 253.

[202] P. Doll et al., Nucl. Instrum. Meth. A 488 (2002) 517.

[203] M. Calicchio et al., Nucl. Instrum. Meth. A 264 (1988) 18.

[204] M. Ambrosio et al., Nucl. Instrum. Meth. A 486 (2002) 663.

[205] R. A. Falcone et al. (EAS-TOP Collab.), Nucl. Instrum. Meth. A 420 (1999) 117.

[206] J. Engler et al., Nucl. Instrum. Meth. A 427 (1999) 528.

[207] C. Bonifazi et al. (Pierre Auger Collab.), to appear in Proc. of Cosmic Ray Int. Seminar (CRIS 2008), Malfa (2009) and arXiv:0901.3138 [astro-ph.HE].

[208] T. Antoni et al. (KASCADE Collab.), Astropart. Phys. 14 (2001) 245.

[209] T. Antoni et al. (KASCADE Collab.), Astropart. Phys. 24 (2006) 467.

[210] S. Yoshida et al. (AGASA Collab.), J. Phys. G: Nucl. Part. Phys. 20 (1994) 651.

[211] J. Linsley et al., J. Phys. Soc. Japan 17 (1962) Suppl. A-III 91.

[212] R. Coy et al., Astropart. Phys. 6 (1997) 263.

[213] D. Barnhill et al. (Pierre Auger Collab.), Proc. of 29th Int. Cosmic Ray Conf., Pune 7 (2005) 291 and astro-ph/0507590

[214] T. Antoni et al. (KASCADE Collab.), J. Phys. G: Nucl. Part. Phys. 25 (1999) 2161.

[215] M. Glasmacher et al., Astropart. Phys. 10 (1999) 291.

[216] R. Glasstetter et al. (KASCADE Collab.), Proc. of 29th Int. Cosmic Ray Conf., Pune 6 (2005) 293.

[217] M. Takeda et al. (AGASA Collab.), Astropart. Phys. 19 (2003) 447-462

[218] A. Hillas et al., Proc. of 12th Int. Cosmic Ray Conf., Hobart 3 (1971) 1001.

[219] H. Y. Dai, K. Kasahara, Y. Matsubara, M. Nagano, and M. Teshima, J. Phys. G14 (1988) 793-805.

[220] N. Sakaki et al. (AGASA Collab.), Proc. of 27th Int. Cosmic Ray Conf., Hamburg 1 (2001) 333.

[221] J. Abraham et al. (Pierre Auger Collab.), Phys. Rev. Lett. 101 (2008) 061101 
[222] M. Ave et al., Astropart. Phys. 19 (2003) 61-75

[223] M. Ave, J. Knapp, M. Marchesini, M. Roth, and A. A. Watson, Proc. of 28th Int. Cosmic Ray Conf., Tsukuba (2003) 349.

[224] M. Giller, G. Wieczorek, A. Kacperczyk, H. Stojek, and W. Tkaczyk, J. Phys. G30 (2004) 97-105.

[225] J. Fowler et al. (BLANCA Collab.), Astropart. Phys. 15 (2001) 49.

[226] F. Arqueros et al., Astron. Astroph. 359 (2000) 682.

[227] O. Gress et al., Proc. of 25th Int. Cosmic Ray Conf., Durban 4 (1997) 129.

[228] O. Gress et al., Nucl. Phys. B (Proc. Suppl.) 75A (1999) 299.

[229] D. Chernov et al., Int. J. Mod. Phys. A 20 (2005) 6799.

[230] D. Chernov et al., Int. J. Mod. Phys. A 20 (2005) 6796.

[231] G. Mohanty et al., Astropart. Phys. 9 (1998) 15.

[232] J. Hinton, New Astronomy Reviews 48 (2004) 331.

[233] D. Ferenc et al., Nucl. Instrum. Meth. A 553 (2005) 274.

[234] T. Weekes et al., Astropart. Phys. 17 (2002) 221.

[235] S. Swordy and D. Kieda, Astropart. Phys. 13 (2000) 137.

[236] F. Arciprete et al., Nucl. Phys. Proc. Suppl. 150 (2006) 186-189.

[237] M. Ave et al. (AIRFLY Collab.), Nucl. Instrum. Meth. A 597 (2008) 41.

[238] B. Keilhauer, J. Blümer, R. Engel, and H. O. Klages, Astropart. Phys. 25 (2006) 259-268

[239] F. Kakimoto et al., Nucl. Instrum. Meth. A372 (1996) 527-533.

[240] M. Nagano, K. Kobayakawa, N. Sakaki, and K. Ando, Astropart. Phys. 20 (2003) 293-309

[241] J. W. Belz et al. (FLASH Collab.), Astropart. Phys. 25 (2006) 129-139

[242] P. Colin et al. (MACFLY Collab.), Astropart. Phys. 27 (2007) 317-325

[243] T. Waldenmaier, J. Blümer, and H. Klages, Astropart. Phys. 29 (2008) 205-222

[244] M. Ave et al. (AIRFLY Collab.), Astropart. Phys. 28 (2007) 41

[245] R. Abbasi et al., Astropart. Phys. 29 (2008) 77-86

[246] F. Arqueros, J. Hörandel, and B. Keilhauer (eds.), Nucl. Instrum. Meth. A 597 (2008) 1-120.

[247] F. Arqueros, F. Blanco, and J. Rosado, Nucl. Instrum. Meth. A597 (2008) 94-98

[248] M. Risse and D. Heck, Astropart. Phys. 20 (2004) 661

[249] H. M. J. Barbosa, F. Catalani, J. A. Chinellato, and C. Dobrigkeit, Astropart. Phys. 22 (2004) $159-166$

[250] D. Kuempel, K. H. Kampert, and M. Risse, Astropart. Phys. 30 (2008) 167-174

[251] R. U. Abbasi et al. (HiRes Collab.), Astropart. Phys. 27 (2007) 512-520

[252] B. Fick et al. (Pierre Auger Collab.), 28th Int. Cosmic Ray Conf., Tsukuba (2003) 449

[253] M. Unger, B. R. Dawson, R. Engel, F. Schüssler, and R. Ulrich, Nucl. Instrum. Meth. A588 (2008) 433-441

[254] M. Giller and G. Wieczorek, Astropart. Phys. 31 (2009) 212-219.

[255] R. Abbasi et al. (HiRes Collab.), Astropart. Phys. 25 (2006) 74-83

[256] S. Y. BenZvi et al., Nucl. Instrum. Meth. A574 (2007) 171-184

[257] B. Keilhauer, J. Blümer, R. Engel, H. O. Klages, and M. Risse, Astropart. Phys. 22 (2004) 249-261

[258] D. J. Bird et al. (Fly's Eye Collab.), Astrophys. J. 441 (1995) 144-150. 
[259] H. Kawai et al. (TA Collab.), Nucl. Phys. Proc. Suppl. 175-176 (2008) 221-226.

[260] http://www-ta.icrr.u-tokyo.ac.jp/TA_Proposal/

[261] G. Askaryan, JETP 48 (1965) 988.

[262] J. Jelley et al., Nature 205 (1965) 327.

[263] H. Allan, Progress in Elementary Particles and Cosmic Ray Physics, page 169 J.G. Wilson S.G. Wouthuysen eds., North Holland 1971.

[264] D. Ardouin et al. (CODALEMA Collab.), Nucl. Instrum. Meth. A555 (2005) 148-163.

[265] A. Horneffer et al. (LOPES Collab.), Proc. of the SPIE 5500 (2004) 129.

[266] H. Falcke et al. (LOPES Collab.), Nature 435 (2005) 313-316

[267] D. Ardouin et al. (CODALEMA Collab.), Astropart. Phys. 26 (2006) 341-350

[268] A. M. van den Berg (Pierre Auger Collab.), Proc. of 30th Int. Cosmic Ray Conf., Merida 5 (2007) 885 and arXiv:0708.1709 [astro-ph].

[269] H. Falcke et al. (LOPES Collab.), arXiv:0804.0548 [astro-ph].

[270] E. Zas, F. Halzen, and T. Stanev, Phys. Rev. D45 (1992) 362-376.

[271] D. Saltzberg et al., Phys. Rev. Lett. 86 (2001) 2802.

[272] P. W. Gorham et al. (ANITA Collab.), Phys. Rev. Lett. 99 (2007) 171101

[273] T. Huege and H. Falcke, Astron. Astrophys. 412 (2003) 19-34

[274] T. Huege, R. Ulrich, and R. Engel, Astropart. Phys. 27 (2007) 392-405

[275] T. Huege, R. Ulrich, and R. Engel, Astropart. Phys. 30 (2008) 96-104

[276] R. Engel, N. N. Kalmykov, and A. A. Konstantinov, Int. J. Mod. Phys. A21S1 (2006) 65-69.

[277] O. Scholten, K. Werner, and F. Rusydi, Astropart. Phys. 29 (2008) 94-103

[278] K. Werner and O. Scholten, Astropart. Phys. 29 (2008) 393-411

[279] T. Gousset, J. Lamblin, and S. Valcares, Astropart. Phys. 31 (2009) 52-62.

[280] H. S. Ahn et al. (ATIC-2 Collab.), Proc. of 28th Int. Cosmic Ray Conf., Tsukuba (2003) 1853.

[281] J. Wefel, private communication, 2006.

[282] N. L. Grigorov et al., Yad. Fiz. 11 (1970) 1058.

[283] N. L. Grigorov et al., Proc. of 12th Int. Cosmic Ray Conf., Hobart 2 (1971) 206.

[284] V. Derbina et al., Astrophys. J. 628 (2005) L41.

[285] M. Amenomori et al. (Tibet AS $\gamma$ Collab.), Astrophys. J. 678 (2008) 1165-1179

[286] T. Antoni et al. (KASCADE Collab.), Astropart. Phys. 24 (2005) 1-25

[287] J. C. Arteaga-Velazques et al. (KASCADE-Grande Collab.), to appear in Proc. of 15th Int. Symposium on Very High-Energy Cosmic Ray Interactions, Paris, 2008.

[288] M. Nagano et al., J. Phys. G10 (1984) 1295.

[289] T. Abu-Zayyad et al. (HiRes-MIA Collab.), Astrophys. J. 557 (2001) 686-699

[290] T. Abu-Zayyad et al. (HiRes-MIA Collab.), Phys. Rev. Lett. 84 (2000) 4276

[291] R. Abbasi et al. (HiRes Collab.), Phys. Rev. Lett. 100 (2008) 101101

[292] K. Asakimori et al., Proc. of 24th Int. Cosmic Ray Conf., Rome 2 (1995) 707.

[293] I. Ivanenko et al., Proc. of 23rd Int. Cosmic Ray Conf., Calgary 2 (1993) 17.

[294] S. Ogio et al., Astrophys. J. 612 (2004) 268.

[295] M. Aglietta et al. (EAS-TOP Collab.), Astropart. Phys. 10 (1999) 1. 
[296] J. Hörandel et al. (KASCADE Collab.), Proc. of 26th Int. Cosmic Ray Conf., Salt Lake City 1 (1999) 337.

[297] T. Antoni et al. (KASCADE Collab.), Astropart. Phys. 16 (2002) 245.

[298] Y. Fomin et al., Proc. of 22nd Int. Cosmic Ray Conf., Dublin 2 (1991) 85.

[299] N. Ito et al., Proc. of 25th Int. Cosmic Ray Conf., Durban 4 (1997) 117.

[300] M. Amenomori et al. (Tibet AS $\gamma$ Collab.), Phys. Rev. D 62 (2000) 072007.

[301] J. Alcaraz et al., Phys. Lett. B 490 (2000) 27.

[302] J. Wefel et al., Proc. of 29th Int. Cosmic Ray Conf., Pune 3 (2005) 105.

[303] T. Sanuki et al., Astrophys. J. 545 (2000) 1135.

[304] M. Boezio et al., Astropart. Phys. 19 (2003) 583.

[305] M. D. Vernois et al., Proc. of 27th Int. Cosmic Ray Conf., Hamburg 5 (2001) 1618.

[306] M. Ichimura et al., Phys. Rev. D 48 (1993) 1949.

[307] M. Menn et al., Astrophys. J. 533 (2000) 281.

[308] K. Asakimori et al., Astrophys. J. 502 (1998) 278.

[309] R. Bellotti et al., Phys. Rev. D 60 (1999) 052002.

[310] P. Papini et al., Proc. of 23rd Int. Cosmic Ray Conf., Calgary 1 (1993) 579.

[311] E. Diehl et al., Astropart. Phys. 18 (2003) 487.

[312] M. Ryan et al., Phys. Rev. Lett. 28 (1972) 985.

[313] L. Smith et al., Astrophys. J. 180 (1973) 987.

[314] V. Zatsepin et al., Proc. of 23rd Int. Cosmic Ray Conf., Calgary 2 (1993) 14.

[315] T. Antoni et al. (KASCADE Collab.), Astrophys. J. 612 (2004) 914.

[316] G. Navarra et al. (EAS-TOP Collab.), Proc. of 28th Int. Cosmic Ray Conf., Tsukuba 1 (2003) 147.

[317] M. Aglietta et al. (EAS-TOP Collab.), Astropart. Phys. 19 (2003) 329.

[318] Y. Hayashi et al., Proc. of 29th Int. Cosmic Ray Conf., Pune 10 (2005) 243.

[319] F. Aharonian et al., Phys. Rev. D 59 (1999) 092003.

[320] N. Inoue et al., Proc. of 25th Int. Cosmic Ray Conf., Durban 4 (1997) 113.

[321] J. Huang et al., Astropart. Phys. 18 (2003) 637.

[322] M. Amenomori et al., Phys. Rev. D 62 (2000) 112002.

[323] M. Amenomori et al. (Tibet AS $\gamma$ Collab.), Proc. of 28th Int. Cosmic Ray Conf., Tsukuba 1 (2003) 107.

[324] W. Webber et al., Proc. of 20th Int. Cosmic Ray Conf., Moscow 1 (1987) 325.

[325] M. Cherry, J. Phys.: Conf. Ser. 47 (2006) 31.

[326] D. Müller et al., Astrophys. J. 374 (1991) 356.

[327] D. Müller et al., Proc. of 29th Int. Cosmic Ray Conf., Pune 3 (2005) 89.

[328] JACEE collaboration after T. Shibata, Nucl. Phys. B (Proc. Suppl.) 75A (1999) 22.

[329] J. Engelmann et al., Astron. Astroph. 148 (1985) 12.

[330] E. Juliusson et al., Astrophys. J. 191 (1974) 331.

[331] G. Minagawa et al., Astrophys. J. 248 (1981) 847.

[332] M. Hareyama et al., Proc. of 26th Int. Cosmic Ray Conf., Salt Lake City 3 (1999) 105. 
[333] F. Aharonian et al. (H.E.S.S. Collab.), Phys. Rev. D75 (2007) 042004

[334] M. Amenomori et al., Proc. of 28th Int. Cosmic Ray Conf., Tsukuba 1 (2003) 143.

[335] D. Kieda et al., Astropart. Phys. 15 (2001) 287.

[336] J. Hörandel, Adv. Space Res. 41 (2008) 442.

[337] R. U. Abbasi et al. (HiRes Collab.), Astropart. Phys. 30 (2008) 175-179

[338] M. I. Pravdin, private communication, 2004.

[339] L. Anchordoqui and H. Goldberg, Phys. Lett. B583 (2004) 213-221

[340] D. J. Bird et al. (Fly's Eye Collab.), Astrophys. J. 424 (1994) 491-502.

[341] M. Ave, J. Knapp, J. Lloyd-Evans, M. Marchesini, and A. A. Watson, Astropart. Phys. 19 (2003) $47-60$

[342] M. Takeda et al. (AGASA Collab.), Proc. of 28th Int. Cosmic Ray Conf., Tsukuba (2003) 381.

[343] Y. A. Fomin et al. (Yakutsk Collab.), Proc. of 22th Int. Cosmic Ray Conf., Dublin 2 (1991) 85.

[344] A. V. Glushkov et al., Proc. of 28th Int. Cosmic Ray Conf., Tsukuba (2003) 389.

[345] R. Aloisio et al., Astropart. Phys. 27 (2007) 76-91

[346] V. Berezinsky, J. Phys. Conf. Ser. 120 (2008) 012001

[347] J. N. Bahcall and E. Waxman, Phys. Lett. B556 (2003) 1-6

[348] T. Yamamoto (Pierre Auger Collab.), Proc. of 30th Int. Cosmic Ray Conf., Merida 4 (2007) 335

[349] R. U. Abbasi et al. (HiRes Collab.), Phys. Lett. B619 (2005) 271-280

[350] V. Berezinsky, A. Gazizov, and S. Grigorieva, astro-ph/0302483.

[351] V. Berezinsky, A. Z. Gazizov, and S. I. Grigorieva, Phys. Rev. D74 (2006) 043005

[352] D. De Marco, P. Blasi, and A. V. Olinto, Astropart. Phys. 20 (2003) 53-65

[353] M. Teshima, talk given at the XXXIII International Symposium on Multiparticle Dynamics (ISMD), Cracow, Poland, Sep 5-11, 2003, 2004.

[354] A. S. Chou, Phys. Rev. D74 (2006) 103001

[355] M. Nagano, D. Heck, K. Shinozaki, N. Inoue, and J. Knapp, Astropart. Phys. 13 (2000) 277-294

[356] H.-J. Drescher and G. R. Farrar, Astropart. Phys. 24 (2005) 372-381

[357] R. Engel (Pierre Auger Collab.), Proc. of 30th Int. Cosmic Ray Conf., Merida 4 (2007) 385 and arXiv:0706.1921 [astro-ph].

[358] F. Schmidt et al. (Pierre Auger Collab.), to appear in Proc. of XV Int. Symposium on Very High Energy Cosmic Ray Interactions, Paris (2009) and arXiv:0902.4613 [astro-ph.HE].

[359] K. Shinozaki, private communication, 2006.

[360] C. Song et al. (HiRes Collab.), Astropart. Phys. 14 (2000) 7-13

[361] T. Abu-Zayyad et al. (HiRes Collab.), Astropart. Phys. 23 (2005) 157-174

[362] R. U. Abbasi et al. (HiRes Collab.), Phys. Rev. Lett. 92 (2004) 151101

[363] R. Abbasi et al. (HiRes Collab.), Astropart. Phys. 27 (2007) 370-381

[364] B. R. Dawson et al. (Pierre Auger Collab.), Proc. of 30th Int. Cosmic Ray Conf., Merida 4 (2007) 425 and arXiv:0706.1105 [astro-ph].

[365] M. Unger (Pierre Auger Collab.), Proc of 30th Int. Cosmic Ray Conf., Merida 4 (2007) 373 and arXiv:0706.1495 [astro-ph].

[366] S. Paling et al., Proc. of 25th Int. Cosmic Ray Conf., Durban 5 (1997) 253. 
[367] D. Bird et al. (Fly's Eye Collab.), Astrophys. J. 424 (1994) 491.

[368] A. Watson, Phys. Rep. 333 - 334 (2000) 309.

[369] R. U. Abbasi et al. (HiRes Collab.), Astrophys. J. 622 (2005) 910-926

[370] M. Cha et al., Proc. of 27th Int. Cosmic Ray Conf., Hamburg 1 (2001) 132.

[371] J. Dickinson et al., Proc. of 26th Int. Cosmic Ray Conf., Salt Lake City 3 (1999) 136.

[372] S. Knurenko et al., Proc. of 27th Int. Cosmic Ray Conf., Hamburg 1 (2001) 177.

[373] T. Pierog and K. Werner, Proc. of 30th Int. Cosmic Ray Conf., Merida 4 (2007) 629.

[374] M. Glasmacher et al., Astropart. Phys. 12 (1999) 1.

[375] C. Aguirre et al., Phys. Rev. D 62 (2000) 032003.

[376] M. Aglietta et al. (EAS-TOP Collab.), Astropart. Phys. 21 (2004) 583.

[377] M. Aglietta et al., Astropart. Phys. 20 (2004) 641.

[378] J. Hörandel et al. (KASCADE Collab.), Proc. 16th European Cosmic Ray Symposium, Alcala de Henares (1998) 579.

[379] K. Rawlins et al., Proc. of 28th Int. Cosmic Ray Conf., Tsukuba 1 (2003) 173.

[380] J. R. Hörandel, J. Phys. G29 (2003) 2439-2464

[381] J. Hörandel, Nucl. Phys. B (Proc. Suppl.) 151 (2006) 75.

[382] A. Apanasenko et al., Astropart. Phys. 16 (2001) 13.

[383] M. Aglietta et al., Astropart. Phys. 21 (2004) 223.

[384] T. K. Gaisser et al., Phys. Rev. D47 (1993) 1919-1932.

[385] L. K. Ding et al., Astrophys. J. 474 (1997) 490-495.

[386] D. Heck, M. Risse, and J. Knapp, Nucl. Phys. Proc. Suppl. 122 (2003) 364-367

[387] N. Hayashida et al. (AGASA Collab.), J. Phys. G21 (1995) 1101-1120.

[388] M. T. Dova, M. E. Mancenido, A. G. Mariazzi, T. P. McCauley, and A. A. Watson, Nucl. Phys. Proc. Suppl. 122 (2003) 235-238

[389] M. T. Dova, M. E. Mancenido, A. G. Mariazzi, T. P. McCauley, and A. A. Watson, Astropart. Phys. 21 (2004) 597-607

[390] K. Shinozaki (AGASA Collab.), Nucl. Phys. Proc. Suppl. 151 (2006) 3-10.

[391] R. Abbasi et al. (HiRes Collab.), Astropart. Phys. 26 (2006) 28-40

[392] M. N. Dyakonov et al., in Proceedings of the 23th Int. Cosmic Ray Conf., Calgary, Canada, vol. 4, p. 303, 1993.

[393] A. Borione et al., Nucl. Instrum. Meth. A346 (1994) 329-352.

[394] B. R. Dawson, R. Meyhandan, and K. M. Simpson, Astropart. Phys. 9 (1998) 331-338

[395] K. Shinozaki et al. (AGASA Collab.), Proc. of 28th Int. Cosmic Ray Conf., Tsukuba , 401.

[396] S. J. Sciutto, astro-ph/9911331.

[397] M. Ave, J. A. Hinton, R. A. Vazquez, A. A. Watson, and E. Zas, Phys. Rev. D65 (2002) 063007

[398] M. T. Dova, M. E. Mancenido, A. G. Mariazzi, T. P. McCauley, and A. A. Watson, Astropart. Phys. 21 (2004) 597-607.

[399] M. Chantell et al., Phys. Rev. Lett. 79 (1997) 1805.

[400] M. Aglietta et al. (EAS-TOP Collab.), Astropart. Phys. 6 (1996) 7191. 
[401] G. Schatz et al. (KASCADE Collab.), Proc. of 28th Int. Cosmic Ray Conf., Tsukuba 4 (2003) 2293.

[402] F. A. Aharonian and A. M. Atoyan, Astron. Astroph. 362 (2000) 937-952

[403] J. Abraham et al. (Pierre Auger Collab.), Astropart. Phys. 29 (2008) 243-256

[404] J. Abraham et al. (Pierre Auger Collab.), Astropart. Phys. 27 (2007) 155-168

[405] M. Ave et al., Phys. Rev. D 65 (2002) 063007.

[406] K. Shinozaki et al. (AGASA Collab.), Astrophys. J. 571 (2002) L117-L120.

[407] M. Risse et al., Phys. Rev. Lett. 95 (2005) 171102

[408] G. I. Rubtsov et al., Phys. Rev. D73 (2006) 063009

[409] A. Glushkov et al., JETP Lett. 85 (2007) 163.

[410] T. C. Weekes, arXiv:0811.1197 [astro-ph].

[411] J. Hinton, arXiv:0803.1609 [astro-ph].

[412] F. Halzen, R. A. Vazquez, T. Stanev, and H. P. Vankov, Astropart. Phys. 3 (1995) 151-156.

[413] M. Risse et al., Astropart. Phys. 21 (2004) 479-490

[414] M. Ave, J. A. Hinton, R. A. Vazquez, A. A. Watson, and E. Zas, Phys. Rev. Lett. 85 (2000) $2244-2247$

[415] R. Aloisio, V. Berezinsky, and M. Kachelrieß, Phys. Rev. D69 (2004) 094023

[416] J. Abraham et al. (Pierre Auger Collab.), Phys. Rev. Lett. 100 (2008) 211101

[417] O. Kalashev et al., Phys. Rev. D 66 (2002) 063004.

[418] F. Halzen and D. Hooper, Rept. Prog. Phys. 65 (2002) 1025-1078

[419] H. Falcke et al., New Astronomy Reviews 48 (2004) 1487.

[420] Y. Fukuda et al., Phys. Lett. B 436 (1998) 33.

[421] Y. Fukuda et al., Phys. Rev. Lett. 82 (1999) 1810.

[422] S. Ahmed et al., Phys. Rev. Lett. 92 (2004) 181301.

[423] K. Hirata et al., Phys. Rev. D 58 (1987) 1490.

[424] R. Bionta et al., Phys. Rev. Lett. 54 (1987) 1494.

[425] X. Bertou, P. Billoir, O. Deligny, C. Lachaud, and A. Letessier-Selvon, Astropart. Phys. 17 (2002) $183-193$

[426] J. L. Feng, P. Fisher, F. Wilczek, and T. M. Yu, Phys. Rev. Lett. 88 (2002) 161102

[427] E. Zas, New J. Phys. 7 (2005) 130

[428] C. Aramo et al., Astropart. Phys. 23 (2005) 65-77

[429] D. Fargion, Prog. Part. Nucl. Phys. 57 (2006) 384-393

[430] G. Miele, S. Pastor, and O. Pisanti, Phys. Lett. B634 (2006) 137-142

[431] D. Gora, M. Roth, and A. Tamburro, Astropart. Phys. 26 (2007) 402-413.

[432] A. Chilingarian et al., Astrophys. J. 597 (2003) L129.

[433] A. Chilingarian, G. Gharagyozian, G. Hovsepian, and G. Karapetian, Astropart. Phys. 25 (2006) 269-276.

[434] A. Erlykin and A. Wolfendale, Astropart. Phys. 22 (2004) 47.

[435] T. Antoni et al. (KASCADE Collab.), Astrophys. J. 608 (2004) 865.

[436] M. Amenomori et al., Astrophys. J. 635 (2005) L53. 
[437] M. Amenomori et al., Astrophys. J. 633 (2005) 1005.

[438] Y. Oyama et al., preprint astro-ph/0605020 2006.

[439] G. Guillian et al., Phys. Rev. D 75 (2007) 062003.

[440] M. Amenomori et al., Science 314 (2006) 439.

[441] A. Compton and I. Getting, Phys. Rev. 47 (1935) 817.

[442] T. Antoni et al. (KASCADE Collab.), Astrophys. J. 604 (2004) 687.

[443] J. Goodman, Neutrinos and Explosive Events in the Universe, page 243 M.M. Shapiro, T. Stanev, J.P. Wefel (eds.) NATO Science Series, Springer 2005.

[444] A. Abdo et al., Astrophys. J. 658 (2007) L33.

[445] A. Abdo et al., arXiv:0801.3827 2008.

[446] M. Salvati and B. Sacco, arXiv:0802.2181 2008.

[447] L. Drury and F. Aharonian, Astropart. Phys. 29 (2008) 420.

[448] K. Nagashima et al., Proc. of 21st Int. Cosmic Ray Conf., Adelaide 3 (1990) 180.

[449] M. Aglietta et al. (EAS-TOP Collab.), Astrophys. J. 470 (1996) 501.

[450] M. Aglietta et al., Proc. of 28th Int. Cosmic Ray Conf., Tsukuba 4 (2003) 183.

[451] T. Kifune et al. (Akeno Collab.), J. Phys. G: Nucl. Part. Phys. 12 (1986) 129.

[452] P. Gerhardy and R. Clay, J. Phys. G: Nucl. Part. Phys. 9 (1983) 1279.

[453] M. I. Pravdin, A. A. Ivanov, A. D. Krasilnikov, A. A. Mikhailov, and I. E. Sleptsov, J. Exp. Theor. Phys. 92 (2001) 766-770.

[454] N. Hayashida et al. (AGASA Collab.), Astropart. Phys. 10 (1999) 303-311

[455] J. A. Bellido, R. W. Clay, B. R. Dawson, and M. Johnston-Hollitt, Astropart. Phys. 15 (2001) $167-175$

[456] M. Aglietta et al. (Pierre Auger Collab.), Astropart. Phys. 27 (2007) 244-253

[457] R. Abbasi et al. (HiRes Collab.), Astropart. Phys. 21 (2004) 111

[458] J. D. Swain, Proc. of 10th Marcel Grossmann Meeting on Recent Developments in Theoretical and Experimental General Relativity, Gravitation and Relativistic Field Theories (MG X MMIII), Rio de Janeiro (2004) 721-740 and astro-ph/0401632.

[459] E. Armengaud et al. (Pierre Auger Collab.), Proc. of 30th Int. Cosmic Ray Conf., Merida 4 (2007) 175 and arXiv:0706.2640 [astro-ph].

[460] N. Hayashida et al. (AGASA Collab.), Phys. Rev. Lett. 77 (1996) 1000-1003.

[461] M. Takeda et al. (AGASA Collab.), Astrophys. J. 522 (1999) 225-237

[462] M. Teshima et al. (AGASA Collab.), Proc. of 28th Int. Cosmic Ray Conf., Tsukuba (2003) 437.

[463] R. U. Abbasi et al. (HiRes Collab.), Astropart. Phys. 22 (2004) 139-149

[464] R. U. Abbasi et al. (HiRes Collab.), Astrophys. J. 610 (2004) L73

[465] R. U. Abbasi et al. (HiRes Collab.), Astrophys. J. 623 (2005) 164-170

[466] S. Mollerach (Pierre Auger Collab.), Proc. of 30th Int. Cosmic Ray Conf., Merida 4 (2007) 279 and arXiv:0706.1749 [astro-ph].

[467] Y. Uchihori et al., Astropart. Phys. 13 (2000) 151-160

[468] E. E. Antonov et al., Izv. RAN, Ser. Fiz. 63 (1999) 542.

[469] A. V. Glushkov, Phys. Atom. Nucl. 66 (2003) 1252-1259.

[470] A. V. Glushkov and M. I. Pravdin, Phys. Atom. Nucl. 66 (2003) 854-863. 
[471] M. Kachelrieß and D. V. Semikoz, Astropart. Phys. 26 (2006) 10-15

[472] B. Revenu et al. (Pierre Auger Collab.), Proc. of 29th Int. Cosmic Ray Conf., Pune (2005) 75

[473] P. G. Tinyakov and I. I. Tkachev, JETP Lett. 74 (2001) 1-5

[474] P. G. Tinyakov and I. I. Tkachev, JETP Lett. 74 (2001) 445-448

[475] D. S. Gorbunov, P. G. Tinyakov, I. I. Tkachev, and S. V. Troitsky, Astrophys. J. 577 (2002) L93

[476] N. W. Evans, F. Ferrer, and S. Sarkar, Phys. Rev. D67 (2003) 103005

[477] P. Tinyakov and I. Tkachev, Phys. Rev. D69 (2004) 128301

[478] N. W. Evans, F. Ferrer, and S. Sarkar, Phys. Rev. D69 (2004) 128302

[479] B. E. Stern and J. Poutanen, Astrophys. J. 623 (2005) L33-L36

[480] R. U. Abbasi et al. (HiRes Collab.), Astrophys. J. 636 (2006) 680-684

[481] M. Veron-Cetty and P. Veron, Astron. Astrophys. 455 (2006) 773.

[482] D. S. Gorbunov, P. G. Tinyakov, I. I. Tkachev, and S. V. Troitsky, JETP Lett. 80 (2004) 145-148

[483] D. Harari (Pierre Auger Collab.), Proc. of 30th Int. Cosmic Ray Conf., Merida 4 (2007) 283 and arXiv:0706.1715 [astro-ph].

[484] J. Abraham et al. (Pierre Auger Collab.), Science 318 (2007) 939-943

[485] J. Abraham et al. (Pierre Auger Collab.), Astropart. Phys. 29 (2008) 188

[486] T. Stanev, arXiv:0805.1746 [astro-ph].

[487] T. Kashti and E. Waxman, JCAP 0805 (2008) 006

[488] G. Ghisellini, G. Ghirlanda, F. Tavecchio, F. Fraternali, and G. Pareschi, arXiv:0806.2393 [astro$\mathrm{ph}]$.

[489] H. Takami, T. Nishimichi, K. Yahata, and K. Sato, arXiv:0812.0424 [astro-ph].

[490] A. A. Ivanov and f. t. Y. a. group, Pisma Zh. Eksp. Teor. Fiz. 87 (2008) 215

[491] E. Berezhko and L. Ksenofontov, JETP 89 (1999) 391.

[492] K. Kobayakawa et al., Phys. Rev. D 66 (2002) 083004.

[493] T. Stanev, P. L. Biermann, and T. K. Gaisser, Astron. Astroph. 274 (1993) 902

[494] P. L. Biermann, N. Langer, E. Seo, and T. Stanev, Astron. \& Astroph. 369 (2001) .

[495] L. Sveshnikova et al., Astron. Astroph. 409 (2003) 799.

[496] R. Plaga, New Astronomy 7 (2002) 317.

[497] S. Wick et al., Astropart. Phys. 21 (2004) 125.

[498] A. Dar, Nuovo Cim. B120 (2005) 767-781

[499] A. Erlykin and A. Wolfendale, J. Phys. G: Nucl. Part. Phys. 27 (2001) 1005.

[500] H. Völk and V. Zirakashvili, Proc. of 28th Int. Cosmic Ray Conf., Tsukuba 4 (2003) 2031.

[501] N. Kalmykov and A. Pavlov, Proc. of 26th Int. Cosmic Ray Conf., Salt Lake City 4 (1999) 263.

[502] S. Ogio and F. Kakimoto, Proc. of 28th Int. Cosmic Ray Conf., Tsukuba 1 (2003) 315.

[503] E. Roulet, Int. J. Mod. Phys. A 19 (2004) 1133.

[504] A. Lagutin et al., Nucl. Phys. B (Proc. Suppl.) 97 (2001) 267.

[505] S. Swordy, Proc. of 24th Int. Cosmic Ray Conf., Rome 2 (1995) 697.

[506] S. Karakula and W. Tkaczyk, Astropart. Phys. 1 (1993) 229.

[507] M. Dova et al., preprint astro-ph/0112191 2001. 
[508] A. Dar and A. De Rujula, Phys. Rep. 466 (2006) 179-241

[509] V. Ptuskin et al., Astron. Astroph. 268 (1993) 726.

[510] R. Wigmans, Astropart. Phys. 19 (2003) 379.

[511] J. Candia et al., Astropart. Phys. 17 (2002) 23.

[512] S. Hannestad, New Journal of Physics 6 (2004) 108.

[513] D. Kazanas and A. Nikolaidis, Gen. Rel. Grav. 35 (2001) 1117.

[514] A. Petrukhin, Phys. Atom. Ncl. 66 (2003) 517.

[515] R. Wischnewski et al., Int. J. Mod. Phys. A 20 (2005) 6392.

[516] V. Ptuskin, Adv. Space Res. 19 (1997) 697.

[517] J. Candia, S. Mollerach, and E. Roulet, JCAP 0305 (2003) 003

[518] F. Aharonian et al. (H.E.S.S. Collab.), Nature 432 (2004) 75.

[519] H. Völk and E. Berezhko, Astron. Astroph. 451 (2006) 981.

[520] F. Aharonian et al. (H.E.S.S. Collab.), Astron. Astroph. 449 (2006) 223.

[521] E. Berezhko and H. Völk, arXiv:0810.0988 2008.

[522] F. Aharonian et al., Astron. Astroph. 370 (2001) 112.

[523] E. Berezhko et al., Astron. Astroph. 400 (2003) 971.

[524] H. J. Völk, Proc. of 7th Workshop on Towards a Network of Atmospheric Cherenkov Detectors 2005, Palaiseau (2006) and astro-ph/0603502

[525] A. M. Hillas, J. Phys. G31 (2005) R95-R131.

[526] T. Wibig and A. Wolfendale, J. Phys. G: Nucl. Part. Phys. 31 (2005) 255.

[527] V. S. Berezinsky, S. I. Grigorieva, and B. I. Hnatyk, Astropart. Phys. 21 (2004) 617-625

[528] V. Berezinsky, A. Z. Gazizov, and S. I. Grigorieva, Phys. Lett. B612 (2005) 147-153

[529] T. Wibig and A. W. Wolfendale, J. Phys. G31 (2005) 255-264

[530] D. Hooper, S. Sarkar, and A. M. Taylor, Astropart. Phys. 27 (2007) 199-212

[531] H. Takami, K. Murase, S. Nagataki, and K. Sato, arXiv:0704.0979 [astro-ph].

[532] M. Ahlers, L. A. Anchordoqui, and S. Sarkar, arXiv:0902.3993 [astro-ph.HE].

[533] A. A. Watson, Proc. of Conf. on Thinking, Observing and Mining the Universe, Sorrento, Italy (2003)

[534] D. Allard, N. G. Busca, G. Decerprit, A. V. Olinto, and E. Parizot, JCAP 0810 (2008) 033

[535] D. Hooper, A. Taylor, and S. Sarkar, Astropart. Phys. 23 (2005) 11-17

[536] L. A. Anchordoqui, H. Goldberg, D. Hooper, S. Sarkar, and A. M. Taylor, Phys. Rev. D76 (2007) 123008

[537] N. G. Lehtinen, P. W. Gorham, A. R. Jacobson, and R. A. Roussel-Dupre, Phys. Rev. D69 (2004) 013008

[538] A. W. Strong, I. V. Moskalenko, and O. Reimer, Astrophys. J. 613 (2004) 956-961

[539] S. W. Barwick et al. (ANITA Collab.), Phys. Rev. Lett. 96 (2006) 171101

[540] T. Stanev, P. L. Biermann, J. Lloyd-Evans, J. P. Rachen, and A. Watson, Phys. Rev. Lett. 75 (1995) 3056-3059

[541] I. Zaw, G. R. Farrar, and J. E. Greene, arXiv:0806.3470 [astro-ph].

[542] T. Wibig and A. W. Wolfendale, arXiv:0712.3403 [astro-ph]. 
[543] M. Kachelrieß, Nucl. Phys. Proc. Suppl. 165 (2007) 272-279

[544] M. Kachelrieß and P. D. Serpico, Phys. Lett. B640 (2006) 225-229

[545] M. Kachelrieß, P. D. Serpico, and M. Teshima, Astropart. Phys. 26 (2006) 378-386

[546] T. Antoni et al. (KASCADE Collab.), J. Phys. G: Nucl. Part. Phys. 27 (2001) 1785.

[547] A. Haungs et al. (KASCADE Collab.), Nucl. Phys. Proc. Suppl. 122 (2003) 384-387.

[548] T. Antoni et al. (KASCADE Collab.), Astropart. Phys. 19 (2003) 703.

[549] J. Hörandel et al. (KASCADE Collab.), Nucl. Phys. B (Proc. Suppl.) 122 (2003) 309.

[550] T. Antoni et al. (KASCADE Collab.), Phys. Rev. D 71 (2005) 072002.

[551] W. D. Apel et al. (KASCADE Collab.), J. Phys. G34 (2007) 2581-2593.

[552] W. D. Apel et al. (KASCADE-Grande Collab.), J. Phys. G36 (2009) 035201

[553] J. Milke et al. (KASCADE Collab.), Acta Physica Polonica B 35 (2004) 341.

[554] J. Milke et al. (KASCADE Collab.), Proc. of 29th Int. Cosmic Ray Conf., Pune 6 (2005) 125.

[555] J. Hörandel et al. preprint astro-ph/0509253 2005.

[556] CMS Collaboration, J. Instr. 3 (2008) S08004.

[557] L. Evans et al., J. Instr. 3 (2008) S08001.

[558] A. Haungs, L. W. Jones, and H. Rebel, NEEDS Workshop, Karlsruhe April 18 - 20, 2002, http://www-ik.fzk.de/ needs/, 2002.

[559] R. Engel, Nucl. Phys. Proc. Suppl. 122 (2003) 437-446

[560] R. Engel, Nucl. Phys. Proc. Suppl. 122 (2003) 40-55.

[561] A. Chiavassa et al. (KASCADE Collab.), Proc. of 29th Int. Cosmic Ray Conf., Pune 6 (2005) 313.

[562] M. Kestel et al., Nucl. Instrum. Meth. A 535 (2004) 139.

[563] H. Klages et al. (Pierre Auger Collab.), Proc. of 30th Int. Cosmic Ray Conf., Merida 5 (2007) 849.

[564] M. C. Medina et al., Nucl. Instrum. Meth. A566 (2006) 302-311

[565] C. C. H. Jui (TA Collab.), Proc. of 29th Int. Cosmic Ray Conf., Pune 8 (2005) 327.

[566] D. Nitz et al. (Pierre Auger Collab.), Proc of 30th Int. Cosmic Ray Conf., Merida 5 (2007) 889

[567] Y. Takahashi et al. (EUSO Collab.), Proc. of 30th Int. Cosmic Ray Conf., Merida 5 (2007) 1145.

[568] http://www.euso-mission.org/.

[569] M. Israel et al., ACCESS: A Cosmic Journey (Formulation Study Report of the NASA ACCESS Working Group) 2000. 